\title{
THE HERSCHEL INVENTORY OF THE AGENTS OF GALAXY EVOLUTION IN THE MAGELLANIC CLOUDS, A HERSCHEL OPEN TIME KEY PROGRAM
}

\author{
M. Meixner ${ }^{1,2}$, P. Panuzzo $^{3,4}$, J. Roman-Duval $^{1}$, C. Engelbracht ${ }^{5,6}$, B. Babler $^{7}$, J. Seale $^{1}$, S. Hony $^{3}$, E. Montiel $^{5,8}$, \\ M. Sauvage ${ }^{3}$, K. Gordon ${ }^{1}$, K. Misselt ${ }^{5}$, K. Okumura ${ }^{3}$, P. Chanial $^{3}$, T. Beck ${ }^{1}$, J.-P. Bernard ${ }^{9}, 10$, A. Bolatto $^{11}$, C. Bot $^{12,13}$,

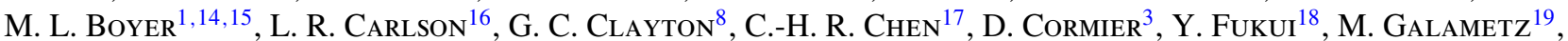 \\ F. Galliano ${ }^{3}$, J. L. Hora ${ }^{20}$, A. Hughes ${ }^{21}$, R. Indebetouw ${ }^{22}$, F. P. IsRael ${ }^{16}$, A. KAWAMura ${ }^{23}$, F. KemPeR ${ }^{24}$, S. Kim $^{25}$, \\ E. $\mathrm{Kwon}^{25}$, V. Lebouteiller ${ }^{3}$, A. Li $^{26}$, K. S. Long ${ }^{1}$, S. C. Madden ${ }^{3}$, M. Matsuura ${ }^{27}$, E. MulleR ${ }^{23}$, J. M. Oliveira ${ }^{28}$, \\ T. Onishi ${ }^{29}$, M. Otsuka ${ }^{24}$, D. Paradis ${ }^{9,10}$, A. Poglitsch ${ }^{30}$, W. T. Reach ${ }^{31}$, T. P. Robitaille ${ }^{21}$, M. Rubio $^{32}$, B. Sargent $^{1,33}$, \\ M. SewiŁo ${ }^{2}$, R. Skibba ${ }^{5}$, L. J. Smith ${ }^{34}$, S. Srinivasan ${ }^{24,35}$, A. G. G. M. Tielens ${ }^{16}$, J. Th. Van Loon ${ }^{28}$, And B. Whitney ${ }^{7}$ \\ ${ }^{1}$ Space Telescope Science Institute, 3700 San Martin Drive, Baltimore, MD 21218, USA; meixner@ stsci.edu \\ ${ }^{2}$ Department of Physics and Astronomy, The Johns Hopkins University, 366 Bloomberg Center, 3400 North Charles Street, Baltimore, MD 21218, USA \\ ${ }^{3}$ CEA, Laboratoire AIM, Irfu/SAp, Orme des Merisiers, F-91191 Gif-sur-Yvette, France \\ ${ }^{4}$ CNRS, Observatoire de Paris-Lab. GEPI, Bat. 11, 5, Place Jules Janssen, F-92195 Meudon Cedex, France \\ ${ }^{5}$ Steward Observatory, University of Arizona, 933 North Cherry Avenue, Tucson, AZ 85721, USA \\ ${ }^{6}$ Raytheon Company, 1151 East Hermans Road, Tucson, AZ 85756, USA \\ ${ }^{7}$ Department of Astronomy, 475 North Charter Street, University of Wisconsin, Madison, WI 53706, USA \\ ${ }^{8}$ Department of Physics and Astronomy, Louisiana State University, 233-A Nicholson Hall, Tower Drive, Baton Rouge, LA 70803-4001, USA \\ ${ }^{9}$ CNRS, IRAP, 9 Av. Colonel Roche, BP 44346, F-31028 Toulouse Cedex 4, France \\ ${ }^{10}$ Université de Toulouse, UPS-OMP, IRAP, F-31028 Toulouse Cedex 4, France \\ ${ }^{11}$ Department of Astronomy, Laboratory for Millimeter-Wave Astronomy, University of Maryland, College Park, MD 20742-2421, USA \\ ${ }^{12}$ Université de Strasbourg, Observatoire Astronomique de Strasbourg, 11, Rue de l'Universite, F-67000 Strasbourg, France \\ ${ }^{13}$ CNRS, Observatoire Astronomique de Strasbourg, UMR7550, F-67000 Strasbourg, France \\ ${ }^{14}$ Observational Cosmology Laboratory, Code 665, NASA Goddard Space Flight Center, Greenbelt, MD 20771, USA \\ ${ }^{15}$ Oak Ridge Associated Universities (ORAU), Oak Ridge, TN 37831, USA \\ ${ }^{16}$ Sterrewacht Leiden, Leiden University, P.O. Box 9513, NL-2300 RA Leiden, The Netherlands \\ ${ }_{17}$ Max-Planck-Institut für Radioastronomie Auf dem Hügel 69, D-53121 Bonn, Germany \\ ${ }^{18}$ Department of Astrophysics, Nagoya University, Chikusa-ku, Nagoya 464-8602, Japan \\ ${ }^{19}$ Institute of Astronomy, University of Cambridge, Madingley Road, Cambridge CB3 0HA, UK \\ ${ }^{20}$ Center for Astrophysics, 60 Garden Street, MS 67, Harvard University, Cambridge, MA 02138, USA \\ ${ }^{21}$ Max-Planck-Institut für Astronomie, Königstuhl 17, D-69117 Heidelberg, Germany \\ 22 Department of Astronomy, University of Virginia, and National Radio Astronomy Observatory, 520 Edgemont Road, Charlottesville, VA 22903, USA \\ ${ }^{23}$ National Astronomical Observatory of Japan, 2-21-1, Osawa, Mitaka, Tokyo 181-8588, Japan \\ ${ }^{24}$ Academia Sinica, Institute of Astronomy and Astrophysics, P.O. Box 23-141, Taipei 10617, Taiwan \\ ${ }^{25}$ Department of Astronomy and Space Science, Sejong University, 98 Gunja-dong, Gwangjin-gu, 143-747 Seoul, Korea \\ ${ }^{26}$ Department of Physics and Astronomy, 314 Physics Building, University of Missouri, Columbia, MO 65211, USA \\ ${ }^{27}$ Department of Physics and Astronomy, University College London, Gower Street, London WC1E 6BT, UK \\ ${ }^{28}$ School of Physical and Geographical Sciences, Lennard-Jones Laboratories, Keele University, Staffordshire ST5 5BG, UK \\ 29 Department of Physical Science, Osaka Prefecture University, Gakuen 1-1, Sakai, Osaka 599-8531, Japan \\ ${ }^{30}$ Max-Planck-Institut für extraterrestrische Physik, Giessenbachstraße, D-85748 Garching, Germany \\ ${ }^{31}$ Stratospheric Observatory for Infrared Astronomy, Universities Space Research Association, Mail Stop 232-11, Moffett Field, CA 94035, USA \\ 32 Departamento de Astronomía, Universidad de Chile, Casilla 36-D, Santiago, Chile \\ ${ }^{33}$ Center for Imaging Science and Laboratory for Multiwavelength Astrophysics, Rochester Institute of Technology, \\ 54 Lomb Memorial Drive, Rochester, NY 14623, USA \\ ${ }^{34}$ Space Telescope Science Institute and European Space Agency, 3700 San Martin Drive, Baltimore, MD 21218, USA \\ ${ }^{35}$ UPMC-CNRS UMR7095, Institute d'Astrophysique de Paris, F-75014 Paris, France \\ Received 2012 November 27; accepted 2013 July 8; published 2013 August 9
}

\begin{abstract}
We present an overview of the HERschel Inventory of The Agents of Galaxy Evolution (HERITAGE) in the Magellanic Clouds project, which is a Herschel Space Observatory open time key program. We mapped the Large Magellanic Cloud (LMC) and Small Magellanic Cloud (SMC) at 100, 160, 250, 350, and $500 \mu \mathrm{m}$ with the Spectral and Photometric Imaging Receiver (SPIRE) and Photodetector Array Camera and Spectrometer (PACS) instruments on board Herschel using the SPIRE/PACS parallel mode. The overriding science goal of HERITAGE is to study the life cycle of matter as traced by dust in the LMC and SMC. The far-infrared and submillimeter emission is an effective tracer of the interstellar medium (ISM) dust, the most deeply embedded young stellar objects (YSOs), and the dust ejected by the most massive stars. We describe in detail the data processing, particularly for the PACS data, which required some custom steps because of the large angular extent of a single observational unit and overall the large amount of data to be processed as an ensemble. We report total global fluxes for the LMC and SMC and demonstrate their agreement with measurements by prior missions. The HERITAGE maps of the LMC and SMC are dominated by the ISM dust emission and bear most resemblance to the tracers of ISM gas rather than the stellar content of the galaxies. We describe the point source extraction processing and the criteria used to establish a catalog for each waveband for the HERITAGE program. The $250 \mu \mathrm{m}$ band is the most sensitive and the source catalogs for this band have $\sim 25,000$ objects for the LMC and $\sim 5500$ objects for the SMC. These data enable studies of ISM dust properties, submillimeter excess dust emission, dust-to-gas ratio, Class 0 YSO candidates, dusty massive evolved stars, supernova remnants (including SN1987A), H II regions, and dust evolution in the LMC and SMC. All images and catalogs are delivered to the Herschel Science Center as part of the community support aspects of
\end{abstract}


the project. These HERITAGE images and catalogs provide an excellent basis for future research and follow up with other facilities.

Key words: catalogs - dust, extinction - infrared: galaxies - Magellanic Clouds - submillimeter: general - surveys

Online-only material: color figures

\section{INTRODUCTION}

The Large Magellanic Cloud (LMC) and Small Magellanic Cloud (SMC) are excellent astrophysical laboratories for studying the life cycle of the interstellar medium (ISM). Located at 50 and $60 \mathrm{kpc}$, their proximity permits detailed studies of resolved ISM clouds and their relation to stellar populations on global scales. In the Milky Way (MW), which is difficult to study in its entirety, ISM studies are more challenging because they suffer from line-of-sight crowding and distance ambiguity that requires extensive work to disentangle (e.g., Reid et al. 2009). In the LMC and SMC, ISM clouds and stars are at a similar distance, rendering luminosities directly comparable. In particular, the thin-disk morphology and favorable viewing angle of the LMC make the comparison between dust emission and gas line emission fairly straightforward (Table 1). The Magellanic Bridge is a filament of neutral hydrogen, which joins the SMC and LMC over some $15 \mathrm{kpc}$ (Muller et al. 2003a; Hindman et al. 1963; Mathewson 1985; Staveley-Smith et al. 1998). The part of the Magellanic Bridge that is closest to the SMC has been called the Tail, a term that we adopt for this paper. The Tail has evidence of recent star formation (Harris 2007; Grondin et al. 1992; Hambly et al. 1994; Chen et al. 2013). When we refer to the mapping observations of the SMC, we are referring to the SMC+Tail region.

The LMC and SMC differ substantially in many global properties such as metallicity, molecular gas content, and gas-to-dust ratio thus allowing us to study how the processes governing galaxy evolution depend on these properties (Table 1). The physical processes leading to star-formation (in particular, dynamics, $\mathrm{H}-\mathrm{H}_{2}$ transition, gas cooling) can be examined at metallicities of 0.5 (LMC) and 0.2 (SMC; Table 1) which is similar to galaxies at the epoch of peak star formation in the universe (Madau et al. 1996; Pei et al. 1999). The lower metallicity results in lower dust content in both galaxies compared to the MW (Table 1). Less dust in the ISM increases the mean free path for stellar photons from the massive OB star population which in turn increases the ambient UV radiation fields to higher values than in the solar neighborhood. In terms of galaxy evolution, the LMC-SMC pair is well suited to studying how the agents of evolution, the ISM and stars, operate as a whole in two galaxies that are tidally interacting with each other and with the MW (e.g., Zaritsky \& Harris 2004; Bekki \& Chiba 2005; Besla et al. 2007; Gardiner \& Noguchi 1996).

Full surveys of the LMC and SMC provide both a detailed view of astrophysical processes occurring within these galaxies and an opportunity for statistical assessment of these processes over an entire galaxy. Moreover, a whole-galaxy survey provides a complete template for high-redshift galaxies. The SMC and LMC have been surveyed at many wavelengths revealing structures on all scales thereby providing a rich context for investigations. The stellar content has been photometrically surveyed at optical (MCPS; Zaritsky et al. 2002, 2004) and near-infrared (near-IR) wavelengths (Two Micron All Sky Survey (2MASS), Skrutskie et al. 2006, Nikolaev \& Weinberg 2000; Vista Magel- lanic Cloud Survey, Cioni et al. 2011) and for stellar variability (MACHO, Alcock et al. 2000; IRSF, Ita et al. 2004; OGLE III, Udalski et al. 2008a, 2008b). The ISM gas content has been traced in atomic hydrogen with the $\mathrm{HI}_{\mathrm{I}} 21 \mathrm{~cm}$ line (ATCA+Parkes, Kim et al. 2003; Parkes, Stanimirović et al. 1999, Muller et al. 2003a), in ionized gas content in the SHASSA survey by Gaustad et al. (2001), and in molecular gas content in the CO rotational transitions in the NANTEN, MAGMA, and mini-south Columbia surveys (Fukui et al. 2008; Wong et al. 2011; Mizuno et al. 2001; Cohen et al. 1988; Rubio et al. 1991) and spectroscopically for $\mathrm{H}_{\mathrm{I}}$ and $\mathrm{H}_{2}$ surveys (Tumlinson et al. 2002). Molecular and atomic gas fractions vary greatly between the LMC and SMC (Table 1). The dust content, relative to the gas, has been analyzed in UV spectra along lines of sight to bright stars (Gordon et al. 2003; Maíz Apellániz \& Rubio 2012). The emission from dust has been surveyed with IRAS (Schwering \& Israel 1989), and the Midcourse Space Experiment (Egan et al. 2001). The extinction by dust has been studied by Dobashi et al. (2008).

In our Spitzer Surveying the Agents of Galaxy Evolution (SAGE) surveys, we have invested $\gtrsim 1000 \mathrm{hr}$ fully surveying the LMC and SMC over the full wavelength range $(3.5-160 \mu \mathrm{m}$; Meixner et al. 2006; Gordon et al. 2011) with the Spitzer IRAC and MIPS cameras and with the Infrared Spectrograph for hundreds of point sources and for tens of targeted ISM regions to study the lifecycle of galaxies (Kemper et al. 2010). The Spitzer Survey of the SMC (Bolatto et al. 2007) pre-dated the SAGE surveys and covered the brightest regions of the SMC. These Spitzer studies were very successful in providing galaxywide overviews of the emission of warm dust in the ISM (e.g., Bernard et al. 2008), discovery of thousands of young stellar objects (YSOs; e.g., Whitney et al. 2008; Gruendl \& Chu 2009; Carlson et al. 2012; Sewiło et al. 2013), and a census of the mass injected by tens of thousands of dusty evolved stars (e.g., Srinivasan et al. 2009; Boyer et al. 2011, 2012; Riebel et al. 2012). The analysis of the dust emission in the ISM compared to the gas emission reveals higher dust-to-gas ratios than for the UV extinction measurements (Table 1) suggesting either missing gas mass as traced by $\mathrm{H}_{\mathrm{I}} 21 \mathrm{~cm}$ or $\mathrm{CO}$ or there are large variations in the dust-to-gas ratio.

In this paper, we present an overview of the HERschel Inventory of The Agents of Galaxy Evolution (HERITAGE) program, an open time key program on the Herschel Space Observatory (Pilbratt et al. 2010). HERITAGE provides an imagingphotometric survey with the Photodetector Array Camera and Spectrometer (PACS; Poglitsch et al. 2010) and Spectral and Photometric Imaging Receiver (SPIRE; Griffin et al. 2010) at $100,160,250,350$, and $500 \mu \mathrm{m}$. These crucial long wavelengths complement the Spitzer Space Telescope studies of the LMC and SMC. We discuss the observing strategy in Section 2, while the data processing steps are described in Sections 3 and 4. Sections 5 and 6 describe the spatial distribution of the imaged emission and the Magellanic Clouds' total integrated Herschel emission, respectively. The source catalog creation is described in Section 7, and the makeup of the catalog is discussed in 
Table 1

Properties of the Large Magellanic Cloud and Small Magellanic Cloud

\begin{tabular}{lccc}
\hline \hline Physical Property & LMC & SMC+Tail & References \\
\hline Distance $(\mathrm{kpc})$ & 50 & 61 & 1,2 \\
Disk thickness $(\mathrm{kpc})$ & 2.5 & 4.2 & 3 \\
Inclination & $23^{\circ}-37^{\circ}$ & $62^{\circ}$ & 3 \\
Metallicity $\left(Z_{\odot}\right)$ & 0.5 & $0.1-0.2$ & 4,5 \\
ISM gas size $(\mathrm{kpc})$ & 8 & 3 & $6,7,8$ \\
H I gas mass $\left(M_{\odot}\right)$ & $4.8 \times 10^{8}$ & $4.2 \times 10^{8}$ & $9,7,8$ \\
CO gas mass $\left(M_{\odot}\right)$ & $4 \times 10^{7}$ & $4 \times 10^{6}$ & $10,11,12$ \\
Dust-to-gas ratio $(\mathrm{UV})$ & 0.005 & 0.001 & 13 \\
Dust-to-gas ratio $(\mathrm{IR} /$ radio $)$ & 0.0017 & $0.00011-0.00083$ & $14,15,16$ \\
Stellar mass $\left(M_{\odot}\right)$ & $1.7 \times 10^{9}$ & $3.7 \times 10^{8}$ & 17,18 \\
Star formation rate $\left(M_{\odot} \mathrm{yr}^{-1}\right)$ & $0.1-0.25$ & $0.024-0.05$ & $17,19,20,21,22$ \\
\hline
\end{tabular}

References. (1) Ngeow \& Kanbur 2008; (2) Szewczyk et al. 2009; (3) Subramanian \& Subramaniam 2009, 2010 , 2012; (4) Russell \& Dopita 1992, corrected by Asplund et al. 2004; (5) Lee et al. 2005; (6) Kim et al. 1998; (7) Stanimirović et al. 1999; (8) Muller et al. 2003a; (9) Staveley-Smith et al. 2003; (10) Fukui et al. 2008; (11) Mizuno et al. 2001; (12) Muller et al. 2003b; (13) Gordon et al. 2003; (14) Bernard et al. 2008; (15) Bot et al. 2004; (16) Gordon et al. 2009; (17) Skibba et al. 2012; (18) Harris \& Zaritsky 2004, 2009; (19) Whitney et al. 2008; (20) Sewiło et al. 2013; (21) Bolatto et al. 2011; (22) Wilke et al. 2004.

Table 2

Principal Characteristics of the HERITAGE Survey, Herschel Program ID KPOT_mmeixner_1

\begin{tabular}{|c|c|c|}
\hline Parameter & LMC & SMC \\
\hline R.A. (J2000) & $05^{\mathrm{h}} 18^{\mathrm{m}} 48^{\mathrm{s}}$ & $01^{\mathrm{h}} 30^{\mathrm{m}} 16^{\mathrm{s}} .8$ \\
\hline Decl. (J2000) & $-68^{\circ} 34^{\prime} 12^{\prime \prime}$ & $-73^{\circ} 22^{\prime} 58^{\prime \prime} .8$ \\
\hline Areal coverage & $8^{\circ} \times 8.5$ & $5^{\circ} \times 5^{\circ}+4^{\circ} \times 3^{\circ}$ \\
\hline AOR sizes & $30^{\prime}-36^{\prime} \times 450^{\prime}-495^{\prime}$ & $80^{\prime}-102^{\prime} \times 120^{\prime}-240^{\prime}$ \\
\hline Epoch 1 dates & 2010 Apr 28 to May 2 & 2010 Mar 24-25 \\
\hline Epoch 1 OBSID ${ }^{a}$ & $1342195668,1342195669,1342195683$ & 1342192680, 1342192681, 1342192697, \\
\hline$\cdots$ & $1342195684,1342195707,1342195708$ & 1342192698,1342192699 \\
\hline$\ldots$ & $1342195712,1342195713,1342195728$ & $\ldots$ \\
\hline Epoch 2 dates & 2010 Aug $2-7$ & 2010 Jun $18-21$ \\
\hline Epoch 2 OBSID & $1342202086,1342202087,1342202202$ & $1342198565,1342198566,1342198590$, \\
\hline$\cdots$ & $1342202203,1342202216,1342202217$ & 1342198591,1342198863 \\
\hline$\cdots$ & $1342202224,1342202225,1342202243$ & $\cdots$ \\
\hline$\ldots$ & 1342202244 & $\cdots$ \\
\hline Epoch $3^{b}$ dates & & 2010 Sep $23-24$ \\
\hline Epoch 3 OBSID & & $\begin{array}{c}1342205049,1342205050, \\
1342205054,1342205055,1342205092\end{array}$ \\
\hline
\end{tabular}

Notes.

a OBSID is the reference number for the AOR executed and can be used to identify the data in the Herschel science archive (HSA). For epoch 1, we note that for the SMC, these OBSID only include the PACS data (see text).

b We have an epoch 3 data set for the SMC only because it was a makeup for the epoch 1. Epoch 3 has both PACS and SPIRE data.

Section 8. We conclude with a description of the higher level data products available to the community in Section 9 and a summary in Section 10.

\section{OBSERVATIONAL PLAN}

Using $285 \mathrm{hr}$ of Herschel observations, we have performed a uniform photometric survey of the LMC $\left(8^{\circ} \times 8.5\right)$, SMC $\left(5^{\circ} \times 5^{\circ}\right.$ and $\left.4^{\circ} \times 3^{\circ}\right)$ in all SPIRE bands $(250,350,500 \mu \mathrm{m})$ and in the 100 and $160 \mu \mathrm{m}$ PACS bands. The raw data observations have been stored in the Herschel archive under proposal KPOT_mmeixner_1 where KPOT stands for "key program in open time." The map sizes for the LMC and SMC were chosen to fully encompass each galaxy as observed at $160 \mu \mathrm{m}$ (Spitzer/MIPS, Figure 1). Observing characteristics for the survey have been outlined in Table 2.

For the full SPIRE/PACS imaging of the LMC and SMC, our science goals were primarily driven by the need to spatially map the coldest $(T<15 \mathrm{~K})$ dust, which requires SPIRE. The ability to acquire high-quality PACS images at 100 and $160 \mu \mathrm{m}$ for a minimum of extra time using the SPIRE/PACS parallel mode fulfilled secondary science drivers getting better wavelength coverage $(100 \mu \mathrm{m})$ and spatial resolution $(160 \mu \mathrm{m})$ of the large, warm $(25 \mathrm{~K}<T<15 \mathrm{~K})$ grains and compact source spectral energy distributions (SEDs) than prior observations by IRAS and Spitzer/MIPS. We efficiently and fully mapped the LMC and SMC at 100, 160, 250, 350, and $500 \mu \mathrm{m}$ using the SPIRE/PACS parallel mode at low scanning speed $\left(20^{\prime \prime} \mathrm{s}^{-1}\right)$. The low scanning speed mode was picked as it allowed us to reach our sensitivity goals, to distinguish between glitches and sources in the timeline, and to produce minimally smeared PACS point spread functions (PSFs).

The astronomical observing requests (AORs), ranging from a few to $9 \mathrm{hr}$ in duration, were designed to scan over each galaxy in long strips to ensure that background measurements were made 

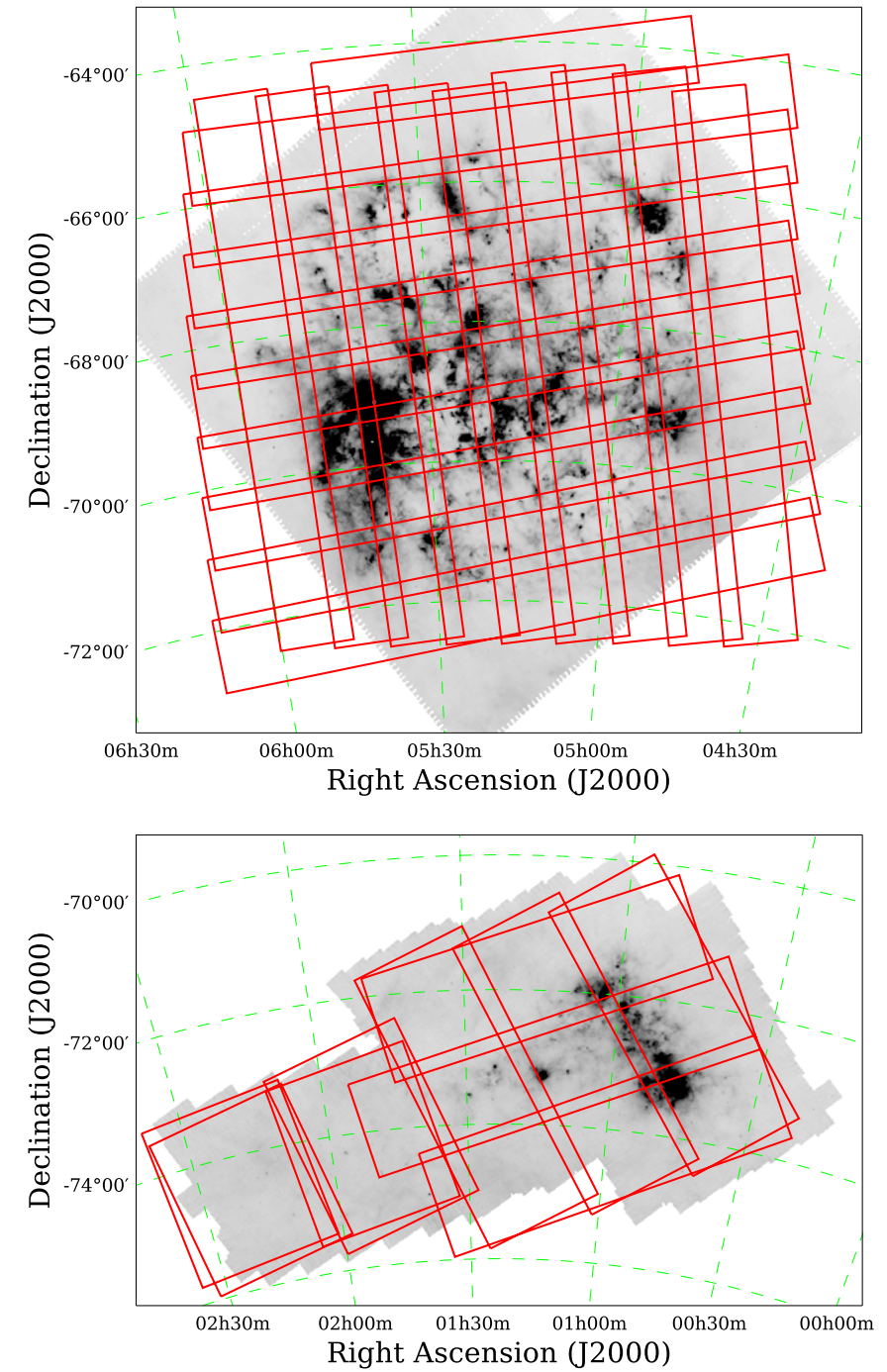

Figure 1. HERITAGE SPIRE AOR mapping strategy for the LMC (top) and SMC (bottom) overlaid on the MIPS $160 \mu \mathrm{m}$ image from the SAGE-LMC Spitzer Legacy program (Meixner et al. 2006) and SAGE-SMC Spitzer Legacy program (Gordon et al. 2011). Each long red rectangle represents the footprint of one AOR (see Table 2 for details on sizes). The long scans of each epoch are sufficient to get off the main galaxy far-infrared emissions and sample a suitable background. The AORs for both epoch 1 and epoch 2 are shown. Because the epochs were separated in time by approximately 3 months, the AORs are orthogonal and the combination of both epoch footprints results in a basketweave appearance. This observing strategy improved the final photometry quality of the combined-epoch maps.

(A color version of this figure is available in the online journal.)

at the beginning and end of each scan leg, allowing removal of the $1 / f$ noise with timescales longer than the time to take a single scan leg. To further suppress the $1 / f$ noise, each map was repeated with the scan angle rotated $90^{\circ}$, which naturally happened in about 3 months at the positions of the LMC and SMC. To effect this orthogonal rotation in the mapping we scheduled the observations to have a three-month separation between epochs 1 and 2 . This observing strategy is similar to the strategy that we adopted for the MIPS observations on the SAGE-LMC (Meixner et al. 2006) and SAGE-SMC (Gordon et al. 2011) projects. However, we note that it differs from other large-area mapping programs with Herschel, e.g., HIGAL (Molinari et al. 2010), which used smaller AOR tiles and the HSPOT "orthogonal" option to obtain a perpendicular direction as part of the AOR. Our observing strategy had some unforeseen consequences for the PACS data processing within the Herschel Interactive Processing Environment (HIPE) which we discuss below.

The scan angle rotates by $1^{\circ} \mathrm{day}^{-1}$ for the Magellanic Clouds, so we concatenated several AORs in order to make a complete map without gaps in the minimum time at each epoch. For the LMC, the epoch 1 date range was 2010 April 28 to May 2 and the epoch 2 date range was 2010 August 2-7 (Figure 1). For the SMC, the epoch 1 date range was 2010 March 24 and 25; however, although the PACS data were successfully obtained, the SPIRE observations failed due to commanding issues. Thus for the SMC, we rescheduled observations with epoch 2 date range of 2010 June 18-21 and epoch 3 date range of 2010 September 23 and 24. Although the LMC mapped region is approximately square in shape, we used non-uniform sized strips to cover the LMC in an optimal fashion (Figure 1 and Table 2) in order to meet our time constraints. The rectangular SMC coverage was broken into two approximately square shaped regions for the mapping the SMC and Tail regions (Figure 1 and Table 2).

\section{PACS DATA PROCESSING}

\subsection{Background}

The HERITAGE data were obtained in scan mapping mode. Scan maps are performed by slewing the spacecraft at a constant speed along parallel lines (see chapter 5.1 of the PACS observer's manual). In the following, we refer to a scan leg as one of these scan lines, i.e., a continuous segment of data acquired between turnarounds. During each AOR, the arrays of PACS bolometers $(32 \times 16$ bolometers for the $160 \mu \mathrm{m}$ band and $64 \times 32$ for the $100 \mu \mathrm{m}$ band) sample the far-infrared (FIR) dust emission as a function of time as the spacecraft performs the scans. As a result, raw PACS data consist of a three-dimensional cube, the first two dimensions corresponding to the spatial dimensions of the array, and the third dimension to time, which can be directly transformed into position on the sky using the pointing information. We refer to a frame as the data in the PACS bolometer array at time $t$ (a frame is therefore two-dimensional, 2D), and to a timeline as the data recorded in one bolometer as a function of time (a timeline is onedimensional, 1D). At the end of the data processing, the timelines therefore need to be projected onto the sky, leading to the creation of a 2D map of the FIR emission recorded by PACS.

The PACS bolometers are affected by the so-called $1 / f$ noise caused by thermal drifts where the amplitude of the noise varied inversely with frequency. Measurements of the PACS low frequency noise indicated it approximately followed a $1 / \sqrt{f}$ relation (Sauvage \& Okumura 2009). In addition, the HERITAGE AORs were the longest in angular extent for the Herschel mission, with an extent of about $\sim 8^{\circ}$ in length for the $\mathrm{LMC}$, and $\sim 5^{\circ}$ for the SMC. As a result, the HERITAGE PACS timelines are dominated by baseline drifts resulting from $1 / f$ noise, which therefore need to be mitigated prior to projection of the data onto the sky. In addition to these drifts, cosmic ray hits on bolometers and readout electronics cause glitches or sudden jumps in the signal strength of the timeline. These glitches also need to be removed during the data processing.

Noise, drifts, and glitches can be estimated and removed using the redundancy of the observations, since each region of the sky is seen by several bolometers during the scans and cross-scans. Software programs based on the redundancy are available to 
mitigate drifts and $1 / f$ noise, such as MADmap (Cantalupo et al. 2010), Scanamorphos (Roussel 2012) and Tamasis (P. Chanial et al., in preparation). Because they are based on redundancy, these algorithms require PACS data to be processed in its entirety in one run. In order to use such algorithms on the large amount of data collected by the HERITAGE survey would have required substantial computing resources, perhaps a supercomputer. For example the LMC PACS $100 \mu \mathrm{m}$ data takes 200 Gbyte, which was well beyond our 128 Gbyte RAM server. For a future data release, we will work with the Tamasis program on a supercomputer to generate PACS images of the LMC and SMC. However, for the initial data release we have processed the data using our own custom pipeline that included some steps in addition to the standard PACS data processing software in order to appropriately remove the baseline. Our custom approach fits within the computational resources of our 128 Gbyte RAM server. Our initial approach creates images which have residual $1 / f$ noise that limits the scientific utility of the data for some projects, e.g., power-spectral analysis of the ISM structure. The different steps of this custom algorithm are described in the following subsections. Figure 2 illustrates the steps of the PACS data processing, especially the baseline subtraction, for one timeline corresponding to one bolometer.

\subsection{Basic Reduction from Level 0 to Level 1}

The basic PACS data reduction was performed starting from the level 0 product to the level 1 product using the standard pipeline software in the HIPE version 7.0 data reduction software (Ott 2010; PACS data reduction guide ${ }^{36}$ ). First, calibration blocks are identified in the telemetry and processed. Bad and saturated pixels are masked. Readouts are converted into voltages. Pointing information and sky coordinates are added to the frames. The signal (in volts) is converted to Jy using the responsivity factors in HIPE 7.0, and a flat field correction is applied. After these steps, we exported the data as FITS files for further processing within the IDL software environment because the HIPE pipeline software was unable to accomplish the task.

\subsection{First-order Baseline Subtraction}

The level 1 frames in the timeline data were compromised by $1 / f$ noise which was caused by drifts in the bolometer signal with time. There were also large pixel-to-pixel offsets between bolometers. This made the removal of glitches, which were sudden jumps in the timelines due to cosmic rays hitting the detectors, very inefficient with the algorithms implemented in HIPE. As a result, a first-order, rough baseline subtraction was implemented (in IDL) before applying the deglitching algorithm. For this purpose, we split the signal timeline of each bolometer by scan leg. For each scan leg and each bolometer, we estimated the median signal at the end points of the scan, located outside of the LMC or SMC, where we assumed that the FIR flux was zero. The median of the signal at the end points was estimated in a 600 frame long $\left(10^{\prime}\right)$ window for the PACS $160 \mu \mathrm{m}$ band, and 300 frame long $\left(10^{\prime}\right)$ window for the PACS $100 \mu \mathrm{m}$ band. Regions of the timelines located within 1000 frames of a calibration block were masked out to eliminate the influence of transients, which were drifts in the signal following a calibration block. A linear fit to the median values of the timelines at the scan ends was then estimated, and subtracted from the timeline corresponding to the scan leg of

$\overline{36}$ The PACS user guide is on line at http://herschel.esac.esa.int/hcss-doc-7.0/. each bolometer. This process is illustrated in the top two panels of Figure 2, where the red line indicates the linear fit to the end points of each scan leg. This rough baseline subtraction also removed pixel-to-pixel offsets.

\subsection{First Step of Deglitching}

After this first-order baseline subtraction was applied, the multiresolution median transform (MMT) deglitching algorithm was implemented to remove the effects of cosmic ray hits. The details of the MMT algorithm implemented in HIPE have been described in the PACS data reduction guide and references therein. We used the recommended parameters for the PACS $160 \mu \mathrm{m}$ band, with nscales $=2$, nsigma $=5$, incr-fact $=2$, mode $=$ multiply. For the PACS $100 \mu \mathrm{m}$ band, we observed that applying those parameters led to the core of the PSF of bright point sources also being removed. Hence, we selected a more conservative set of parameters for the green band, with $n$ scales $=1$. This approach preserved more of the source flux at the expense of more glitches remaining in the PACS $100 \mu \mathrm{m}$ images. The residual glitches in the $100 \mu \mathrm{m}$ band therefore need to be removed at a later stage using the second level deglitching algorithm.

\subsection{Refined Baseline Subtraction}

The next step in the PACS data processing is to remove the contribution of $1 / f$ noise and baseline drifts more accurately than in the rough baseline subtraction. We developed our own method in IDL to remove $1 / f$ noise and drifts, which takes advantage of previous, lower resolution FIR observations of the Magellanic Clouds with IRAS at $100 \mu \mathrm{m}$ (Schwering 1989), Spitzer MIPS at $160 \mu \mathrm{m}$ (Meixner et al. 2006; Gordon et al. 2011) and DIRBE at $140 \mu \mathrm{m}$ and $240 \mu \mathrm{m}$ (Silverberg et al. 1993). The basic idea of the method is that the baseline of the PACS timelines can be estimated as the difference between the PACS timelines smoothed to the resolution of the MIPS or IRAS observations and a synthetic MIPS or IRAS timeline created from the coordinate timeline of the PACS frames and the MIPS or IRAS images. Here and below when we refer to the MIPS $160 \mu \mathrm{m}$ images, we mean a composite MIPS $160 \mu \mathrm{m}$ and DIRBE image. The areal coverage of the PACS $160 \mu \mathrm{m}$ image is larger than the MIPS $160 \mu \mathrm{m}$ image. We extended the MIPS $160 \mu \mathrm{m}$ image with an interpolated flux distribution derived from the DIRBE $140 \mu \mathrm{m}$ and $240 \mu \mathrm{m}$ images. Note that small differences in the central wavelengths between the PACS 100 and IRAS 100, or PACS 160 and MIPS 160, combined with color corrections are negligible (of order $2 \%$ of typical dust temperatures) and therefore cause only small discrepancies in the baseline subtraction.

In order to prepare synthetic PACS-like timelines, the MIPS $160 \mu \mathrm{m}$ and IRAS $100 \mu \mathrm{m}$ images of the LMC or SMC are first converted from MJy sr${ }^{-1}$ to Jy pixel $^{-1}$, where the size of the pixel corresponds to the size of the PACS $160 \mu \mathrm{m}$ bolometer (6."4), or of the PACS $100 \mu \mathrm{m}$ bolometer (3".2). A MIPS or IRAS synthetic timeline is then created as the value of the converted MIPS or IRAS image at the R.A./decl. location in each PACS $160 \mu \mathrm{m}$ frame of the timeline. The PACS $160 \mu \mathrm{m}$ and PACS $100 \mu \mathrm{m}$ timelines are then convolved using a $1 \mathrm{D}$ convolution to MIPS $160 \mu \mathrm{m}$ and IRAS $100 \mu \mathrm{m}$ resolutions, respectively. For the PACS $160 \mu \mathrm{m}$ band convolution, we use a slice through the center of the convolution kernel created by Aniano et al. (2011), while for the PACS $100 \mu \mathrm{m}$ band convolution, we use a slice through the center of a Gaussian convolution kernel of FWHM 


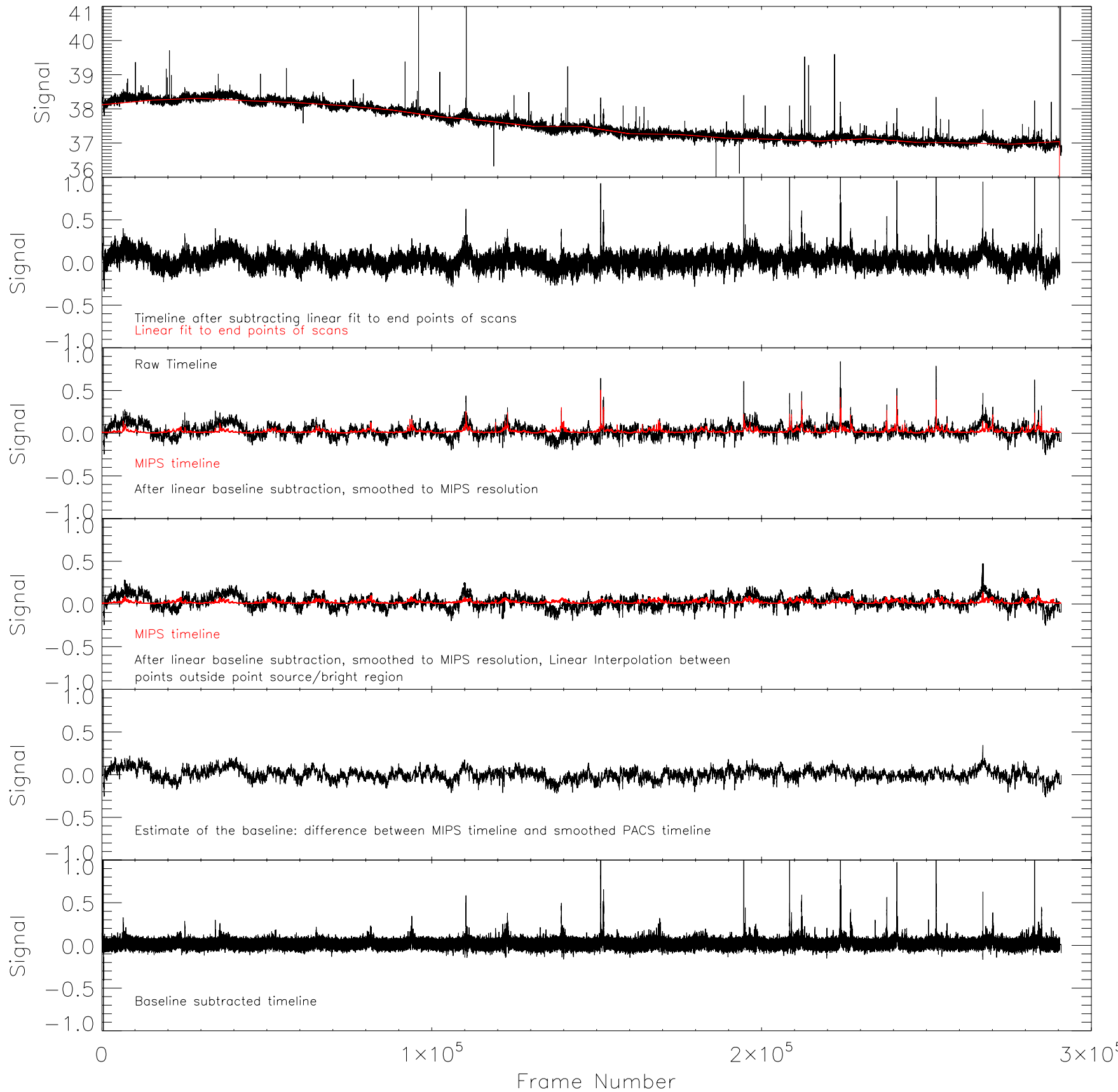

Figure 2. PACS timelines for one bolometer and during one AOR, at the different steps of the PACS processing algorithm. Starting from the top, the first panel shows the raw timeline in black, on which is over-plotted in red the linear fit to the end points of each scan leg, performed during the rough baseline estimate. The second panel shows the timeline resulting from this rough baseline subtraction. The third panel shows the timeline after initial rough baseline subtraction, smoothed to MIPS resolution using a 1D slice through the center of the 2D convolution kernel in black. The synthetic MIPS timeline obtained from the PACS mapping scheme and the MIPS map is shown in red. The fourth panel shows the MIPS timeline in red, and in black, the PACS timeline, after a rough baseline subtraction, smoothed to MIPS resolution, and after performing a linear interpolation inside masked bright and/or compact regions. The fifth panel shows the final estimation of the residual baseline (i.e., after rough baseline removal from a linear fit to the end points of each scan leg), obtained by subtracting the MIPS timeline to the smoothed, interpolated PACS timeline in the fourth panel. The final, baseline subtracted PACS timeline is shown in the bottom panel.

(A color version of this figure is available in the online journal.)

width $\left.\sqrt{(} \mathrm{FWHM}_{I R A S}^{2}-\mathrm{FWHM}_{\mathrm{PACS}}^{2}\right)$, where $\mathrm{FWHM}_{I R A S}=4.3$ is the FWHM resolution of the IRAS $100 \mu \mathrm{m}$ image, and $\mathrm{FWHM}_{\mathrm{PACS}}=8^{\prime \prime}$ is the resolution of the PACS $100 \mu \mathrm{m}$ image. An example of smoothed PACS timelines is shown in the third panel of Figure 2.

Smoothing the timelines in 1D was not equivalent to extracting a 1D timeline from a 2D smoothed map but was necessary to boot-strap our way to the first 2D PACS maps. Nevertheless, our approach caused some artifacts in regions with bright and/or compact sources that required some special attention. Because the convolution occurred in 1D, the signal "dilution" is not as high as in the 2D convolution case. As a result, the shape of bright and/or compact sources in the smoothed PACS timelines did not match the shape of the same sources in the MIPS $160 \mu \mathrm{m}$ or IRAS $100 \mu \mathrm{m}$ timelines. This effect was manifest in Figure 3, which showed a zoom on a point source in PACS $160 \mu \mathrm{m}$ and MIPS $160 \mu \mathrm{m}$ timelines. Because the baseline was estimated as the difference between the smoothed PACS timelines and 


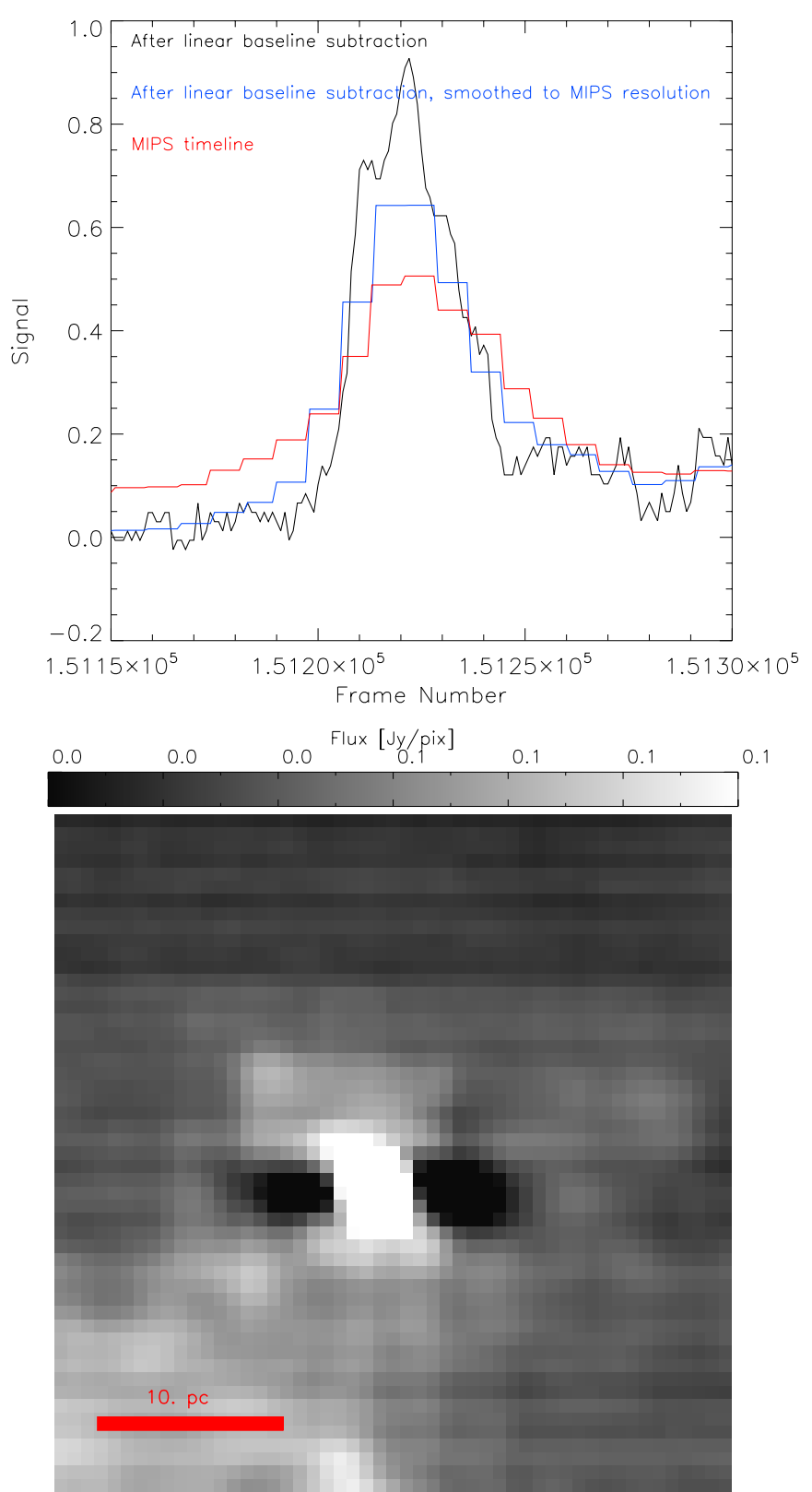

Figure 3. Baseline subtraction and point sources. Top: example of point source morphology mismatch between the smoothed PACS and the MIPS $160 \mu \mathrm{m}$ timelines. The black line shows the roughly baseline subtracted PACS timeline, corresponding to the timeline in the second panel in Figure 2. The red line shows the MIPS timeline, as in the third panel of Figure 2. The blue line shows the roughly baseline subtracted, smoothed PACS timeline, as panel 3 of Figure 2. Bottom: effects in the projected maps of over-subtraction around bright/compact sources due to differences in the shapes of the MIPS and PACS timelines caused by the one-dimensionality of the convolution. This problem is solved by linearly interpolating the timeline inside such regions.

(A color version of this figure is available in the online journal.)

the MIPS $160 \mu \mathrm{m}$ or IRAS $100 \mu \mathrm{m}$ timelines, this mismatch therefore resulted in over-subtraction lobes on either side of a compact or bright source, as shown in Figure 3. Maps of the subtracted baseline showed that this problem also affected the flux of more extended sources in a subtler way. To avoid this issue, we masked point, compact, and bright extended regions in the baseline subtraction process. The masks are shown in Figure 6, as an example for the LMC. To estimate the baseline inside those regions, the smoothed PACS and the MIPS $160 \mu \mathrm{m}$ or IRAS $100 \mu \mathrm{m}$ timelines were linearly interpolated between the signal in the frames located right outside a given region, and the baseline was estimated as the difference between the interpolated, smoothed PACS and MIPS $160 \mu \mathrm{m}$ or IRAS $100 \mu \mathrm{m}$ timelines. Outside the masked regions, the baseline was simply estimated as the difference between the smoothed PACS timelines and the MIPS $160 \mu \mathrm{m}$ or IRAS $100 \mu \mathrm{m}$ timelines. This process was illustrated in the fourth panel of Figure 2. The baseline was subtracted from the PACS timelines. An example of baseline subtracted PACS timeline is shown in the bottom panel of Figure 2.

\subsection{Removal of Long Glitches in the PACS $100 \mu \mathrm{m}$ Band}

We found the presence of discontinuities in the brightness images for the $100 \mu \mathrm{m}$ band (see Figure 5). These discontinuities were caused by jumps in the timelines affected by cosmic rays and affect an entire line of the PACS $100 \mu \mathrm{m}$ detector. These so-called long glitches were not removed by the deglitching algorithms implemented in HIPE (MMT and second level deglitching). We therefore developed a custom IDL algorithm to remove these artifacts. First, the rough position of the glitches was estimated by recording their coordinates in the PACS images. Second, the total signal in the timeline of the detector was computed, and each timeline around the coordinates of the jump was scanned by eye. The shape of the jump in the total timeline was obvious, as shown in Figure 6, allowing a determination of the affected line's location (a.k.a. index). To determine the index of the frame at which the glitch occurred, we computed the difference between the timelines and the timelines shifted by 1 and 2 frames. The index of the jump was computed as the index where this difference is greater than $0.2 \mathrm{Jy} \mathrm{pixel}^{-1}$. The sections of the timelines on the left and right side of the long glitch, which show a characteristic ramp caused by the cosmic ray hit, were fit to a polynomial. The fitting was done iteratively in order to try different orders of polynomial and lengths around the glitch to perform the fitting. Once a reasonable order and length for the fit were identified, typically a polynomial of order two and a fit 150 frames long on either side of the glitch, the fit was removed from the timeline of each bolometer in the affected detector line around the glitch. The result of this algorithm was shown in the right panel of Figure 5.

\subsection{Correction of Astrometric Offsets}

At the time of the observations, the Herschel spacecraft still suffered from degraded pointing reconstruction performance. This degraded pointing caused astrometric offsets of up to $5^{\prime \prime}-10^{\prime \prime}$ in the PACS images, which is significantly larger than the nominal astrometric accuracy of Herschel is $2^{\prime \prime}$ at the $2 \sigma$ level. ${ }^{37}$ These astrometric offsets posed problems not only in the combination of the different epochs available (e.g., smearing of the PSF, double sources, etc.), but also in the combination of the HERITAGE images with previous Spitzer images of the Magellanic Clouds (Meixner et al. 2006; Gordon et al. 2011). Therefore, we developed an algorithm (in IDL) to correct those astrometric offsets based on the positions of point sources in the MIPS $24 \mu \mathrm{m}$ catalog, the astrometric accuracy of which is tied to 2MASS and accurate to $\sim 0$ '. 3 (Skrutskie et al. 2006). We chose to use the $160 \mu \mathrm{m}$ band of PACS rather than its $100 \mu \mathrm{m}$ counterpart because the $160 \mu \mathrm{m}$ band is deeper and because the baseline removal is superior for that band, being based on the MIPS 160 map at higher resolution than the IRAS 100 map.

\footnotetext{
37 http://herschel.esac.esa.int/Docs/PACS/html/ch03s06.html
} 

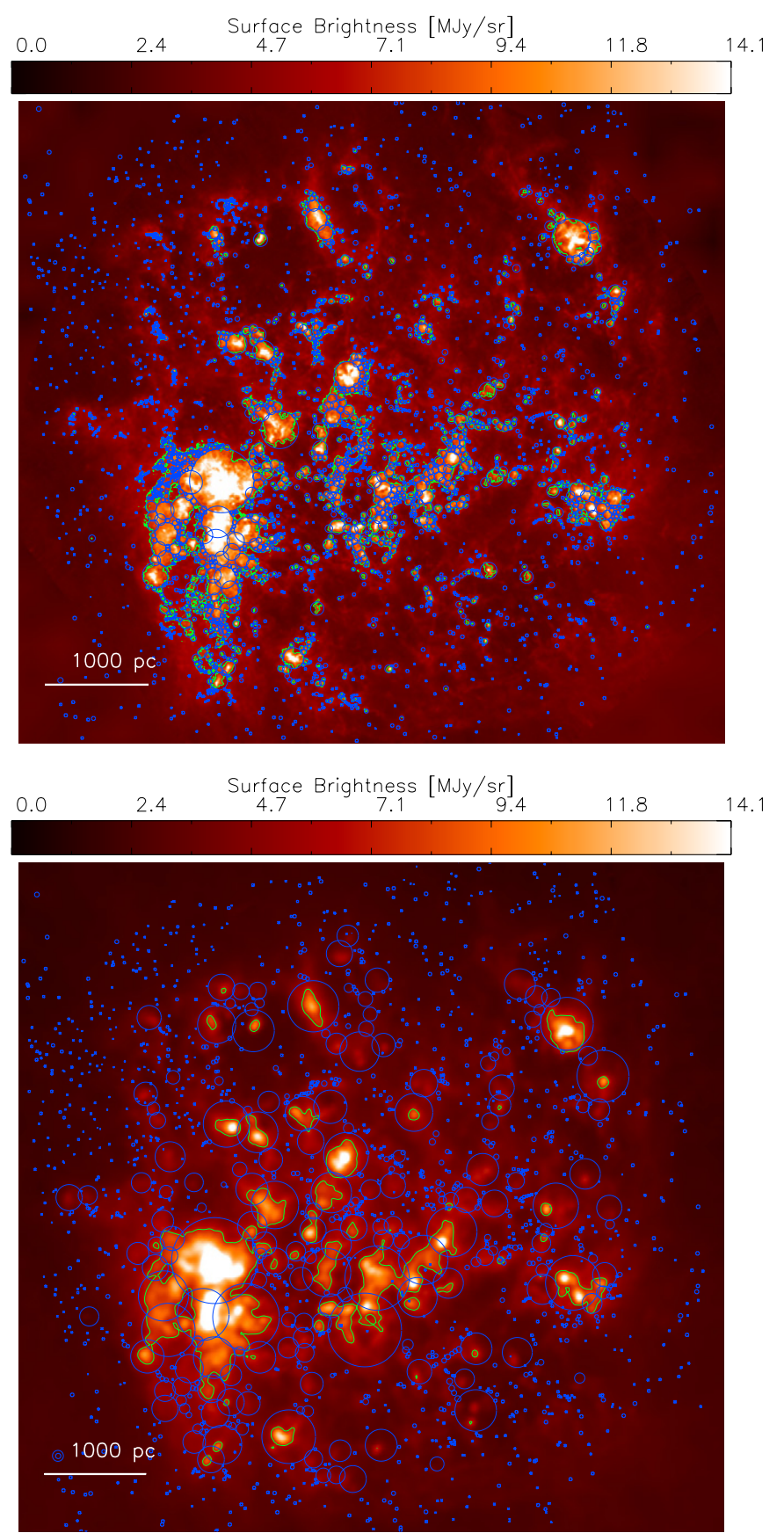

Figure 4. Spitzer MIPS $160 \mu \mathrm{m}$ (top) and IRAS $100 \mu \mathrm{m}$ (bottom) maps of the LMC. The masks used to interpolate the PACS timelines inside bright or compact sources in the LMC during the baseline estimate are overlaid as blue circles. The $50 \mathrm{MJy} \mathrm{sr}^{-1}$ surface brightness contour, corresponding to the transition to the nonlinear regime of MIPS $160 \mu \mathrm{m}$ is shown in green in the MIPS image.

First, each scan leg of each AOR in the PACS $160 \mu \mathrm{m}$ data was mapped to spatial coordinates in R.A. and decl. using the PhotProject algorithm in HIPE. PSF fitting was performed on each scan leg image using the model PSF, and a PACS $160 \mu \mathrm{m}$ point source list was subsequently extracted. For each scan leg, the PACS $160 \mu \mathrm{m}$ point source list was cross-correlated with the list of point sources identified in the MIPS $24 \mu \mathrm{m}$ catalog. The (constant) astrometric offset for each scan leg was then calculated as the median difference between the point source coordinates in the PACS $160 \mu \mathrm{m}$ images and in the MIPS $24 \mu \mathrm{m}$ images. The astrometric offset was finally subtracted from the coordinates of the frames. As a final check on the astrometric
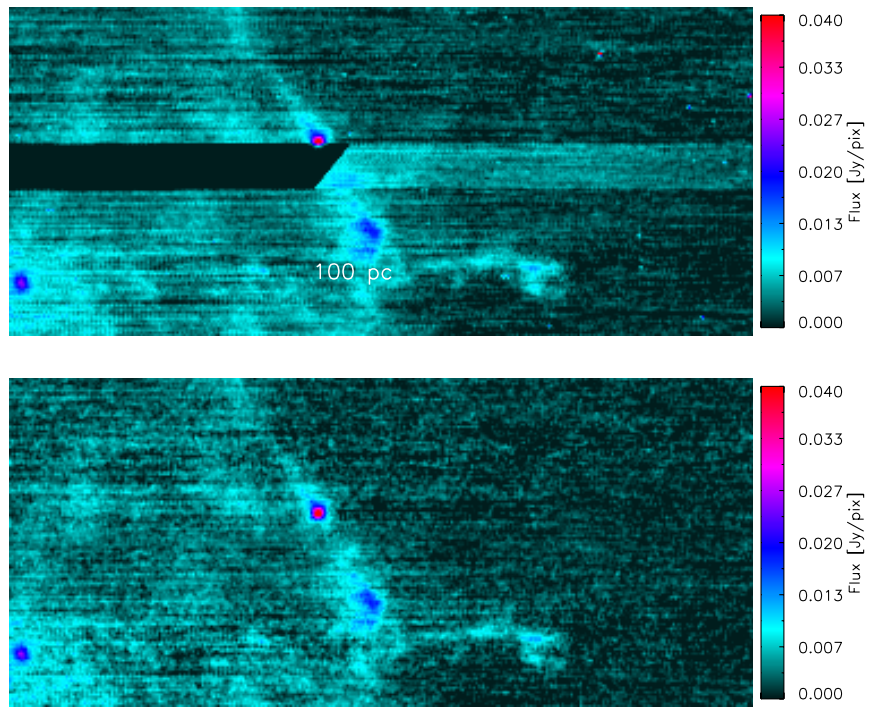

Figure 5. Example of a long glitch, a cosmic ray hit affecting an entire line of bolometers in the PACS $100 \mu \mathrm{m}$ detector. The top panel shows the image of the glitch, while the bottom panel shows the projected timelines after the long glitch has been removed by our custom algorithm.

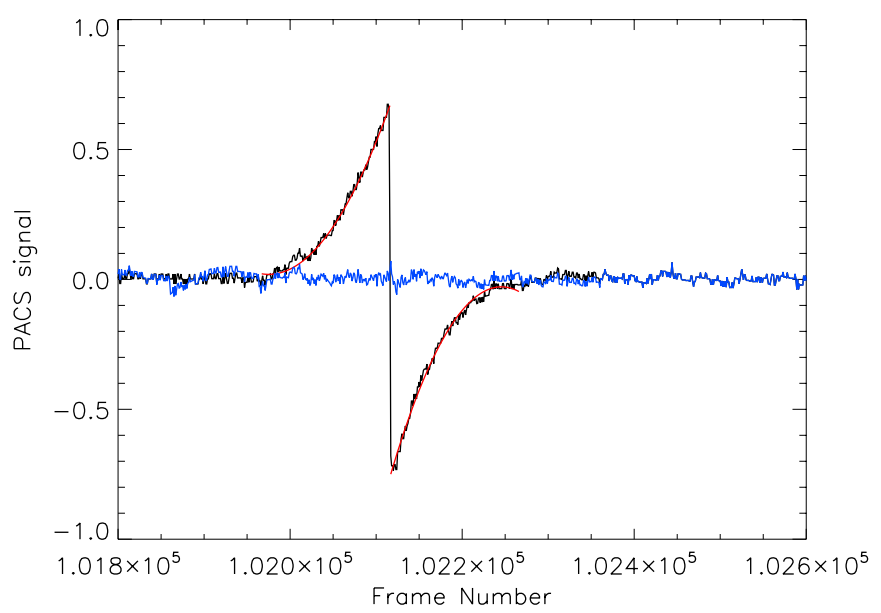

Figure 6. Manifestation of a long glitch in the PACS $100 \mu \mathrm{m}$ timelines. The black line shows the uncorrected timeline, with the discontinuity at frame number $\simeq 1.021 \times 10^{5}$ caused by the glitch. The red line corresponds to a polynomial fit of order two to the 150 frames on each side of the discontinuity. The blue line shows the corrected timeline (glitch removed).

correction, we extracted sources from the PACS 100 and $160 \mu \mathrm{m}$ images (Section 7 below) and did another comparison to the MIPS $24 \mu \mathrm{m}$ source locations. We found a small $\left(0.5-1{ }^{\prime \prime} .7\right)$ but systematic offset between the PACS sources and the MIPS $24 \mu \mathrm{m}$ sources in the R.A. and decl. directions that we corrected for both the PACS 100 and $160 \mu \mathrm{m}$ images. Figure 7 shows the histogram of the source offsets between PACS 160 and MIPS 24 found in the LMC and SMC after the correction. The final astrometry of the PACS images is consistent both with the MIPS $24 \mu \mathrm{m}$ and by extension the SAGE surveys, and with the SPIRE data sets.

\subsection{Mapping and Second Level Deglitching}

As pointed out in Section 3.4, residual glitches subsisted in the PACS $100 \mu \mathrm{m}$ images. Those were eliminated by applying the second level deglitching algorithm in HIPE. We used the sigma-clipping algorithm in median mode with a box size of 10 and a sigma threshold of 3 . The PACS $160 \mu \mathrm{m}$ images were 

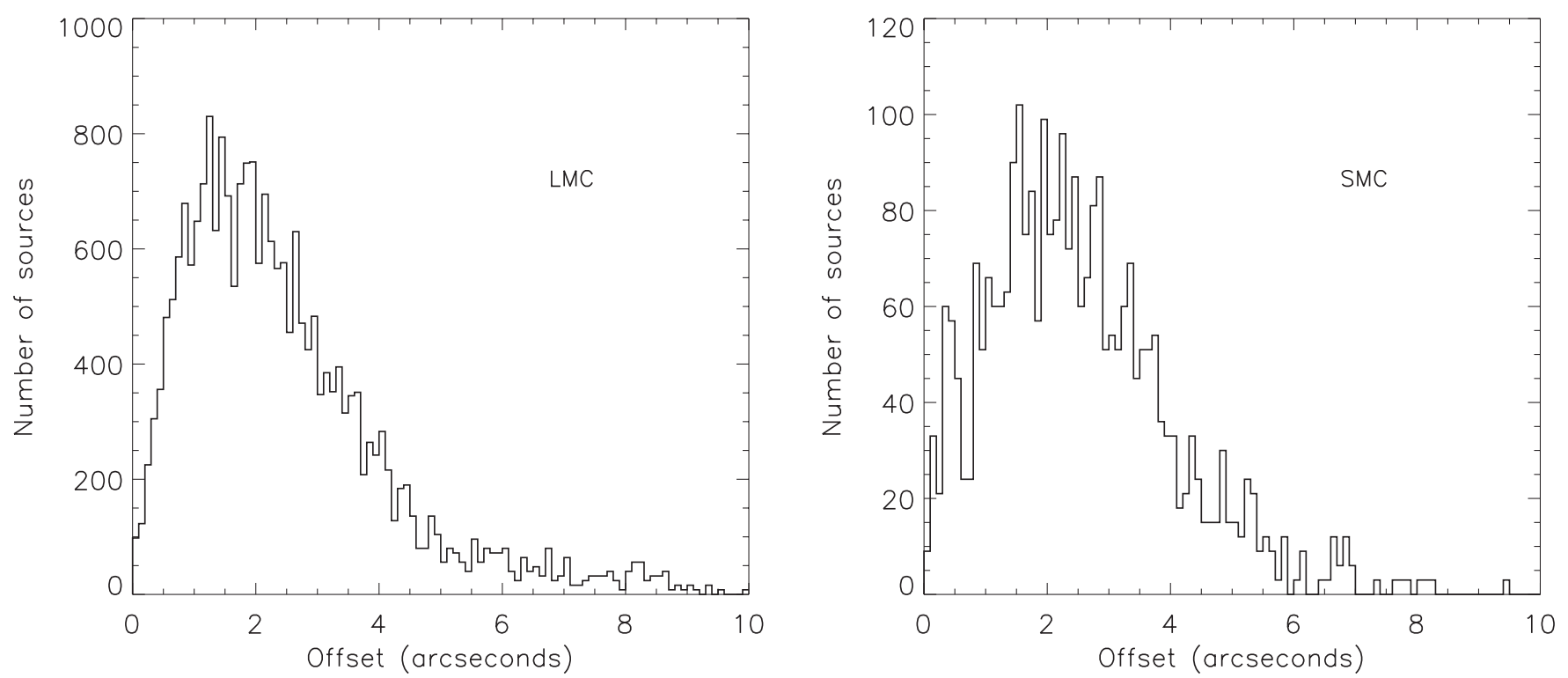

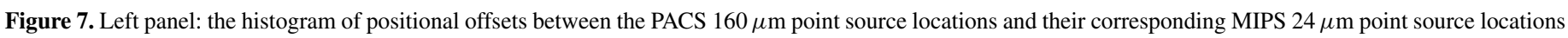
for the LMC. Right panel: similar positional histogram offsets but for the SMC.

Table 3

Principal Characteristics of the HERITAGE Survey, Herschel Program ID KPOT_mmeixner_1: Wavelengths, Resolution, and Sensitivity

\begin{tabular}{|c|c|c|c|c|c|}
\hline \multirow[t]{2}{*}{ Characteristic } & \multicolumn{2}{|c|}{$\operatorname{PACS} \lambda(\mu \mathrm{m})$} & \multicolumn{3}{|c|}{$\operatorname{SPIRE} \lambda(\mu \mathrm{m})$} \\
\hline & 100 & 160 & 250 & 350 & 500 \\
\hline Instrumental pixel size $\left({ }^{\prime \prime}\right)$ & 3.2 & 6.4 & 6 & 10 & 14 \\
\hline Image pixel size $(")$ & 2 & 3 & 6 & 10 & 14 \\
\hline Angular resolution, FWHM $(")$, P.A. $\left({ }^{\circ}\right)$ & $6.7 \times 6.9,62.3$ & $10.7 \times 12.1,9.3$ & 18.2 & 24.9 & 36.3 \\
\hline Sensitivity, $10 \sigma\left(\mathrm{MJy} \mathrm{sr}^{-1}\right)$ & $85-90^{\mathrm{a}}$ & $32-50^{\mathrm{a}}$ & 6 & 3 & 2 \\
\hline
\end{tabular}

Note. ${ }^{a}$ The noise in PACS varies in the map with masked regions having the higher noise values than the unmasked regions, see text (Section 3.10) for details.

successfully deglitched using the MMT algorithm, so this step was unnecessary for the $160 \mu \mathrm{m}$ band. The deglitched, baseline subtracted, and astrometrically corrected PACS $160 \mu \mathrm{m}$ and $100 \mu \mathrm{m}$ timelines were mapped using the PhotProject task in HIPE. Maps for the LMC were created from the combination of both epoch 1 and 2 data. For the SMC, maps were created by combining the data from epochs $1-3$. The limiting surface brightnesses listed in Table 3 represent $10 \sigma$ of the standard deviations measured on the outer regions of the images where there is no significant signal. For both the LMC and SMC, we used the individual epoch maps to understand the uncertainties for the source extractions from the PACS images.

\subsection{Cross-calibration of PACS 100 and $160 \mu \mathrm{m}$ with Prior Missions}

We examined the accuracy of the absolute flux calibration with PACS. For this purpose, we convolved and resampled the PACS $160 \mu \mathrm{m}$ (or PACS $100 \mu \mathrm{m}$ ) images to the same resolution and grid as the MIPS $160 \mu \mathrm{m}$ (or IRAS $100 \mu \mathrm{m}$ ) images. For the convolution to a common resolution, we used the convolution kernels provided by Aniano et al. (2011) for the PACS $160 \mu \mathrm{m}$ images, and a Gaussian kernel of FWHM 4.3 (IRAS $100 \mu \mathrm{m}$ resolution) for the PACS $100 \mu \mathrm{m}$ images. We evaluated and applied color corrections for PACS, MIPS, and IRAS, and found respectively $0.97,0.99,0.97$, and 0.96 for PACS 100, PACS 160, MIPS 160, and IRAS 100 respectively, assuming a modified blackbody with temperature $25 \mathrm{~K}$ and emissivity spectral index $\beta=2$. We then performed a pixel-to-pixel correlation between the PACS and MIPS/IRAS flux to check for gain differences.

We found that PACS and MIPS $160 \mu \mathrm{m}$ agree within $4 \%$ for surface brightnesses below $50 \mathrm{MJy} \mathrm{sr}^{-1}$. The top panel of Figure 8 shows the pixel-to-pixel correlation between the PACS $160 \mu \mathrm{m}$ and MIPS $160 \mu \mathrm{m}$ maps. A bisector linear fit to the correlation using only the data below $50 \mathrm{MJy} \mathrm{sr}^{-1}$ yielded a slope of 0.96 (i.e., PACS $=0.96$ MIPS), shown with a green line in Figure 8. Note, that, without applying any color correction, this slope becomes 1.00. The agreement is expected since (1) the baseline subtraction algorithm effectively sets the calibration of the PACS maps to the MIPS calibration outside of the regions used to mask bright and/or compact FIR emission in the PACS timelines (see Section 3.5), and (2) those regions roughly correspond to the $50 \mathrm{MJy} \mathrm{sr}^{-1}$ contour of the MIPS 160 map (Figure 4). Note that color corrections were not applied during the baseline subtraction process, which explains why the slope of the MIPS 160/PACS 160 pixel-to-pixel correlation is closer to 1 when no color corrections are applied. Although the match between the PACS 160 and MIPS 160 flux calibration below $50 \mathrm{MJy} \mathrm{sr}^{-1}$ is built-in our baseline subtraction technique, Paladini et al. however showed in a report on the PACS extended flux calibration (https://nhscsci.ipac.caltech.edu/pacs/docs/Photometer/ PICC-NHSC-TR-034.pdf) based on PACS maps processed with Scanamorphos or MADmap, that PACS 160 and MIPS 160 were in agreement within 5\%-20\% below $50 \mathrm{MJy} \mathrm{sr}^{-1}$. 

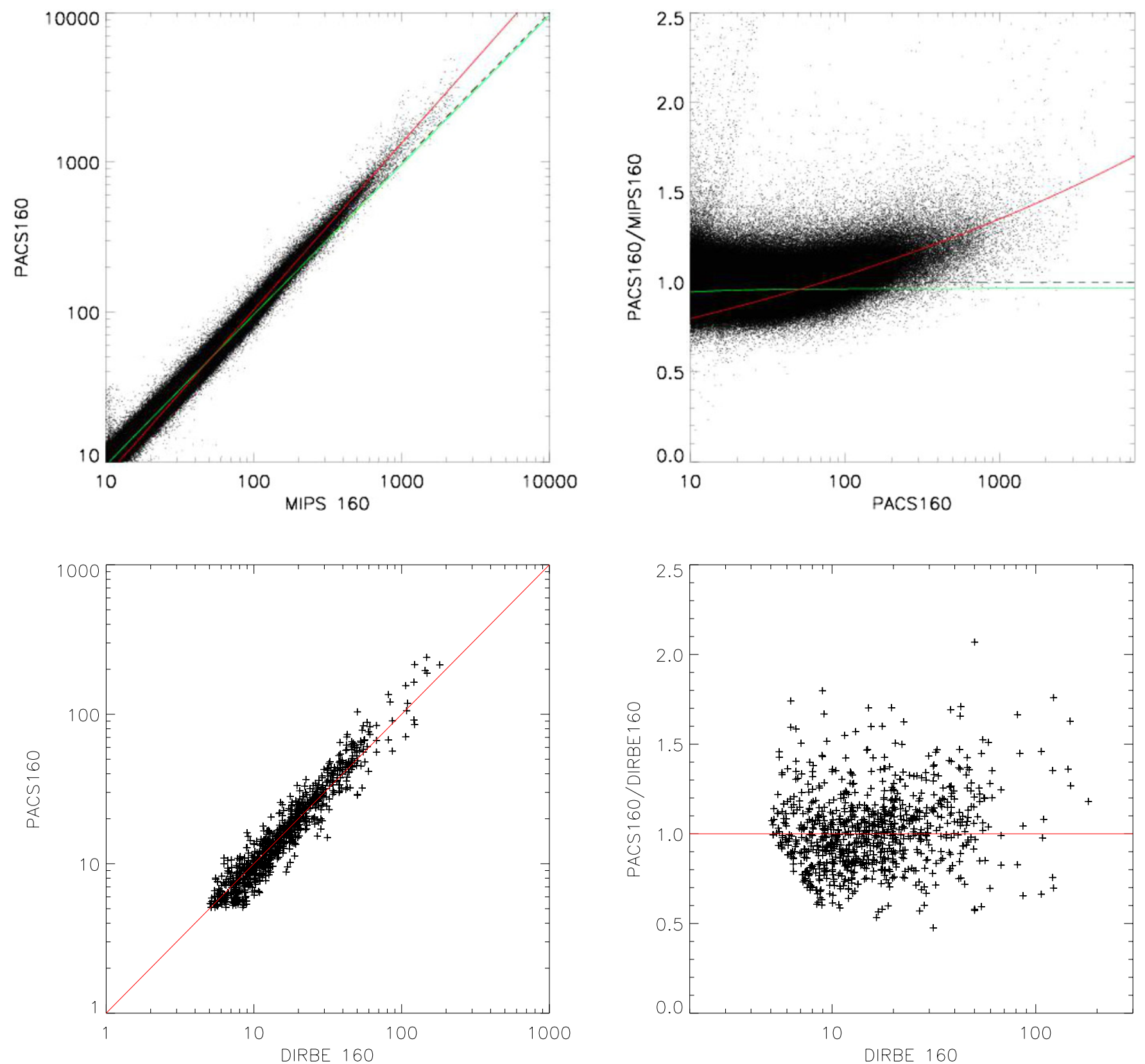

Figure 8. Pixel-to-pixel surface brightness correlation between the combined epochs of the PACS $160 \mu \mathrm{m}$ convolved, resampled images of the LMC, and the MIPS $160 \mu \mathrm{m}$ on the one hand (top), and the interpolation at $160 \mu \mathrm{m}$ between the DIRBE 140 and $240 \mu \mathrm{m}$ bands on the other hand (bottom). Surface brightnesses are plotted in $\mathrm{MJy} \mathrm{s}^{-1}$. In the top panel, the green line shows a bisector linear fit, of slope 0.96 , to the correlation in the linear range of the MIPS detector (surface brightness below $50 \mathrm{MJy} \mathrm{sr}^{-1}$ ). The red line shows an exponential fit to the correlation in the nonlinear range of MIPS (surface brightness above $50 \mathrm{MJy} \mathrm{sr}^{-1}$ ), which yields $\left(\mathrm{PACS} / 50 \mathrm{MJy} \mathrm{sr}^{-1}\right)=0.96\left(\mathrm{MIPS} / 50 \mathrm{MJy} \mathrm{sr}^{-1}\right)^{1.11}$ in the nonlinear regime. In the bottom panel, the red line shows a 1:1 correlation.

(A color version of this figure is available in the online journal.)

Above a brightness threshold of $50 \mathrm{MJy} \mathrm{sr}^{-1}$, which corresponds to regions inside the masks described in Section 3.5, there was a departure from a linear correlation between MIPS and PACS $160 \mu \mathrm{m}$ : the MIPS $160 \mu \mathrm{m}$ brightness was lower than the PACS $160 \mu \mathrm{m}$ brightness. Recall that, because the baseline was linearly interpolated within the masks marking the $50 \mathrm{MJy} \mathrm{sr}^{-1}$ contour of MIPS 160 and was not based on the MIPS synthetic timelines, a match between the MIPS 160 and PACS 160 is not necessarily expected in this case. The flux calibration inside those regions is left to the PACS calibration. The PACS response function is known to be linear up to $10^{4} \mathrm{MJy} \mathrm{sr}^{-1}$, while MIPS is linear only up to $50 \mathrm{MJy} \mathrm{sr}^{-1}$. Thus this departure between the PACS and MIPS flux calibrations above $50 \mathrm{MJy} \mathrm{sr}^{-1}$ was most likely due to the nonlinearity of the MIPS detector. A power-law fit between the PACS and MIPS $160 \mu \mathrm{m}$ brightnesses for the flux ranges above $50 \mathrm{MJy} \mathrm{sr}^{-1}$ gave $\left(\mathrm{PACS} / 50 \mathrm{MJy} \mathrm{sr}^{-1}\right)=0.96\left(\mathrm{MIPS} / 50 \mathrm{MJy} \mathrm{sr}^{-1}\right)^{1.11}(\mathrm{red}$ line in Figure 8). Because the flux calibration of PACS inside the $50 \mathrm{MJy} \mathrm{sr}{ }^{-1}$ contour of MIPS 160 is not tied to the flux calibration of MIPS 160, the MIPS nonlinearity was therefore not propagated to the PACS maps via the baseline removal algorithm.

Previous COBE DIRBE observations of the LMC and SMC provide an additional, independent way to validate the flux calibration of our PACS $\mu \mathrm{m}$ maps. Although DIRBE does not have a $160 \mu \mathrm{m}$ band, we estimated the brightness at $160 \mu \mathrm{m}$ 

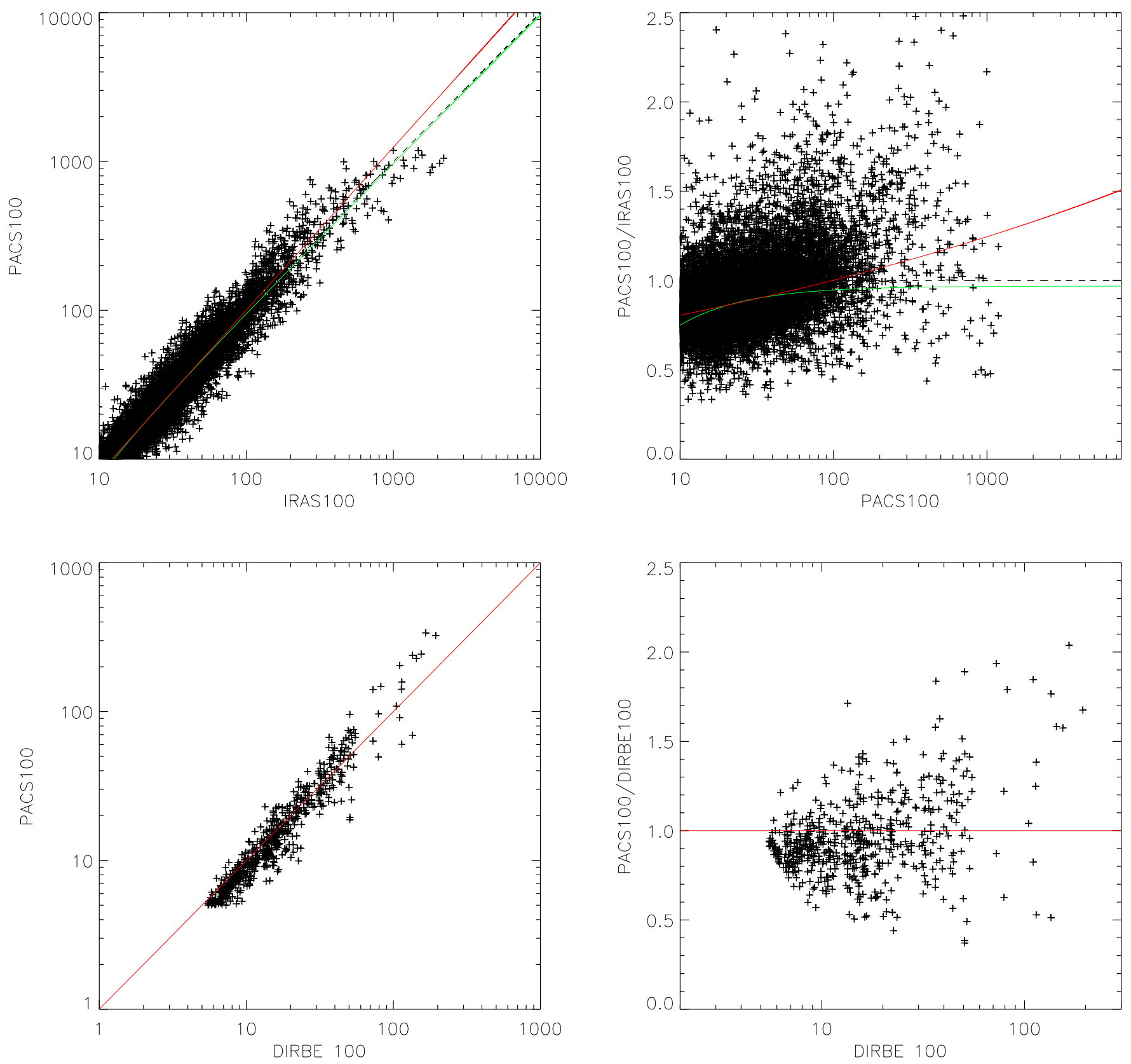

Figure 9. Pixel-to-pixel correlation between the convolved, resampled PACS $100 \mu \mathrm{m}$ combined map of the LMC and the IRAS $100 \mu \mathrm{m}$ image on the one hand (top) and the DIRBE $100 \mu \mathrm{m}$ map on the other hand (bottom). Surface brightnesses are plotted in $\mathrm{MJy} \mathrm{sr}^{-1}$. In the top panel, the green line shows a bisector linear fit to the correlation, yielding a slope of 0.97 . The red line shows an exponential fit, leading to $\left(\mathrm{PACS} / 50 \mathrm{MJy} \mathrm{sr}^{-1}\right)=0.94\left(I R A S / 50 \mathrm{MJy} \mathrm{sr}^{-1}\right)^{1.09}$. In the bottom panel, the red line indicates a 1:1 correlation.

(A color version of this figure is available in the online journal.)

by performing a linear interpolation in the log-brightness of the 140 and $240 \mu \mathrm{m}$ bands. After applying the proper color corrections, we analyzed the pixel-to-pixel correlation between the DIRBE interpolation at $160 \mu \mathrm{m}$ and the PACS $160 \mu \mathrm{m}$ brightness, which is shown in the bottom panel of Figure 8 . The red line shows a 1:1 correlation. Despite the large scatter, presumably caused by the peculiar sampling scheme of the DIRBE maps, the flux calibration of PACS $160 \mu \mathrm{m}$ appeared to be consistent with the flux calibration of DIRBE across the whole brightness range. This is an independent confirmation that our PACS maps are well calibrated, since the baseline removal was in no way tied to DIRBE. In addition, $C O B E$ was calibrated on an extended source (the cosmic microwave background $(\mathrm{CMB}))$ and therefore provides the best extended calibration source.

We performed a similar analysis on the PACS $100 \mu \mathrm{m}$ band. The pixel-to-pixel correlation between the IRAS $100 \mu \mathrm{m}$ and PACS $100 \mu \mathrm{m}$ maps is shown in the top panel of Figure 9. There was good agreement between the PACS and IRAS calibration. A bisector linear fit to the correlation over the whole flux range yielded PACS $=0.97$ IRAS-2.18 $\mathrm{MJy} \mathrm{sr}^{-1}$. An exponential fit over the whole flux range yielded $\left(\mathrm{PACS} / 50 \mathrm{MJy} \mathrm{sr}{ }^{-1}\right)=0.94\left(\text { IRAS/50 } \mathrm{MJy} \mathrm{sr}^{-1}\right)^{1.09}$. So there was a slight nonlinearity in this pixel-to-pixel correlation. We also examined the pixel-to-pixel correlation between the DIRBE $100 \mu \mathrm{m}$ maps, and the correspondingly re-convolved, 

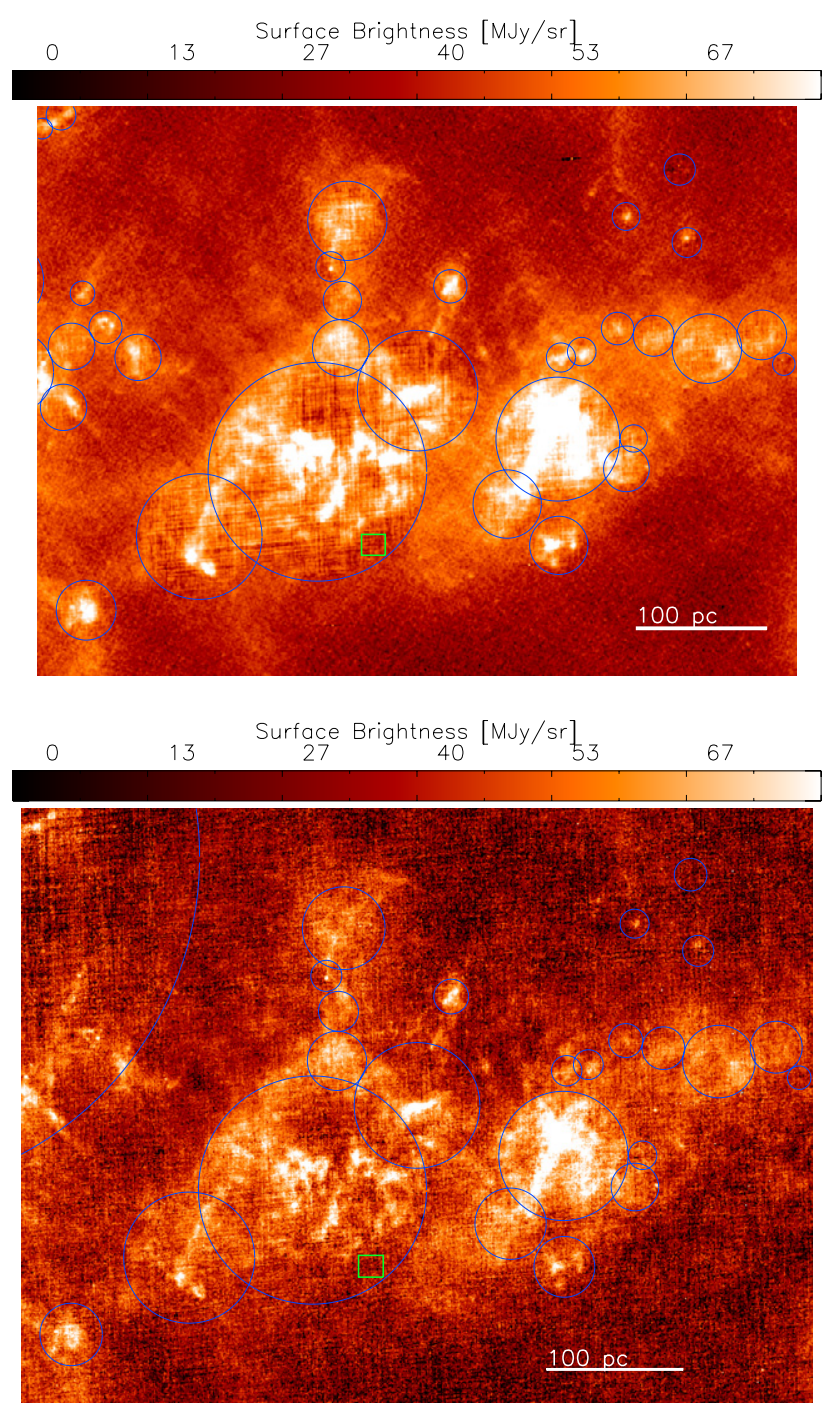

Figure 10. Zoom-in on the PACS 160 (top) and 100 (bottom) $\mu \mathrm{m}$ maps of the LMC. The blue circles correspond to masks used to estimate the $1 / f$ noise and bolometer drifts (Section 3.5, Figure 4). The green box shows an example of region used to estimate the residual noise in masked regions of the LMC.

resampled PACS maps (see bottom panel of Figure 9). The pixel-to-pixel correlation between the final PACS $100 \mu \mathrm{m}$ maps and the DIRBE $100 \mu \mathrm{m}$ was consistent with a 1:1 correlation.

\subsection{Noise Estimation}

Because the $1 / f$ noise is estimated and removed in different ways inside and outside the masks define by the $50 \mathrm{MJy} \mathrm{sr}^{-1}$ contour of MIPS 160, residual noise in the PACS maps is not homogeneous. We have estimated the residual noise in those different cases by computing the standard deviation of the PACS brightness in several regions, inside masks but outside of obviously visible ISM structure on the one hand (see example shown as a green box in Figure 10), and outside masks in the outskirts of the Magellanic Clouds on the other hand. For both the $\mathrm{LMC}$ and SMC, we found a $1 \sigma \mathrm{rms}$ of $\simeq 5 \mathrm{MJy} \mathrm{sr}^{-1}$ and $9 \mathrm{MJy} \mathrm{sr}^{-1}$ in masked regions, and $3.2 \mathrm{MJy} \mathrm{sr}^{-1}$ and 8.5 $\mathrm{MJy} \mathrm{sr}^{-1}$ outside of the masks for the 160 and $100 \mu \mathrm{m}$ respectively. Residual $1 / f$ noise appears as striping in the PACS maps. The difference in the quality of the $1 / f$ noise removal between masked and unmasked regions is obvious in Figure 10: the striping is of larger amplitude in the masked compared to the unmasked regions. Outside the masks, the $1 / f$ noise is estimated as the difference between PACS 160 (resp. PACS 100) timelines smoothed to MIPS 160 (resp. IRAS 100) resolution. Thus, the $1 / f$ noise is removed down to size scales comparable to the MIPS 160 (respectively IRAS 100) resolution for the 160 and $100 \mu \mathrm{m}$ bands. Inside the masks, the $1 / f$ noise is calculated as the difference between linear interpolations of the smoothed PACS and MIPS timelines, where the points used to perform the linear interpolation are located right outside the mask. Inside the masked regions, the $1 / f$ noise is therefore corrected on length scales greater or equal to the radius of the mask, which is larger than the resolutions of MIPS 160 and IRAS 100 um bands. Since the amplitude of $1 / f$ noise increases with size scales, the residual $1 / f$ noise, and hence striping, inside the masked regions is significantly larger than outside the masked regions.

\subsection{Limitations and Benefits}

Due to the peculiar nature of the removal of drifts and $1 / f$ noise in the HERITAGE data, there are limitations to the possible applications of this product. In particular, the noise properties are inhomogeneous. This precludes studies of the power-spectrum (or similar) of the ISM. In addition, residual striping makes the study of the structure of compact objects, such as $\mathrm{H}$ II regions and supernovae remnants, difficult. Nonetheless, we have derived a technique based on filtering of the Fourier spectrum to remove this residual striping on small compact regions. In the 2D Fourier spectrum of a small portion (a few arcmin in side) of the PACS maps, the striping very clearly appears as a line perpendicular to the direction of the striping in the image. After being identified in the Fourier spectrum, the striping can be inverse Fourier transformed, and subtracted from the image. This technique has been successfully implemented in Matsuura et al. (2011) and Otsuka et al. (2010), and does not affect the flux calibration. Additionally, we are currently developing an algorithm, Tamasis (P. Chanial et al., in preparation), similar to Scanamorphos or MADmap, to remove $1 / f$ noise in the PACS timelines. Processing the HERITAGE data through Tamasis requires significant time on a super-computer, which makes the testing and development of this software a lengthy process. At the moment, the accuracy of the flux calibration and different artifacts present in the resulting maps are being worked on. We will provide Tamasis maps to the community in the future.

As a check on our approach, we have applied both our $1 / f$ noise removal algorithm and Scanamorphos to publicly available maps of M31 (taken as part of the Herschel Exploitation of Local Galaxy Andromeda; Fritz et al. 2012), and other galaxies from the KINGFISH (Key Insights on Nearby Galaxies: a Far-Infrared Survey with Herschel; Kennicutt et al. 2011) and SINGS (Spitzer Infrared Nearby Galaxies Survey; Kennicutt et al. 2003) samples, to check whether our custom algorithm introduced flux calibration issues via the tie to previous observations with different detector technologies. We found the PACS flux calibration resulting from both algorithms were correlated with a 1:1 relation, and that the PACS flux calibration of maps reduced with Scanamorphos, independently from Spitzer, $I R A S$, or DIRBE, was in excellent agreement (10\%) with those previous missions.

Because the baseline estimation and removal are based on previous FIR observations of the Magellanic Clouds with Spitzer and IRAS, potential calibration issues in Spitzer and IRAS may be propagated in the PACS maps. However, we have verified that the flux calibration of the HERITAGE PACS maps is consistent with previous measurements (COBE DIRBE, Spitzer, IRAS) 
in regions where the baseline is estimated independently from those previous observations (Section 3.5). Independent studies (e.g., Paladini et al. 2012) have also shown that the PACS flux calibration is consistent within 5\%-20\% with in the more "diffuse" brightness regime (i.e., not masked in our baseline estimation). We emphasize that the Herschel PACS observations constitute tremendous progress compared to the previous Spitzer ones, thanks to the improved spatial resolution (a factor of 3.5 improvement for the $160 \mu \mathrm{m}$ band). Our baseline subtraction algorithm does not affect the native PACS resolution in any way.

\section{SPIRE PROCESSING}

The SPIRE images of the Magellanic Clouds were reduced using version 7 of HIPE, with the addition of customized routines to subtract the background, adjust the astrometry, identify glitches, remove residuals from temperature drifts, and mask discrepant data.

\subsection{Processing from Level 0.5 to Level 1}

We developed an algorithm to identify "concurrent glitches," i.e., glitches that affect all bolometers of a given SPIRE array simultaneously, which were most probably due to impact of cosmic rays on the bolometers' silicon support. We donated this algorithm to the SPIRE Instrument Control Center (ICC), and it was included in the standard pipeline.

We observed that, during strong temperature variations in the SPIRE cryo-cooler, the relation between the drift of the bolometers' signals and the plate temperature had a slightly different slope compared to the standard relation. For this reason, the temperature drift correction implemented in the standard pipeline left residual drifts in the signal (Figure 11). These residuals were significant in our data, especially in the $500 \mu \mathrm{m}$ band, given the length of the scans $\left(\sim 9^{\circ}\right.$ in the LMC). We derived new temperature drift coefficients for scans performed during strong cooler temperature variations measuring the thermistor voltages and the bolometer signals at each end of the scans. This approach reduced the residual drifts considerably.

\subsection{Astrometry Correction}

We corrected the astrometry of SPIRE data using an approach similar to the one we utilized for the PACS data (see Section 3.7) except on an AOR basis as opposed to a scan leg basis. We used the MIPS $24 \mu \mathrm{m}$ source catalogs of the LMC (Meixner et al. 2006) and the SMC (Gordon et al. 2011) as the common astrometric standard for both PACS and SPIRE because we were processing these data sets in parallel and needed a common astrometric reference frame. Moreover, the $250 \mu \mathrm{m}$ image which is our most sensitive band in the HERITAGE survey has many sources not found in the PACS bands because PACS was significantly less sensitive than SPIRE. The SPIRE $250 \mu \mathrm{m}$ catalogs have many more sources in common with the $24 \mu \mathrm{m}$ catalogs, which are also very sensitive, and thus the $24 \mu \mathrm{m}$ catalogs provides very good astrometric reference frames for HERITAGE.

For each AOR, we generated a map at $250 \mu \mathrm{m}$ and measured the positions of point sources using the IDL code Starfinder (Diolaiti et al. 2000). These positions were then compared to positions in the $24 \mu \mathrm{m}$ catalogs of the LMC (Meixner et al. 2006) and the SMC (Gordon et al. 2011) to determine the astrometry adjustments to the data. The first epoch of the LMC was only offset by a fraction of an arcsecond from the $24 \mu \mathrm{m}$ catalog in all AORs, so no adjustment was made to those data. The science demonstration phase (SDP) observations and second epoch of the LMC, and both epochs of the SMC, displayed offsets of several arcseconds that differed from AOR to AOR, so the astrometry for those AORs was adjusted to bring the average positions in line with the $24 \mu \mathrm{m}$ positions before the final map was produced. The final distribution of offsets was not welldescribed by a Gaussian, but was peaked at $1^{\prime \prime} .5$ in both galaxies, with a long tail to offsets of several arcseconds (Figure 12). We measured the offsets at the longer SPIRE wavelengths to be the same as those at $250 \mu \mathrm{m}$ to within the uncertainties implied by the larger beams; so identical corrections were applied at those wavelengths. As a final check on the astrometric correction, we extracted sources from the SPIRE 250, 350, and $500 \mu \mathrm{m}$ the combined-epoch images (Section 7 below) and did another comparison to the MIPS $24 \mu \mathrm{m}$ source locations. We found no systematic offsets which confirms that astrometry is consistent both with the MIPS $24 \mu \mathrm{m}$ and by extension the SAGE surveys, and with the PACS data sets.

\subsection{Background Subtraction, Destriping, and Masking}

The background subtraction was performed separately for each scan line. The level subtracted was defined by a linear function that fits a single data point on either side of the galaxy, each of which was the result of a sigma-clipped median after masking out the region of the galaxy. The mask was the same for all three wavelengths and was determined using a simple map at $500 \mu \mathrm{m}$, the wavelength at which the apparent extent of each galaxy was largest.

Since the observed regions were dominated by the extended emission of Magellanic ISM, we applied to the bolometer timelines the extended emission relative gain factors. These gains, determined by the SPIRE ICC, represent the ratio between the response of each bolometer to the extended emission and the average response. The difference in the response of each bolometer was mainly due to the variation of the beam area among bolometers. The use of those relative gains decreased the noise in the final maps by several tens of percent at high ( $\gtrsim$ few times $10 \mathrm{MJy} \mathrm{sr}^{-1}$ ) surface brightness.

We then applied a destriping routine to backgroundsubtracted data. This routine was a modified version of the destriping task implemented in HIPE. The routine measured the difference between the signal measured by each bolometer for each scan and the signal on the reconstructed map at the same sky coordinates. This difference, as a function of time along the scan for each bolometer, was fitted with a second-order polynomial, and subtracted from the bolometer's signal timeline. A new map was reconstructed and the procedure was repeated for 100 iterations.

Our destriping routine also included the detection of jumps in bolometer timelines and a second level deglitching. Jumps in bolometer timelines were similar to PACS long glitches (see Section 3.6), but in SPIRE they affected one bolometer at a time (since each bolometer has its own readout electronics). These jumps were detected by performing a wavelet transform with a Haar wavelet of fixed width of the difference between the signal measured by a bolometer in a scan and the signal on the reconstructed map at the same sky coordinates. If the maximum of the result was larger than a given threshold (specified as quanta of the noise), the bolometer timeline was affected by a jump and flagged to be omitted in the map making. The number of flagged timelines was $<0.5 \%$. Second level deglitching was implemented to remove glitches left over by the first level wavelet deglitcher, which worked on bolometer timelines. 

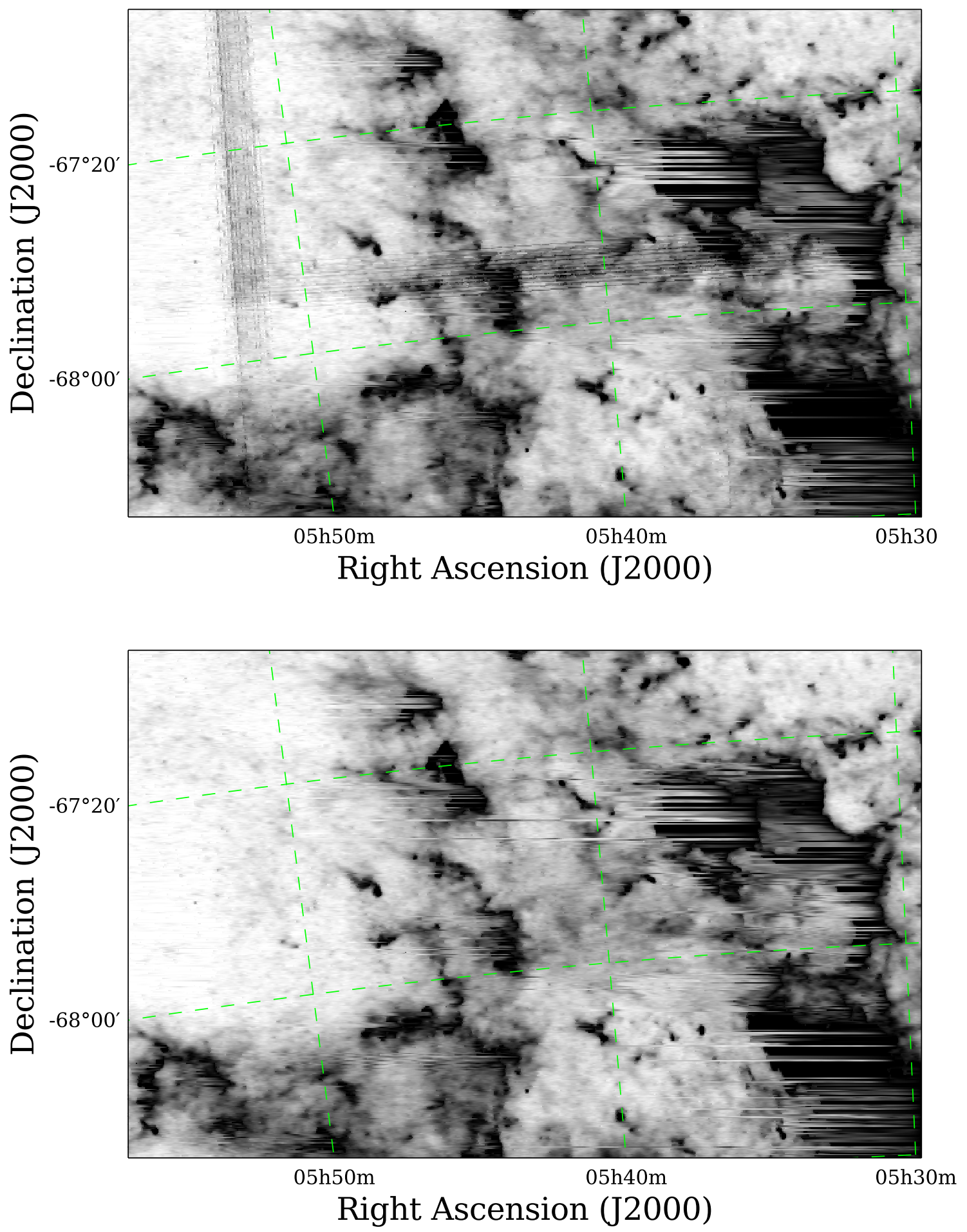

Figure 11. Top panel: a section of the SPIRE $500 \mu \mathrm{m}$ band map of the LMC produced with the standard HIPE 7 pipeline. The vertical and horizontal stripes are due to residuals left by temperature drift correction during strong temperature variations (see Section 4). Bottom panel: same region as in the top panel of the SPIRE $500 \mu \mathrm{m}$ band map produced using new temperature drift coefficients.

(A color version of this figure is available in the online journal.)

Our second level deglitching algorithm identified glitches by comparing the signal of a sample to the corresponding signal of the reconstructed map. A sample with a glitch differed from the reconstructed map by more than 5 times the map pixel's error value comprised of the instrumental noise and the standard deviation of the combined samples. This algorithm did not flag samples taken near or on bright sources. The number of flagged samples was around 10-20 per scan leg per array. The destriping routine reduced all residual drifts to less than the noise level, except for a single leg in the LMC, which we removed from subsequent processing.

\subsection{Mapping}

Map reconstruction was done with the SPIRE default mapmaking algorithm (simple map making). We converted the maps 

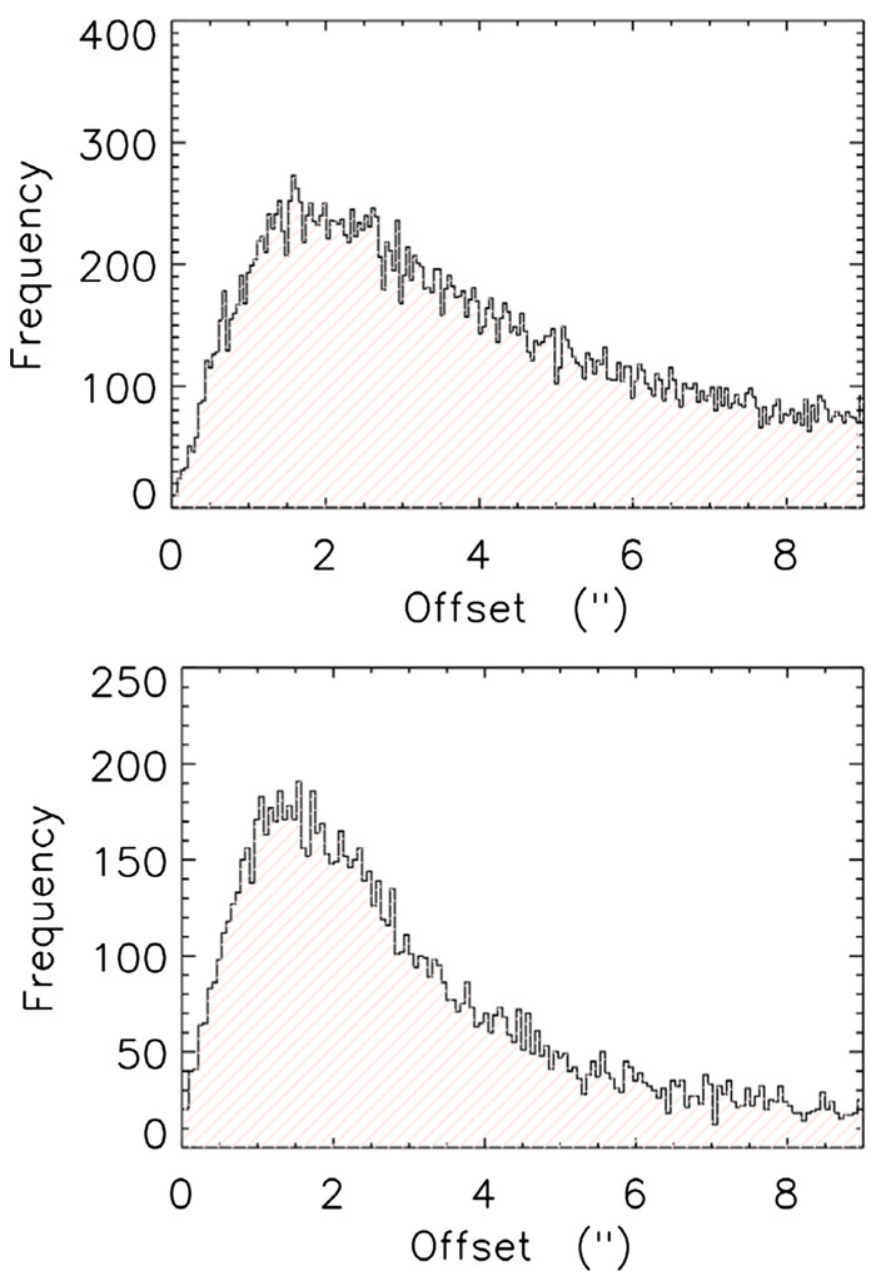

Figure 12. Top panel: the histogram of positional offsets between the SPIRE $250 \mu \mathrm{m}$ point source locations and their corresponding MIPS $24 \mu \mathrm{m}$ point source locations for the LMC. Frequency is the number of sources with a particular offset. Bottom panel: similar positional histogram offsets but for the SMC.

(A color version of this figure is available in the online journal.)

output from HIPE, which are in units of $\mathrm{Jy} \mathrm{beam}^{-1}$, to $\mathrm{MJy} \mathrm{sr}^{-1}$, by dividing by beam areas (using the revised SPIRE instrument beams posted in 2012 December) of [1.093, 1.932, 4.156] $\times$ $10^{-8}$ sr or $[465,822,1768] \operatorname{arcsec}^{2}$ at $[250,350,500] \mu \mathrm{m}$. A rough estimate of the uncertainty on the whole-galaxy measurements was determined by comparing the total flux density of each galaxy to interpolations between the prior submillimeter data of the LMC and SMC compiled by Aguirre et al. (2003). The SPIRE data we present here were found to be consistent within the errors for both the LMC and SMC, respectively. In addition to the two SPIRE epochs, we incorporated the $18 \mathrm{hr}$ of SDP data, which covered $2^{\circ} \times 8^{\circ}$ strip through the LMC center, at a position angle of $\sim 22.5$, described by Meixner et al. (2010) into the final LMC SPIRE map but not for the final PACS map because of the bad striping of the PACS SDP data. The limiting surface brightnesses listed in Table 3 represent $10 \sigma$ of the median value of the noise maps produced during the SPIRE data processing.

\section{SPATIAL DISTRIBUTION OF LMC AND SMC HERSCHEL EMISSION}

The images resulting from the HERITAGE project provide the highest spatial resolution view of the emission at these wavelengths to date. Figure 13 shows the HERITAGE data in all five bands for the LMC with the MIPS $70 \mu \mathrm{m}$ image from SAGE-LMC (Meixner et al. 2006). At first glance, all the images look identical, which indicates that the emission from all these bands arises from the same sources of ISM dust. The images show a disk of dust emission with complex filamentary structure punctuated by bright compact regions. The brightest of these compact regions, located on the eastern side, slightly south of center, are the highly active star formation regions of 30 Doradus and N160 slightly further south. The other bright regions are also associated with active star formation where the bright young OB stars efficiently heat the ISM dust. On closer inspection, one notices that the contrast between these bright regions and the fainter diffuse ISM emission decreases with increasing wavelength. This contrast becomes more apparent in the three-color image of the PACS 100 and $160 \mu \mathrm{m}$ data and the SPIRE $250 \mu \mathrm{m}$ data (Figure 14). If all the images had an identical morphology on all scales, the images would appear as a gray scale. The color differences apparent in the image reflect the differences in temperature of the dust emission. The bright white spots are the hottest regions, the blue or green regions are warm, and the red regions are coolest.

The HERITAGE data for the SMC reveal a more disturbed structure with a NE-SW bar to the west and an extension to the east southeast that is called the "wing" of the SMC (Figure 15). The similarity of the PACS and SPIRE band emissions underlies the common source of ISM dust emission. West beyond the wing is the beginning of the Magellanic Band between the LMC and SMC, the so-called Tail of the SMC, and very little emission is apparent in this region. A low level of wispy emission is apparent throughout the SMC field of view and the majority of this wispy emission is attributable to the MW's IR cirrus emission (e.g., Gordon et al. 2009). Part of the reason for the lower emission in the "Tail" is undoubtedly due to a decrease in dust-to-gas mass ratio in the tail compared to the wing and bar of the SMC (Gordon et al. 2009). The SMC ISM dust emission also has a filamentary appearance with bright knots of emission, especially in the bar, such as N66 in the northeast part of the bar. The three-color image (Figure 16) highlights the bright knots in white as the hottest dust regions. The Herschel spatial resolution is sufficient to reveal the mini-spiral shape of N66 in the northeast part of the bar. The three-color image of the SMC also reveals a range of hot, warm, and cool dust regions within the galaxy (Figure 16).

Figures 17 and 18 compare the spatial distribution of the Herschel emission in the LMC and SMC to other galaxy-wide tracers of the stars and gas. The Spitzer IRAC $3.6 \mu \mathrm{m}$ band traces the evolved stellar population, which have SED peaks in the near-IR (Meixner et al. 2006; Gordon et al. 2011). The SHASSA $\mathrm{H} \alpha$ image traces the ionized atomic hydrogen gas (Gaustad et al. 2001). The ATCA/Parkes H I $21 \mathrm{~cm}$ image traces the neutral atomic hydrogen gas in the LMC (Kim et al. 2003) and SMC (Stanimirović et al. 1999). The NANTEN CO $J=1-0$ emission traces molecular gas in the LMC (Fukui et al. 2008) and SMC (Mizuno et al. 2001).

The distribution of the Herschel emission does not resemble that of the evolved stellar population, the source of the stellar wind and dust production, which is traced by the IRAC $3.6 \mu \mathrm{m}$ band. Indeed the LMC bar, which is dominated by light from the evolved stars, is not apparent in the Herschel images. Herschel emission has a similar morphology to ISM gas tracers, especially $\mathrm{H}$, indicating that Herschel emission arises primarily from the cool dust of the ISM. However, the dynamic range of the 


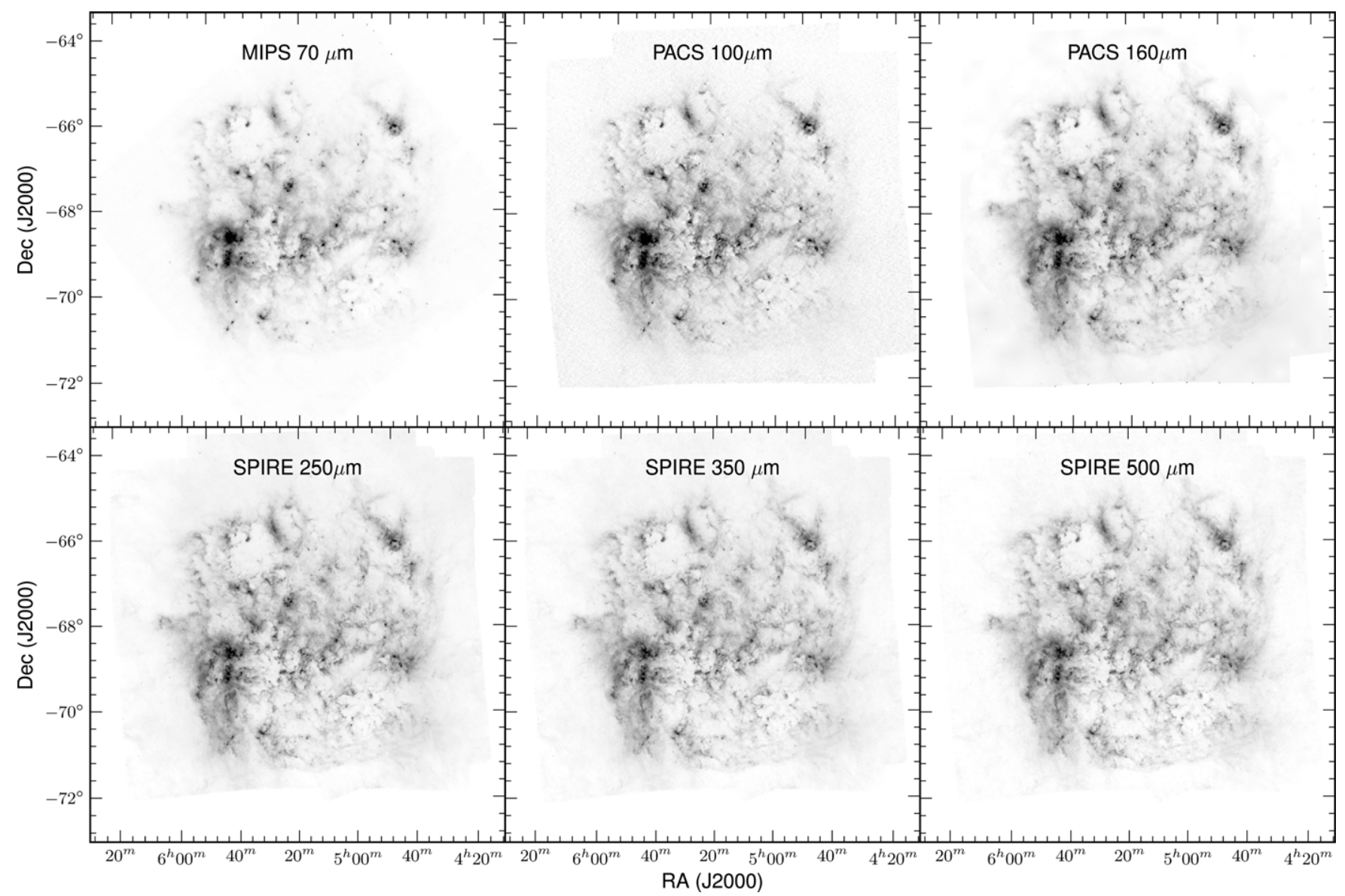

Figure 13. HERITAGE data for the LMC with the Spitzer-SAGE-LMC MIPS $70 \mu \mathrm{m}$ image (Meixner et al. 2006) for comparison. Together these data sets sample the spectral energy distribution of ISM dust emission. The gray-scale displays are all in arcsinh with the following ranges: MIPS $70 \mu \mathrm{m}, 0-400 \mathrm{MJy} \mathrm{sr}^{-1}$; PACS $100 \mu \mathrm{m}$, $0-400 \mathrm{MJy} \mathrm{sr}^{-1}$; PACS $160 \mu \mathrm{m}, 5-500 \mathrm{MJy} \mathrm{sr}^{-1}$; SPIRE $250 \mu \mathrm{m}, 1-300 \mathrm{MJy} \mathrm{s}^{-1}$; SPIRE $350 \mu \mathrm{m}, 0-170 \mathrm{MJy} \mathrm{sr}^{-1}$; SPIRE $500 \mu \mathrm{m}, 0-70 \mathrm{MJy} \mathrm{sr}^{-1}$.

Herschel images is larger than that of the $\mathrm{H}$ i because the dust emission is very sensitive to temperature. The bright, hotter dust regions in the Herschel images appear morphologically in the same places as the brightest $\mathrm{H} \alpha$ emission in the SHASSA images. For example, the brightest Hir regions in the SMC, N66, and in the LMC, 30 Doradus, appear as bright regions of emission in the PACS $100 \mu \mathrm{m}$ and SPIRE $250 \mu \mathrm{m}$ bands. Massive OB stars that ionize gas are also the most effective at heating the dust, explaining this morphological correspondence. Skibba et al. (2012) presents a detailed comparison of the stellar and dust luminosity.

The CO $J=1-0$ emission bears little overall resemblance to the Herschel bands because most of the ISM gas in the LMC and $\mathrm{SMC}$ is neutral atomic gas. However, if we look at the structures detected by the CO $J=1-0$ emission and search for them in the Herschel images, we do find enhanced emission. The ISM gas regions detected in $\mathrm{CO}$ are associated with giant molecular cloud complexes (e.g., Kawamura et al. 2009) and thus with larger column densities of ISM gas and emission, explaining this correlation (cf., Bernard et al. 2008).

Therefore the Herschel emission is affected by both the temperature and column mass density of the ISM dust. We have presented analyses of the ISM dust mass using the LMC SDP strip in Meixner et al. (2010), Gordon et al. (2010), and Galliano et al. (2011). Analysis of ISM dust mass and temperature based on the Herschel emission from the whole LMC and SMC will be covered in a forthcoming paper (K. D. Gordon et al. in preparation). Analyses of the dust-to-gas mass ratio in the
ISM on the LMC SDP strip were presented by Meixner et al. (2010) and Roman-Duval et al. (2010). Complete analysis of the dust-to-gas mass ratio across the whole LMC and SMC by comparing the Herschel data with gas tracers will be presented by J. Roman-Duval et al. (in preparation).

\section{GLOBAL SPECTRAL ENERGY DISTRIBUTIONS OF THE LMC AND SMC}

We calculated the total galaxy flux in the LMC and SMC by summing over the fluxes of the background-subtracted images. We then adopted a signal-to-noise $(\mathrm{S} / \mathrm{N})$ cut for the pixels incorporated into the flux summation, which primarily improved the PACS $100 \mu \mathrm{m}$ flux for the SMC. Table 4 lists the total galaxy fluxes for the LMC and SMC for all five bands of the HERITAGE survey. We compared these HERITAGE fluxes, shown as red triangles, to prior measurements, which are shown in blue circles, in the SED in Figure 19. The fluxes for both galaxies measured in prior work are taken from the recent compilation of measurements from UV to radio wavelengths including measurements from a combination of ground-based telescopes (e.g., at optical and near-IR wavelengths) and spacebased missions such as IRAS, the Infrared Space Observatory, and Spitzer (Israel et al. 2010). For the SMC, we also include the SAGE-SMC MIPS measurements (Gordon et al. 2011). In order to investigate the submillimeter excess emission in the Magellanic Clouds, Israel et al. (2010) measured the total fluxes of the LMC and SMC from two all sky CMB satellites: 


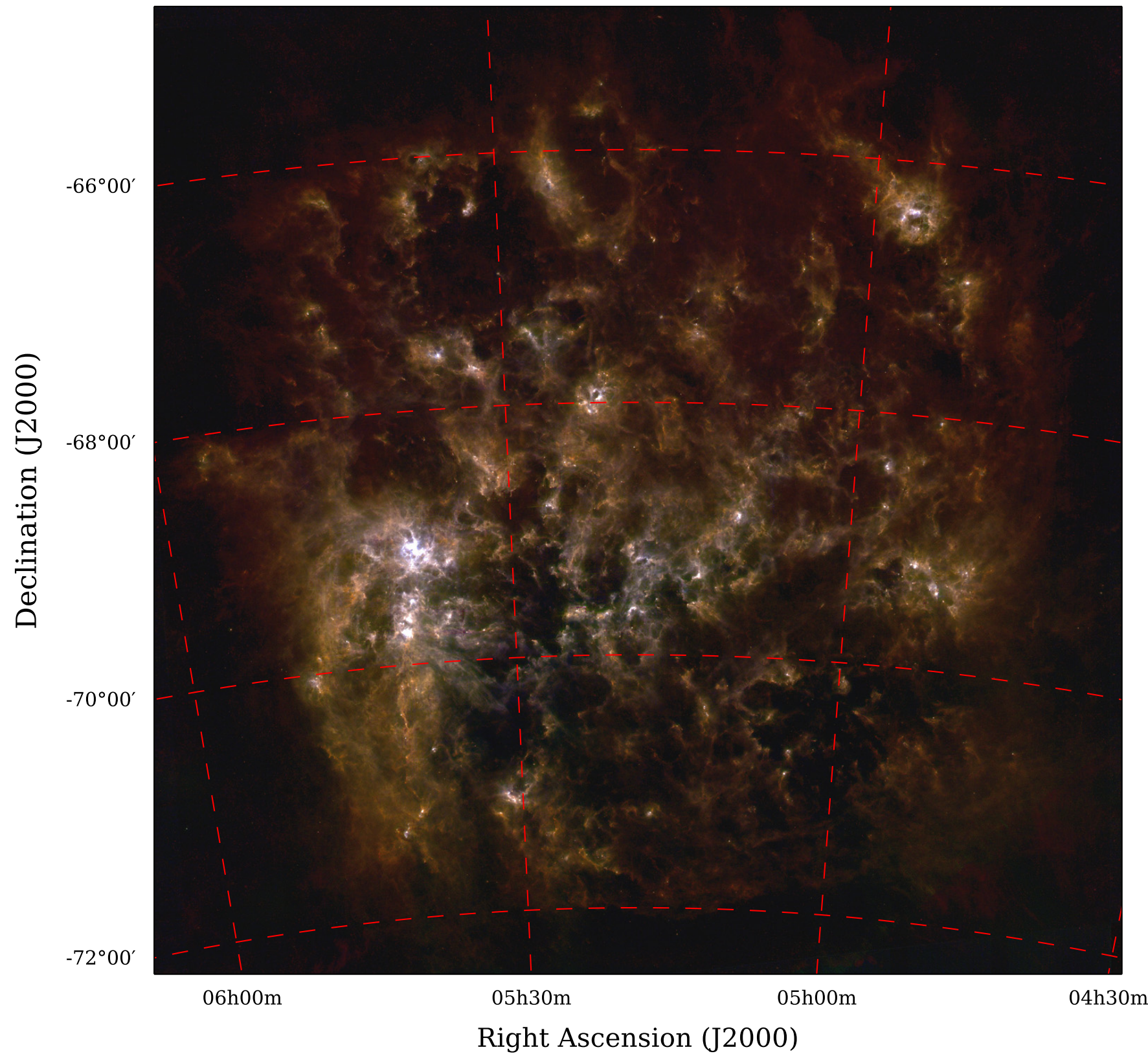

Figure 14. LMC HERITAGE data. Red corresponds to SPIRE $250 \mu \mathrm{m}$, green to PACS $160 \mu \mathrm{m}$, and blue to PACS $100 \mu \mathrm{m}$.

Table 4

Total Galaxy Fluxes in SED

\begin{tabular}{|c|c|c|c|c|c|}
\hline \multirow[t]{2}{*}{ Parameter } & \multicolumn{2}{|c|}{$\operatorname{PACS} \lambda(\mu \mathrm{m})$} & \multicolumn{3}{|c|}{ SPIRE $\lambda(\mu \mathrm{m})$} \\
\hline & 100 & 160 & 250 & 350 & 500 \\
\hline LMC flux (Jy) & $1.6 \pm 0.2 \times 10^{5}$ & $2.7 \pm 0.4 \times 10^{5}$ & $1.4 \pm 0.1 \times 10^{5}$ & $6.9 \pm 0.7 \times 10^{4}$ & $3.0 \pm 0.3 \times 10^{4}$ \\
\hline SMC flux (Jy) & $1.9 \pm 0.3 \times 10^{4}$ & $3.0 \pm 0.4 \times 10^{4}$ & $1.0 \pm 0.1 \times 10^{4}$ & $5.9 \pm 0.6 \times 10^{3}$ & $2.9 \pm 0.3 \times 10^{3}$ \\
\hline
\end{tabular}

COBE (Boggess et al. 1992) and the Wilkinson Microwave Anisotropy Probe (WMAP; Bennett et al. 2003a, 2003b). For the $C O B E$ satellite, they used data from the DIRBE instrument (Silverberg et al. 1993) and the FIRAS instrument (Fixsen et al. 1994; Wright et al. 1991). On this topic of the submillimeter excess, the Planck Collaboration et al. (2011) reported the flux measurements for the LMC and SMC at Planck wavelengths for the total flux, the CMB subtracted flux and the CMB+MW foreground subtracted flux. By analyzing these different fluxes, they claim that the submillimeter excess in these galaxies may well have a significant contribution from CMB fluctuation emission and in the case of the LMC it may explain all of the excess emission at wavelengths much longer than probed by Herschel. In HERITAGE we probe a different type of excess emission from the ISM dust (e.g., Gordon et al. 2010). Our preliminary analysis of the submillimeter dust emission measured by HERITAGE suggests the $500 \mu \mathrm{m}$ excess emission regions are correlated with LMC ISM structures so likely 


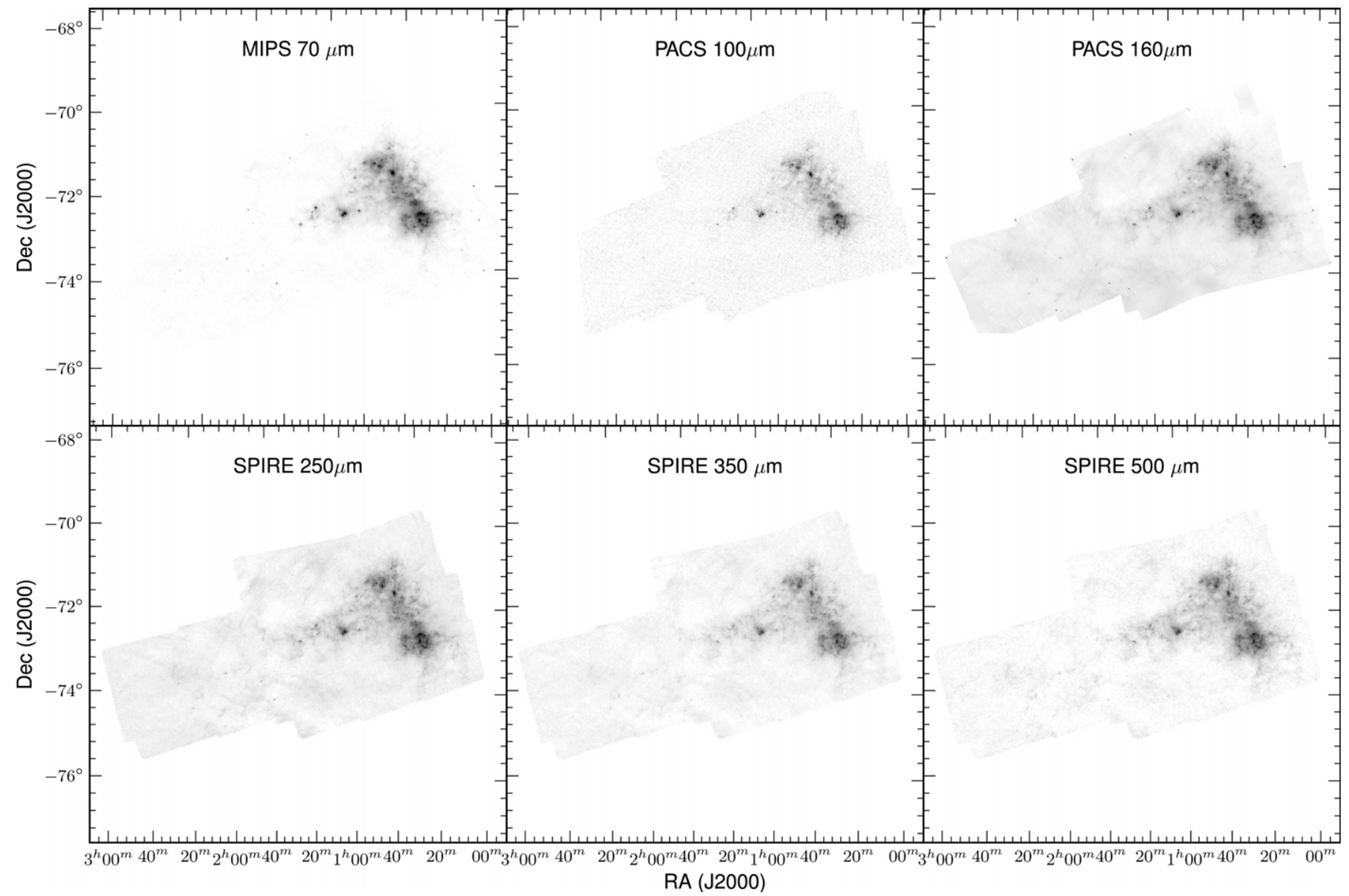

Figure 15. HERITAGE data for the SMC with the Spitzer-SAGE-SMC MIPS $70 \mu \mathrm{m}$ image (Gordon et al. 2011) for comparison. Together these data sets sample the spectral energy distribution of ISM dust emission. The gray-scale displays are all in arcsinh with the following ranges: MIPS $70 \mu \mathrm{m}, 0-100 \mathrm{MJy} \mathrm{sr}^{-1}$; PACS

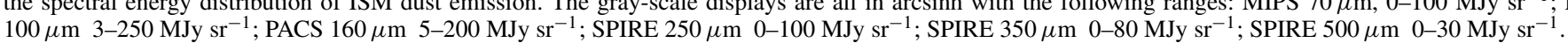

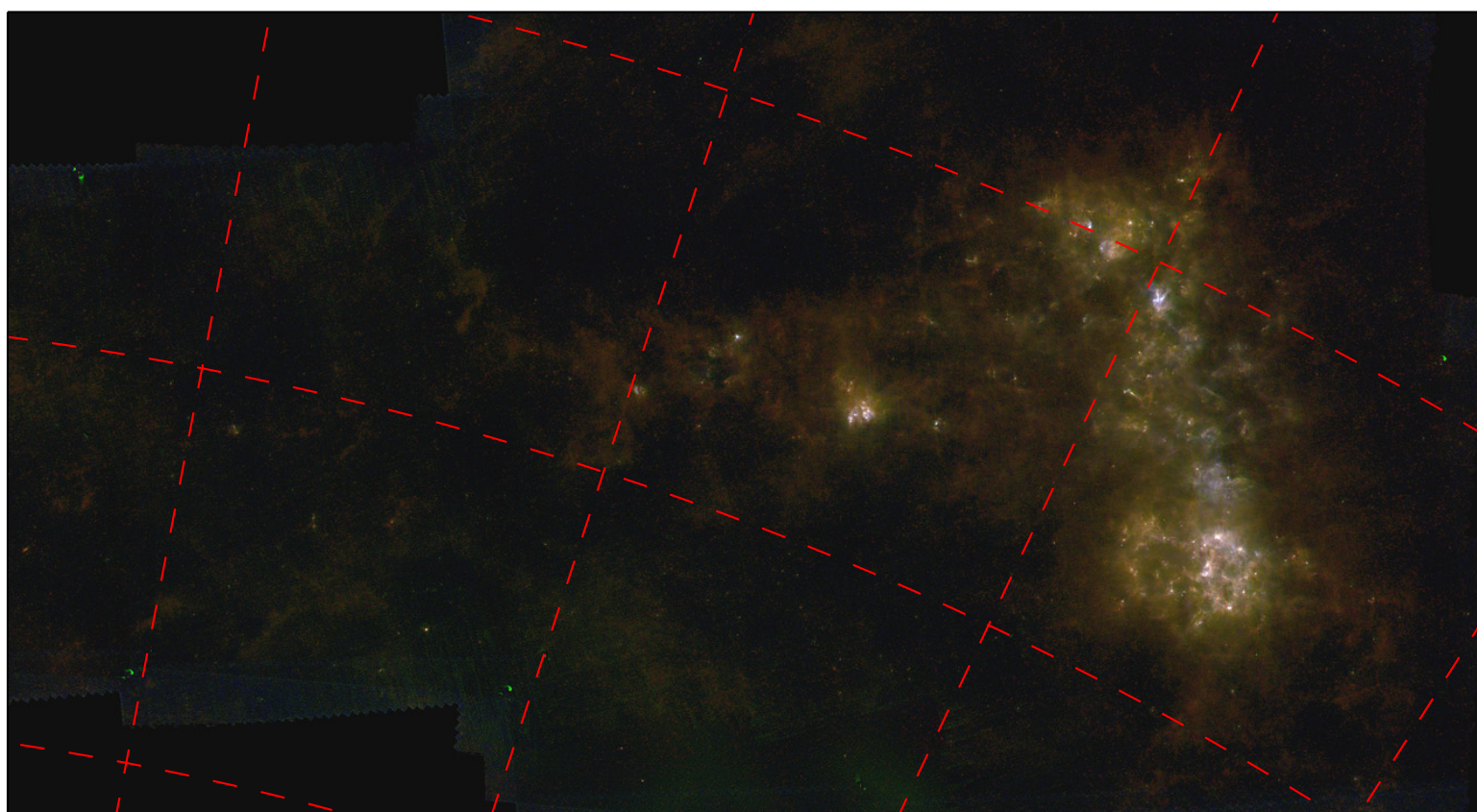

$02 \mathrm{~h} 00 \mathrm{~m}$

$01 \mathrm{~h} 30 \mathrm{~m}$

$01 \mathrm{~h} 00 \mathrm{~m}$

$00 \mathrm{~h} 30 \mathrm{~m}$

Right Ascension (J2000)

Figure 16. SMC HERITAGE data. Red corresponds to SPIRE $250 \mu \mathrm{m}$, green to PACS $160 \mu \mathrm{m}$, and blue to PACS $100 \mu \mathrm{m}$. 


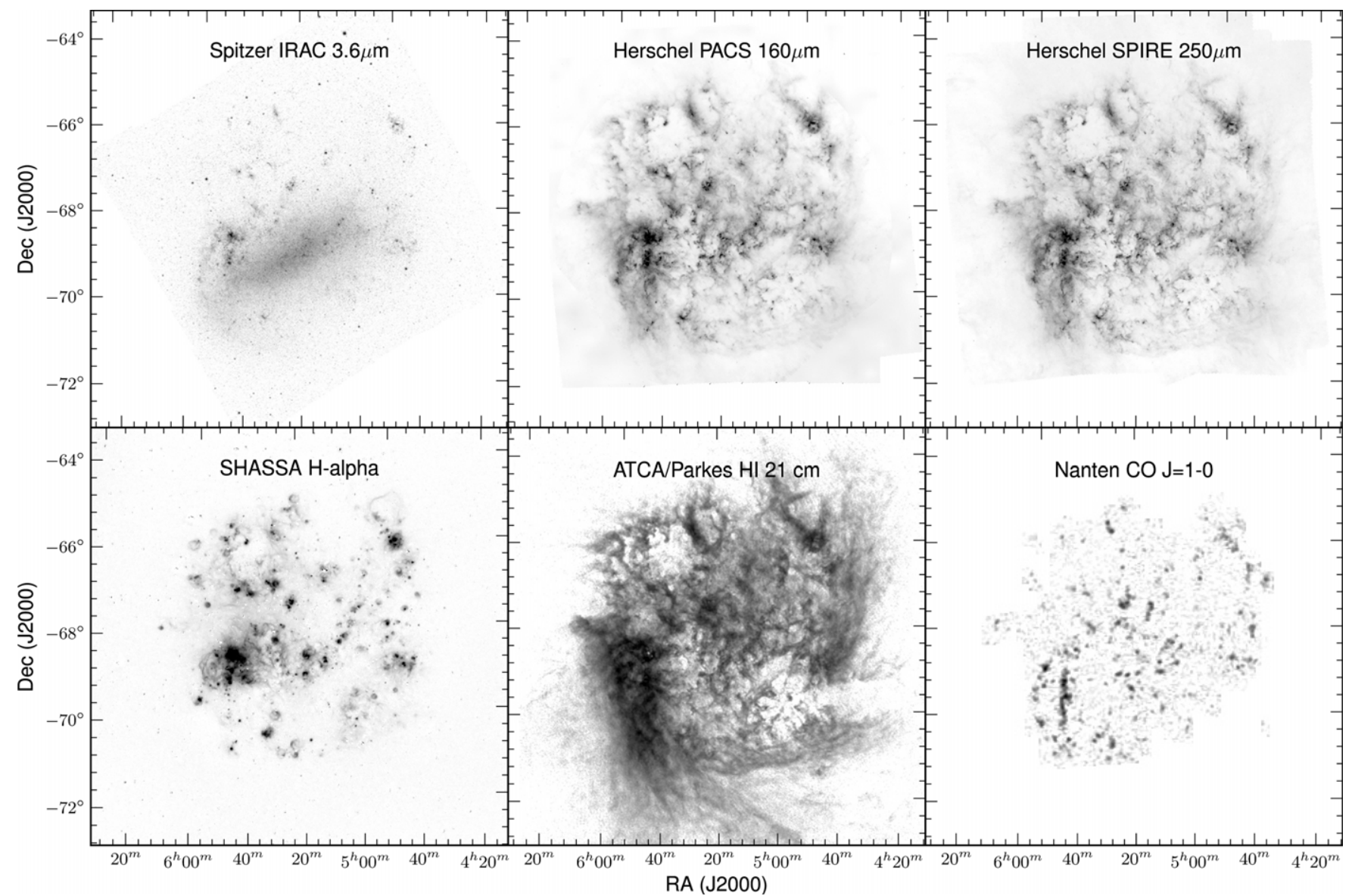

Figure 17. LMC survey data. From top to bottom, left to right: the Spitzer IRAC $3.6 \mu$ m image from the SAGE-LMC survey (Meixner et al. 2006) reveals the old stellar population, which is ejecting dust into the ISM. The Herschel PACS $160 \mu \mathrm{m}$ and SPIRE $250 \mu \mathrm{m}$ image from the HERITAGE survey reveal the coolest dust emission, which is dominated by the larger dust grains. The $\mathrm{H} \alpha$ emission from SHASSA (Gaustad et al. 2001) shows the distribution of the diffuse ionized gas, punctuated by H II regions. The H I $21 \mathrm{~cm}$ emission (Kim et al. 2003) show the location of the atomic neutral hydrogen. The CO $J=1-0$ emission survey by NANTEN (Fukui et al. 2008) traces the highest column density of molecular gas. The gray scale displays are all in arcsinh with the following ranges: IRAC $3.6 \mu \mathrm{m} 0-5 \mathrm{MJy} \mathrm{sr}^{-1}$; PACS

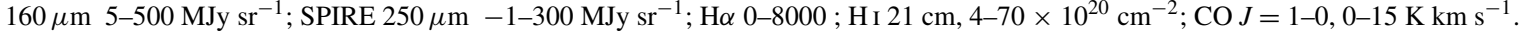

originate from dust emission in the galaxies (e.g., Galliano et al. 2011; Verdugo et al. 2013). Further analysis of this $500 \mu \mathrm{m}$ excess emission across the LMC and SMC will be discussed in a future paper (K. D. Gordon et al. in preparation).

In Figure 19, we plot the total LMC and SMC fluxes without any $\mathrm{CMB}$ subtraction measured by the Planck Collaboration et al. (2011) in order to be consistent with our HERITAGE fluxes and the prior work. We calculate the Planck total fluxes by using the reported average surface brightness times the circular area defined by a radius listed in the Table 2 caption from Planck Collaboration et al. (2011). The Planck data, which are shown in green squares in Figure 19, appear in reasonable agreement with the prior work that includes the WMAP and $C O B E / D I R B E / F I R A S$ results. In comparison to this complete SED of the LMC and SMC, the HERITAGE SPIRE photometry data agrees within the respective errors of the data sets. The HERITAGE PACS $100 \mu \mathrm{m}$ data appears to also agree with prior work within the errorbars. The HERITAGE PACS $160 \mu \mathrm{m}$ flux point appears consistent with prior measurements for the LMC but appears to slightly exceed the known photometry for the SMC (Gordon et al. 2011; Leroy et al. 2007).

\section{HERITAGE POINT SOURCE EXTRACTIONS}

The variable background emission present in the HERITAGE mosaics presents a challenging environment in which to attempt point-source photometry. After experimenting with several source extraction packages, we chose the PSF-fitting software Starfinder (Diolaiti et al. 2000; see Section 7.3). Aperture photometry is not feasible for automated production of catalogs because the diffuse emission is too complex for automated annulus selections to calculate background levels. Starfinder uses a smoothing algorithm to estimate the local background and then iterates on the background as sources are found and extracted (Bertin \& Arnouts 1996).

The HERITAGE catalogs were produced through an iterative process. The first step was to find and extract sources with Starfinder, using PSFs provided by the Herschel Science Center. ${ }^{38}$ The detection threshold was set at $5 \sigma$ above the noise level. The correlation parameter, a measure of goodness of fit (where 1 is perfect), was used to remove sources with values below 0.75 . Source lists were made from not only the combinedepochs image but also each individual epoch image (two epochs for SPIRE LMC and SMC data and PACS LMC data, and three epochs for the PACS SMC data). These additional single epoch sourcelists were used to improve the reliability of the final catalog of sources, which contains only sources detected in the combined-epoch images. For each of the five wavelengths of data (PACS $100 \mu \mathrm{m}$ and $160 \mu \mathrm{m}$ and SPIRE $250 \mu \mathrm{m}, 350 \mu \mathrm{m}$,

\footnotetext{
38 http://herschel.esac.esa.int/twiki/bin/view/Public/PacsCalibrationWeb/ and
} http://herschel.esac.esa.int/twiki/bin/view/Public/SpireCalibrationWeb/ 


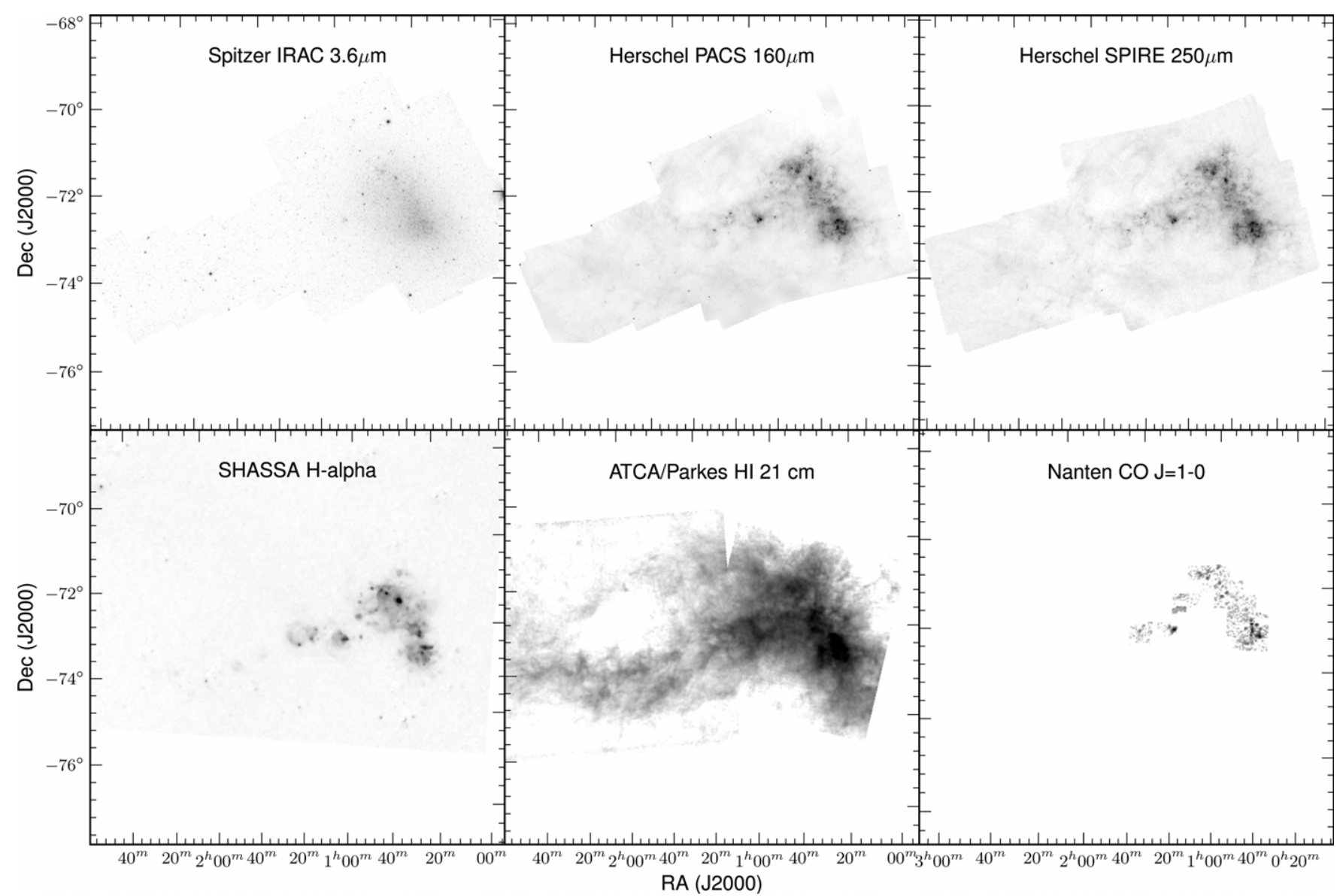

Figure 18. Same type of survey data as in Figure 17 but from different sources, for the SMC. The Spitzer IRAC $3.6 \mu$ m image from the SAGE-SMC survey (Gordon et al. 2011) reveals the old stellar population. The Herschel PACS $160 \mu \mathrm{m}$ and SPIRE $250 \mu \mathrm{m}$ images from the HERITAGE survey reveal the coolest dust emission. The $\mathrm{H} \alpha$ emission from SHASSA (Gaustad et al. 2001) shows the distribution of the diffuse ionized gas and H II regions. The H I $21 \mathrm{~cm}$ emission (Stanimirovic et al. 1999; Muller et al. 2003a) show the location of the atomic neutral hydrogen. The CO $J=1-0$ emission survey by NANTEN (Mizuno et al. 2001) traces the molecular gas. The gray scale displays are all in arcsinh with the following ranges: IRAC $3.6 \mu \mathrm{m}, 0-5 \mathrm{MJy} \mathrm{sr}^{-1} ;$ PACS $160 \mu \mathrm{m}, 2-200 \mathrm{MJy} \mathrm{sr}^{-1} ;$ SPIRE $250 \mu \mathrm{m}$, $0-100 \mathrm{MJy} \mathrm{sr}^{-1} ; \mathrm{H} \alpha-10-5000 ; \mathrm{H} \mathrm{I} 21 \mathrm{~cm}, 5-100 \times 10^{20} \mathrm{~cm}^{-2}$; CO $J=1-0,0-2.2 \mathrm{~K} \mathrm{~km} \mathrm{~s}^{-1}$.

and $500 \mu \mathrm{m})$ the individual epoch source lists and combinedepoch image source list were matched using a simple nearest match criteria of $0.7 \times$ FWHM. The subset of matched sources for each band was then identified for enhanced study to determine source reliability and photometric accuracy. For these subsets we fit a 2D Gaussian to each source. We then applied an additional cut to our subset of sources, selecting only the sources that produced a measured FWHM in both $x$ and $y$ directions without extreme values (between $1 / 3$ and 3 times the Herschel FWHM value). A histogram of those FWHM values for each wavelength band was then used, as well as the mean and median values to determine an optimal FWHM for the extraction PSF. The Herschel Science Center PSFs were then broadened with a Gaussian of appropriate width to produce a new PSF with the desired FWHM (see Figure 20). Many factors may have contributed to make the HERITAGE PSFs more broad than the Herschel PSFs. The HERITAGE mosaics were created from lengthy scanning observations, in which pointing uncertainties, changing position angle, and multiple observations can result in a broadened PSF. Unfortunately, the degree of broadening was variable across images. The PSF from the combined image of all the epochs was more broad and circular than those from the individual epochs.

Using these broadened PSFs, Starfinder was re-run on the HERITAGE images. An iterative process was employed. The first pass of Starfinder extracted the vast majority of sources.
Then the residual image (the image after Starfinder removes the found sources) was resubmitted to Starfinder a second time to find additional sources that may have been lost in the wings of the PSF of bright sources. Additionally, after each Starfinder iteration, we used a tweaking algorithm on the residual images to correct for over-extracted sources in high/complex background areas. This algorithm is described in more detail in the Spitzer GLIMPSE processing documents. ${ }^{39}$ This additional tweaking step limits the occurrence of multiple source extraction from an overly broad source with a PSF smaller than the FWHM of the broad source.

\subsection{Flux Uncertainties}

Starfinder produces an uncertainty associated with each extracted source, which is determined by the mode in which Starfinder is run. For the SPIRE data we used the associated error maps produced from the image mosaicking process. For the PACS images we allowed Starfinder to determine the uncertainty from the rms in the background part of the image. The PACS images contain a degree of low level striping that adds more uncertainty to the lower background level sources. However, the uncertainty that Starfinder determines is only one component of several that contribute to the overall uncertainty of the fluxes in the HERITAGE catalogs. These additional components include:

\footnotetext{
39 http://www.astro.wisc.edu/glimpse/glimpse_photometry_v1.0.pdf
} 

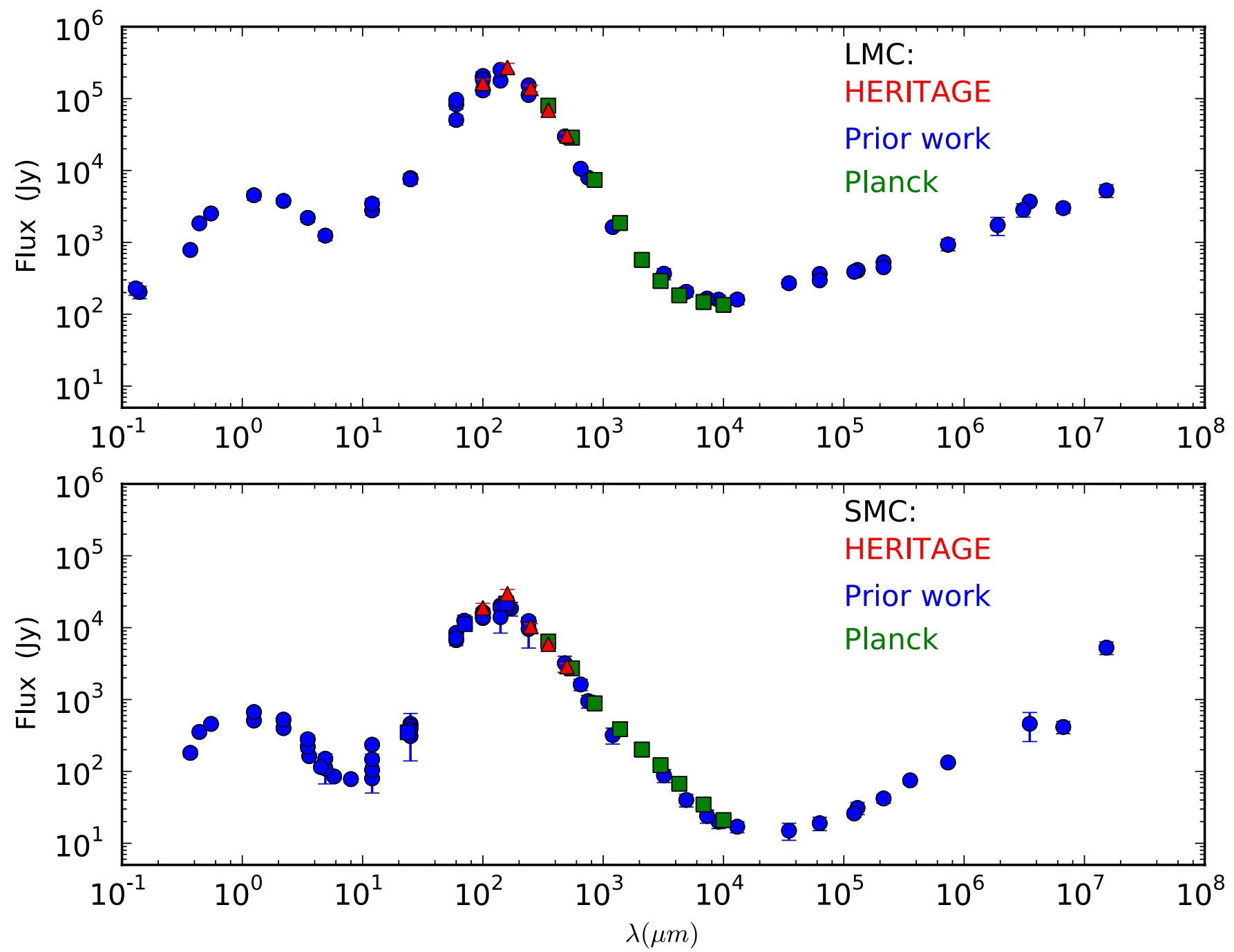

Figure 19. Spectral energy distributions of the whole LMC and SMC cover the UV to radio wavelengths. Prior work summarized by Israel et al. (2010) includes data from ground and space based missions such as IRAS, DIRBE, TopHat and WMAP. For the SMC, we also include prior work (blue squares) from Gordon et al. (2011). The HERITAGE photometry measured in the PACS and SPIRE bands are shown in red. The Planck measurements are shown in green (Planck Collaboration et al. 2011).

(A color version of this figure is available in the online journal.)

determination of the background, the accuracy of the PSF used in the extraction, and the degree to which the extracted source is a true point source.

These other components often dominate in the HERITAGE data. We attempt to quantify these components and give more generalized uncertainties to the HERITAGE source lists by analyzing the flux measurements of many sources observed in two different epochs. Ideally, observing a single source many times will provide an accurate estimate of the uncertainties. We observed each source only two times, typically, but we can compare hundreds of different sources of similar brightness and background levels. Thus, we matched sources from the combinedepoch images to the epoch 1 and 2 images, grouped sources of similar brightness and background levels, and compared their fluxes using the formalization found in the SAGE-LMC Data Delivery document (Section 3.5) for their parameter $V^{40}$ :

$$
V=\frac{F_{1}-F_{2}}{\left(\sigma_{1}^{2}+\sigma_{2}^{2}\right)^{1 / 2}},
$$

\footnotetext{
$\overline{40}$ See http://sage.stsci.edu/SAGE-LMC-deliv2.pdf.
}

where $F_{1}$ is the epoch 1 flux of a source, $F_{2}$ is the epoch 2 flux of the same source, and $\sigma_{1}$ and $\sigma_{2}$ are the uncertainty estimates of the epoch 1 and epoch 2 fluxes. The uncertainties are calculated as follows, using $\sigma_{1}$ as the example:

$$
\begin{aligned}
& \sigma_{1}=\left(s_{1}^{2}+s_{1 f}^{2}+s_{1 s}^{2}\right)^{1 / 2} \\
& s_{1}=\text { unc }_{1} * u_{\text {cor }} \\
& s_{1 f}=F_{1} * f_{\text {cor }} \\
& s_{1 s}=\operatorname{sky}_{1} * s_{\text {cor }},
\end{aligned}
$$

where unc ${ }_{1}$ is the output uncertainty from Starfinder. We include additional uncertainties $s_{1 f}$ and $s_{1 s}$ that depend on the flux and sky values (with coefficients $f_{\text {cor }}$ and $s_{\text {cor }}$ respectively). We also include an additional coefficient $u_{\text {cor }}$ on $s_{1}$ to account for additional low flux level uncertainty that the Starfinder uncertainty underestimates. Values of $u_{\text {cor }}, f_{\text {cor }}$ and $s_{\text {cor }}$ are 
PACS100 Combined Epoch image

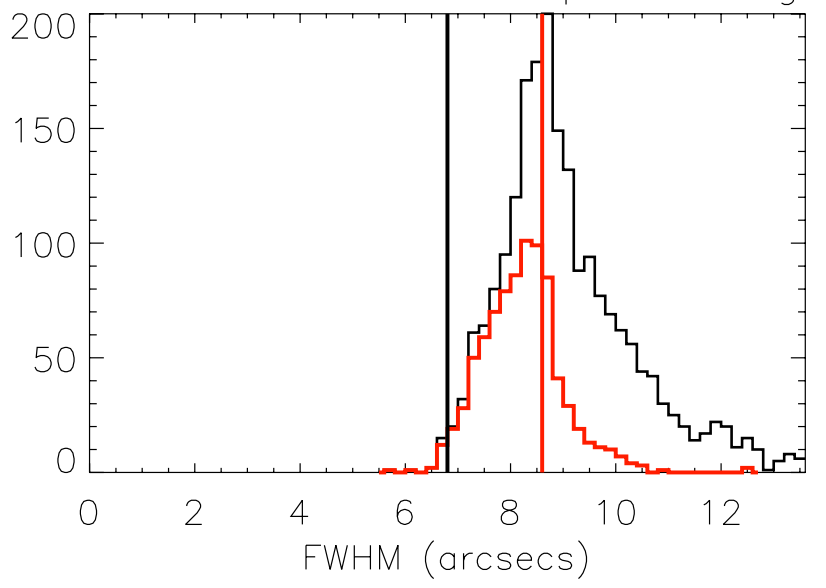

SPIRE250 Combined Epoch image

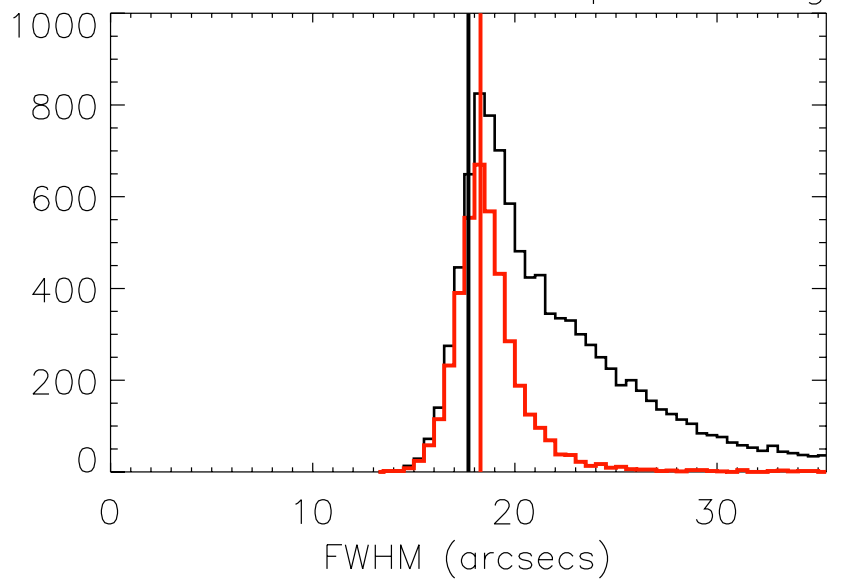

SPIRE500 Combined Epoch image

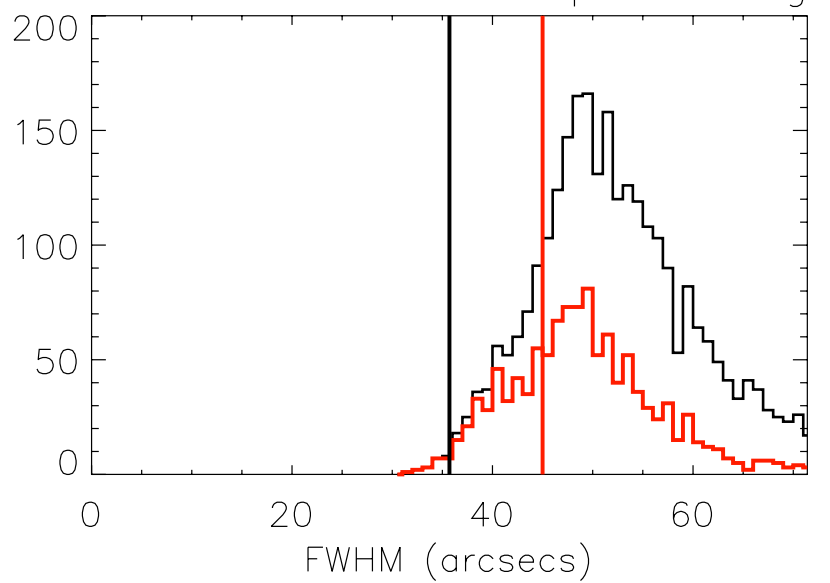

PACS160 Combined Epoch image

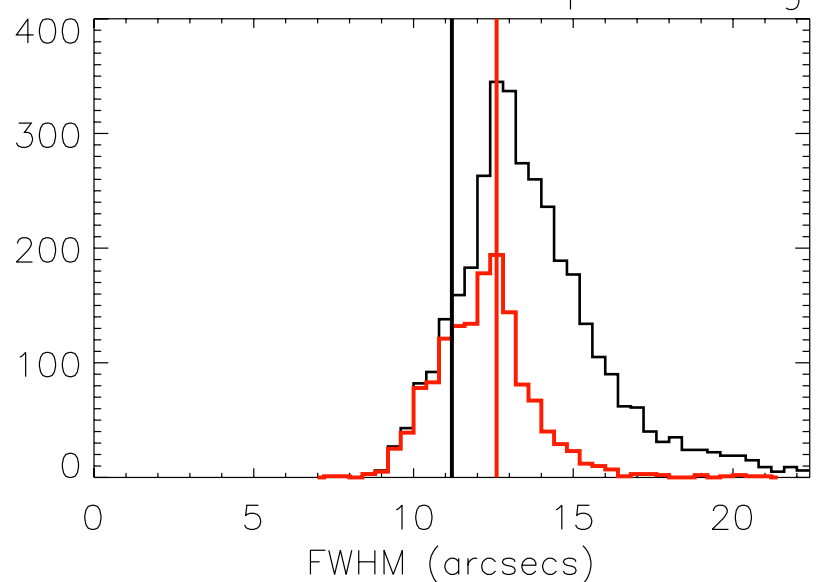

SPIRE350 Combined Epoch image

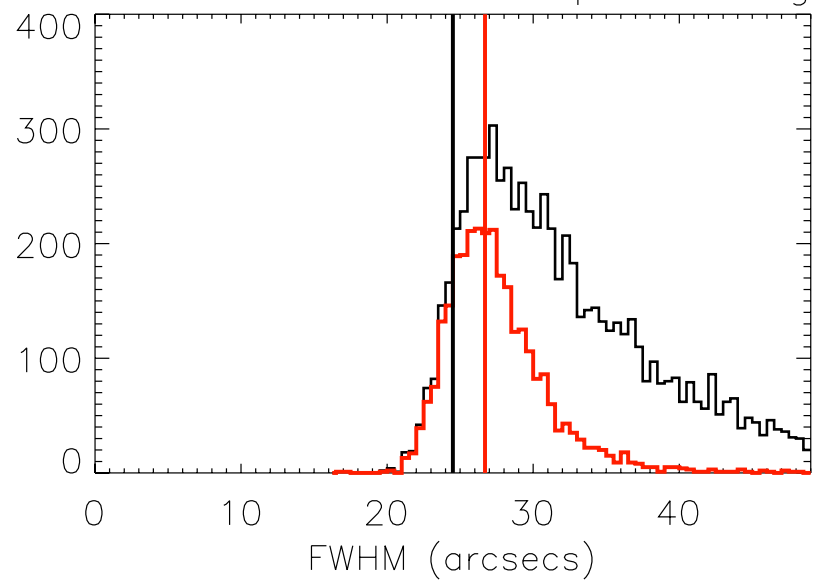

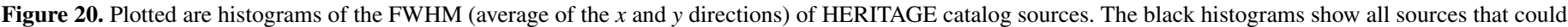

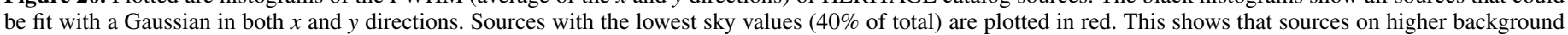

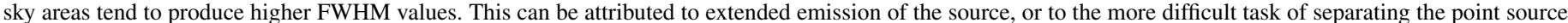

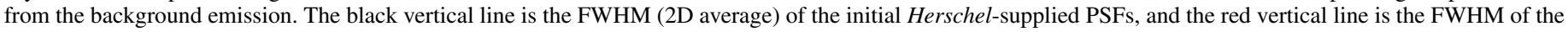
broadened PSFs that were used to produce the HERITAGE catalogs. The SPIRE $500 \mu \mathrm{m}$ PSF values suffer the most from background sky contamination.

(A color version of this figure is available in the online journal.)

empirically determined to optimize the resultant uncertainties so that the distribution of flux differences $V$ between epoch 1 and epoch 2, for sources in similar groupings, forms a Gaussian distribution with $\sigma=1$ (see Figure 21). Note that the center position of 0 in the histograms shows that there are no systematic offsets in the fluxes between epochs 1 and 2 . The values of $u_{\text {cor }}$, $f_{\text {cor }}$ and $s_{\text {cor }}$ vary with wavelength and each galaxy (LMC and SMC); for example the LMC SPIRE $250 \mu \mathrm{m}$ single epoch values are $u_{\text {cor }}=1.4, f_{\text {cor }}=0.06, s_{\text {cor }}=0.35$. Table 5 lists the final values for both the LMC and SMC datasets. 


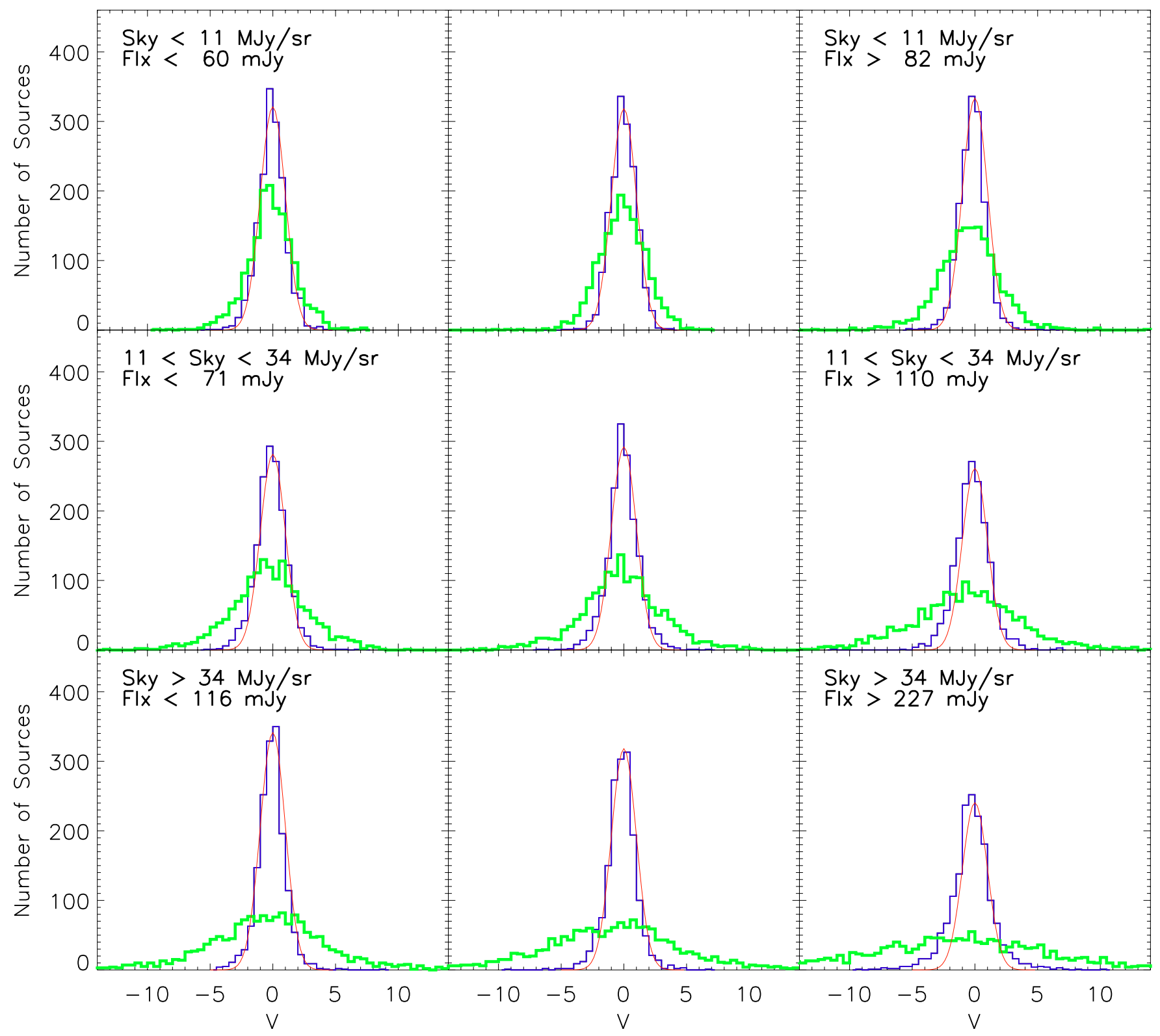

Figure 21. Each panel shows a subset of sources from the SPIRE $250 \mu \mathrm{m}$ catalog plotted as histograms of the variability parameter $V$ (Equation (1)). Each panel has an equal number of sources but has distinct ranges of sky and source fluxes, which are labeled on the left and right panels and are intermediate in values for the middle panels. The flux ranges increase from left to right, and the sky values increase from top to bottom. The red, thin curve shows the expected histogram width if the distribution of $V \mathrm{~s}$ has $\sigma=1$. A histogram broader than the red curve implies uncertainties are too small compared to the difference between flux values (assuming minimal number of variable sources). The green, thickest line histograms show the values based on Starfinder uncertainties only. The width of the green histogram increases for both increased sky and flux values indicating larger differences, and therefore larger uncertainties, in the epoch 1 and 2 flux measurements. The blue, thinner line histograms show the values based on the adjusted source uncertainties as described in the text. These blue histograms are much closer in value to the distribution of $V \mathrm{~s}$ such that $\sigma=1$. In our analysis, we maximized the number of grids for better differentiation when possible, but here only present the minimum $3 \times 3$ grid, where each grid contains 1620 sources.

(A color version of this figure is available in the online journal.)

Additional uncertainty exists in the PACS source extractions that is not accounted for in the variability parameter analysis above which results from the $1 / f$ noise in masked regions. The $1 / f$ noise in single epoch images causes striping in one direction, while combined-epoch images have striping in two perpendicular directions, causing a cross-hatching pattern. The noise is higher in the cross-hatched regions of the combined image with stripe intersections coadding to increase the noise. We isolated the hatching in order to measure the magnitude of this added uncertainty by subtracting the Starfinder-produced sky image from the residual image, thus removing the ISM structure from the residual image and leaving only the cross- hatching pattern and other instrumental noise. Point sources and ISM emission have been removed, so the flux within a point source-sized aperture should be zero. We calculated the flux within 10,000 apertures (aperture radius $=R_{80 \%}$, background annulus inner radius $=1.2 \times R_{80 \%}$, annulus width $=2$ pixels, where $R_{80 \%}$ is the radius which contains $80 \%$ of the psf's power) in both masked and unmasked regions of both galaxies' PACS images and measured the scatter about a flux of zero. The masked regions, which suffer strongly from striping, have an enhanced scatter (Figures 22 and 23), indicating a larger flux uncertainty for sources located in masked regions relative to those in unmasked regions. To account for this additional 

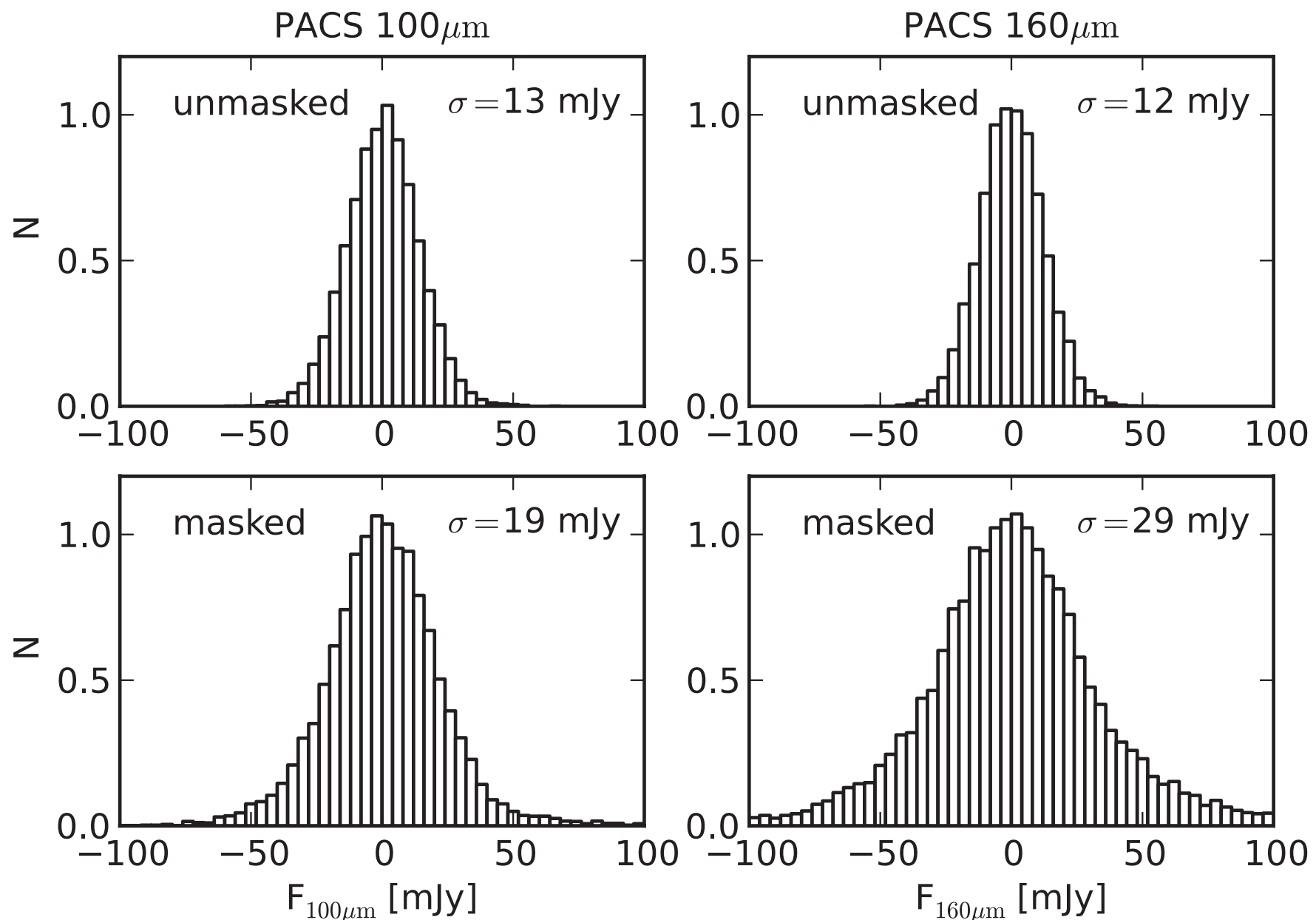

Figure 22. Histograms showing the distribution of the aperture photometry of 10,000 apertures on the LMC PACS residual minus sky image. The histograms are normalized to peak at $N=1$. PACS $100 \mu \mathrm{m}$ is shown to the left and PACS $160 \mu \mathrm{m}$ to the right. The scatter of the flux (quantified with the standard deviation, $\sigma$ ) is indicated in each panel for apertures in unmasked (top row) and masked (bottom row) regions.

Table 5

Uncertainty Correction Factors for the Combined-epoch Images

\begin{tabular}{|c|c|c|c|}
\hline$\lambda(\mu \mathrm{m})$ & $u_{\text {cor }}$ & $f_{\text {cor }}$ & $s_{\text {cor }}$ \\
\hline \multicolumn{4}{|c|}{ LMC } \\
\hline 100 & 3.00 & 0.065 & 0.25 \\
\hline 160 & 4.00 & 0.060 & 0.25 \\
\hline 250 & 1.40 & 0.060 & 0.35 \\
\hline 350 & 1.60 & 0.050 & 1.20 \\
\hline 500 & 1.50 & 0.070 & 2.50 \\
\hline \multicolumn{4}{|c|}{ SMC } \\
\hline 100 & 3.00 & 0.050 & 0.40 \\
\hline 160 & 3.00 & 0.065 & 0.55 \\
\hline 250 & 1.25 & 0.030 & 0.50 \\
\hline 350 & 1.20 & 0.050 & 1.20 \\
\hline 500 & 1.45 & 0.070 & 2.40 \\
\hline
\end{tabular}

uncertainty from the $1 / f$ noise, we have linearly added an uncertainty of ( $\left.\sigma_{\text {masked }}-\sigma_{\text {unmasked }}\right)$ to the uncertainty determined from the variability parameter, where $\sigma_{\text {masked }}$ is the standard deviation of the scatter about a flux of zero in masked regions, and $\sigma_{\text {unmasked }}$ is the same in unmasked regions. In the LMC, we have increased the PACS $100 \mu \mathrm{m}$ and $160 \mu \mathrm{m}$ uncertainties by $6 \mathrm{mJy}$ and $17 \mathrm{mJy}$, respectively. For the SMC, the same uncertainties are increased by $6 \mathrm{mJy}$ and $11 \mathrm{mJy}$. Recall that the difference in sensitivity between masked and unmasked regions is greater for PACS $160 \mu \mathrm{m}$ than for PACS $100 \mu \mathrm{m}$ (Table 3), consistent with the larger value of ( $\left.\sigma_{\text {masked }}-\sigma_{\text {unmasked }}\right)$ for PACS $160 \mu \mathrm{m}$ than PACS $100 \mu \mathrm{m}$.

The scatter about zero flux from this same "source-less" aperture analysis in SPIRE and unstriped PACS regions is a measure of the minimum uncertainty that results from the instrumental noise of the image. This is essentially the minimum value of $s$ (and therefore $\sigma$ ) from the variability parameter, as $\sigma=s$ when $F$ and $s k y$ are both 0 . The minimum uncertainty derived from the aperture analysis and that which results from the multiplication of $u n c$ and $u_{\text {cor }}$ show good agreement (within 1-3 mJy). Perfect agreement is not expected, as one is determined from an aperture photometry analysis while the other from Starfinder, but their close correspondence confirms reasonable values of $u_{\text {cor }}$.

In Figure 24 we plot the uncertainty of the fluxes as a function of flux for sources extracted from the PACS $100 \mu \mathrm{m}$ and SPIRE $250 \mu \mathrm{m}$ combined-epoch images. Lines of constant $\mathrm{S} / \mathrm{N}$ are drawn to guide the eye. Note that an upper limit of the $\mathrm{S} / \mathrm{N}$ is set by $f_{\text {cor }}$ at higher fluxes and $u_{\text {cor }}$ at low fluxes.

These uncertainty values provide an accurate assessment of the uncertainties of the fluxes in the HERITAGE catalogs. For detailed examination of particular regions, manual photometry on individual sources may improve the flux estimates, by allowing the user to vary the PSF, apply non-circular aperture 


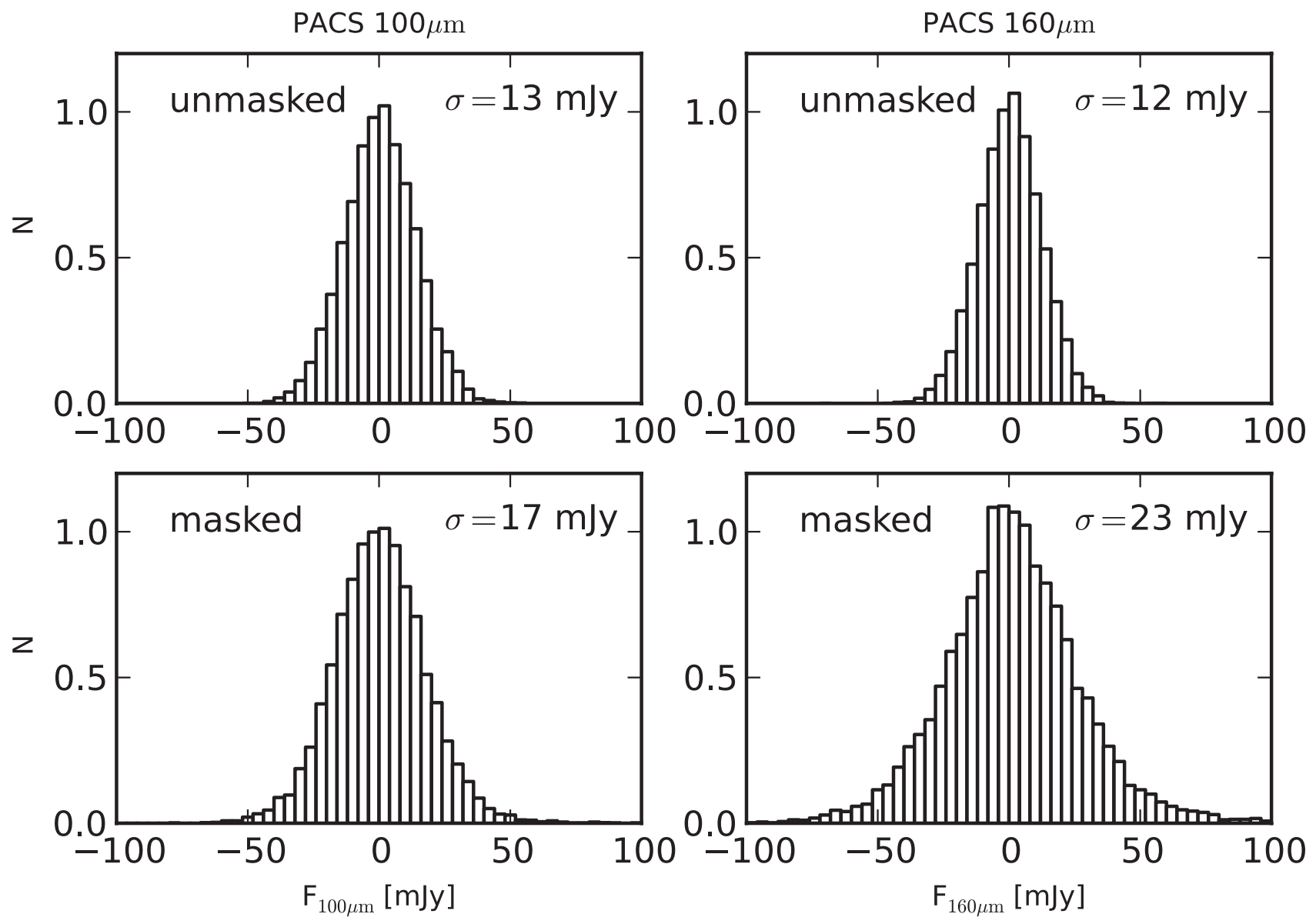

Figure 23. Same as Figure 22, but for the SMC.

photometry, or better determine the local background for each source. In the case of PACS, when possible, locally destriping the image before attempting photometry may improve both the flux and uncertainty.

\subsection{Simulated Source Tests}

A battery of simulated data tests was run on both the PACS and the SPIRE LMC data sets to assess completeness and photometric fidelity. A $5 \mathrm{deg}^{2}$ area centered at $85^{\circ} 7-69.8$ (R.A., decl.) was selected based on its wide range of background variability. The region was populated with a selection of 800 simulated sources. Both the combined-epochs image as well as the single epoch images were tested. The 800 sources consisted of eight flux bins each with 100 sources. The flux bins were 20, 50, 100, 200, 500, 1000, 2000 and $5000 \mathrm{mJy}$. Source locations were chosen to avoid locations within $2 \times \mathrm{FWHM}$ where known sources were extracted. We instituted this source location restriction so that we could assess the completeness as a function of background and not confusion from crowding. In these datasets, confusion with other sources is low compared to confusion with the high, complex background. For SPIRE, sources were inserted into the timeline datasets and subsequently those timeline data were re-mosaicked into map images from which the photometry was done. This was done so that we could simultaneously determine the value of the pixelization correction (SPIRE Photometry Cookbook $^{41}$ ). Pixelization correction values of $0.951,0.931$ and 0.950 were determined for SPIRE 250, 350, and $500 \mu \mathrm{m}$

\footnotetext{
41 http://herschel.esac.esa.int/twiki/pub/Public/SpireCalibrationWeb/
} SPIREPhotometryCookbook_apr2011_3.pdf data respectively. With the pixelization correction applied to the SPIRE results the photometry of simulated sources reproduced true fluxes well. No pixelization correction is required for PACS data, so sources were inserted directly onto the map images.

The simulated source tests found a tight agreement between true and extracted source fluxes. The ability of the photometric process to find and extract all simulated sources (the measurement of completeness) is a strong function of the background flux and complexity. For example, the left-hand panel of Figure 25 shows the completeness curve for sources on a low background, while the right-hand panel is the completeness curve for regions we define as high background (see Table 6 for the definition of high- and low-backgrounds). The overall completeness at a $90 \%$ level, as determined by these simulated data tests, is tabulated in Table 6. The completeness is clearly a function of background, and given the highly variable nature of the background, the completeness has substantial variability even within the high-background regions. These curves therefore represent the average completeness within the region, with the true completeness of a particular region depending on the brightness and complexity of the background immediately surrounding the source. Global LMC and SMC average 90\% completeness limits are calculated for each wavelength taking into account the filling-fraction of the galaxy with high and low surface brightness fluxes (Table 6). These average values underestimate the catalogs' completeness levels because while most of the image is low background, most sources are located in high background. Nevertheless, they provide rough guidelines for the limitations of the catalogs. 

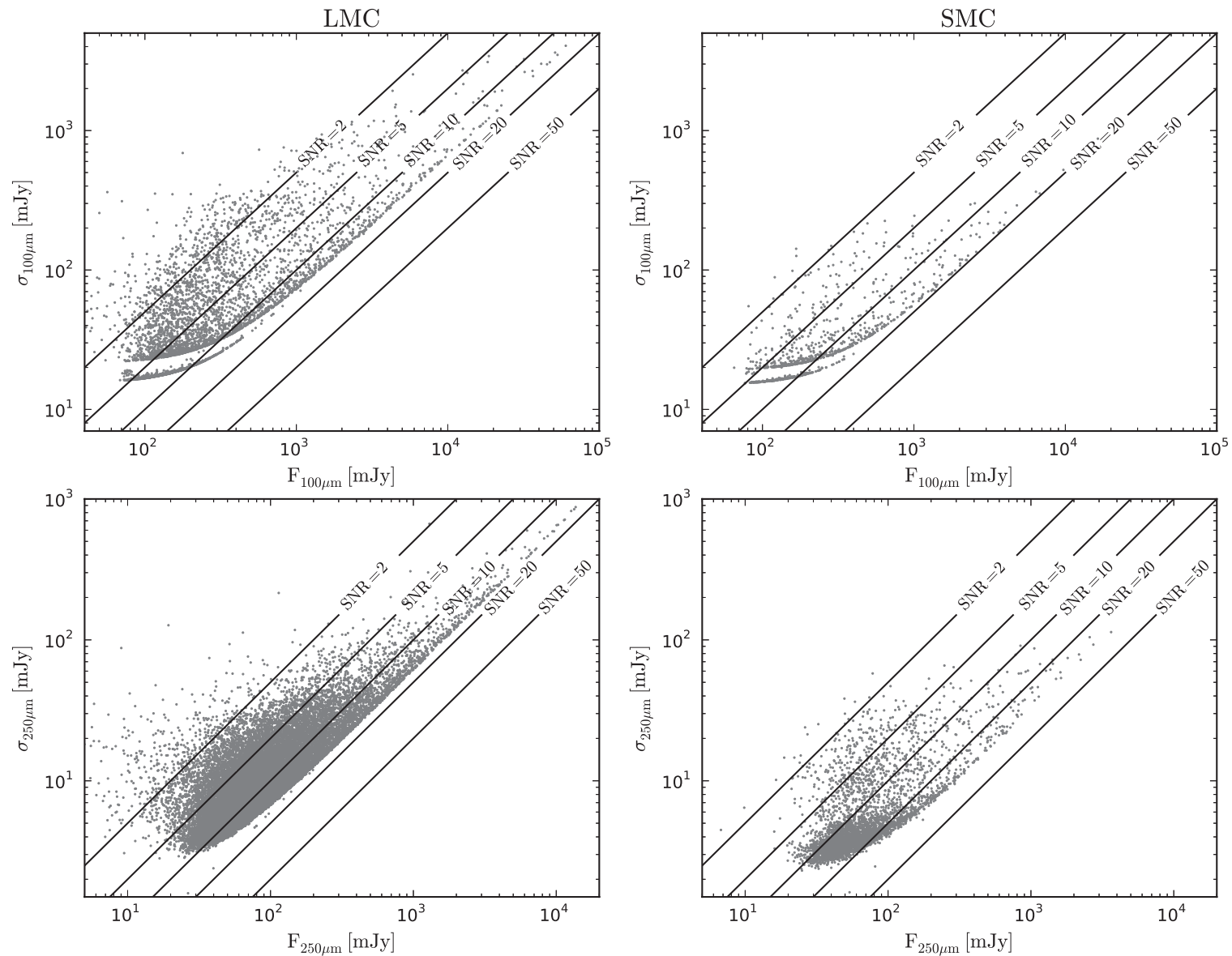

Figure 24. PACS $100 \mu \mathrm{m}$ (top row) and SPIRE $250 \mu \mathrm{m}$ (bottom row) catalog source flux uncertainty as a function of flux for the LMC (left), and SMC (right). Lines of constant $\mathrm{S} / \mathrm{N}$ are drawn to guide the eye.

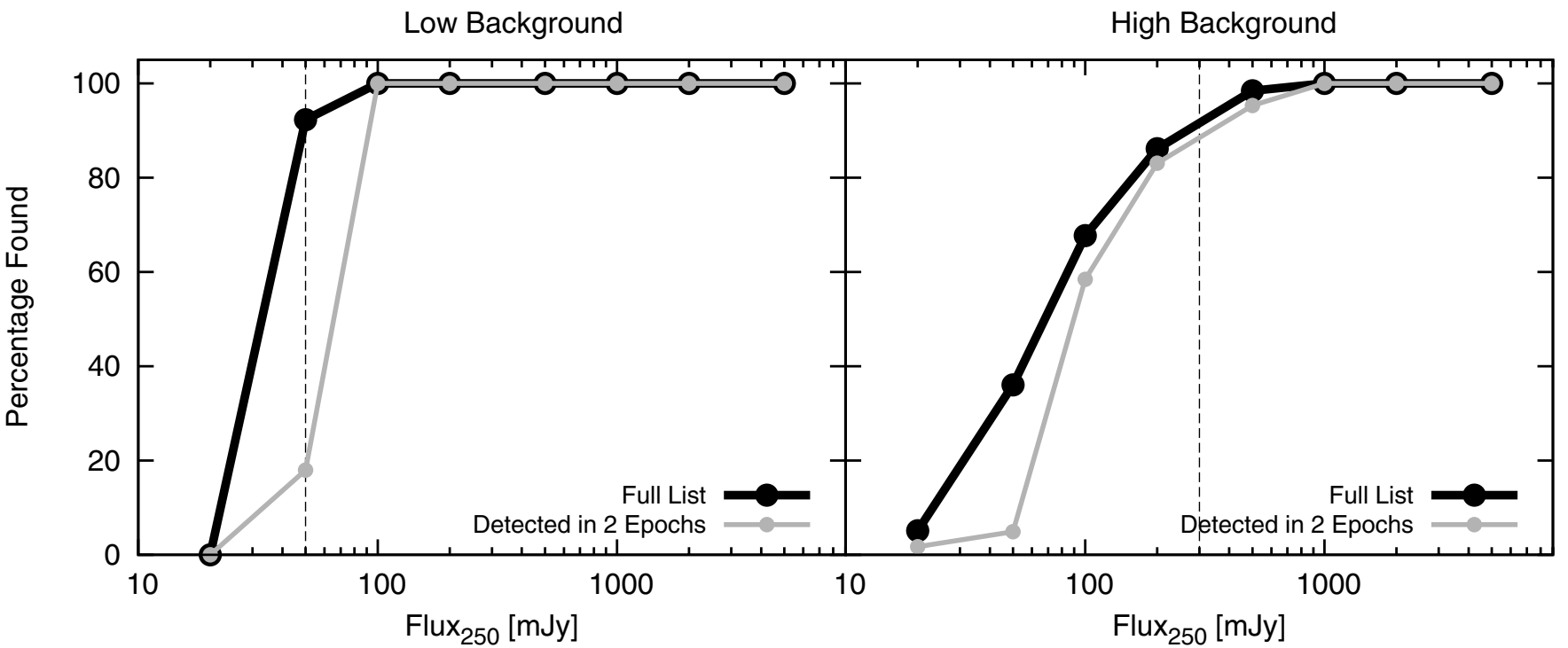

Figure 25. Completeness curves for SPIRE $250 \mu \mathrm{m}$ created from the false source injection test, which show the percentage of false sources recovered as a function of flux. The left and right panels are for sources within a low $\left(\leqslant 10 \mathrm{MJy} \mathrm{sr}^{-1}\right)$ and high $\left(>10 \mathrm{MJy} \mathrm{sr}^{-1}\right)$ background, respectively. The black curve is for all sources recovered from the combined image (i.e., the Full List), while the gray curve is Full List sources detected in both individual epoch images. The vertical lines mark the Full List $90 \%$ completeness limit at each background level.

\subsection{Source Extraction Assessment and Applicability}

To verify that Starfinder was producing reasonable results, we performed rough extractions using alternate source extraction packages including SExtractor (Bertin \& Arnouts 1996), CuTEx
(Molinari et al. 2011), and IRAF's Apphot (Tody 1993). Each of these packages extracts the flux and determines the background in a slightly different manner; Starfinder uses PSF fitting, CuTEx uses a 2D Gaussian fitting routine, and SExtractor and Apphot are aperture photometry routines. We ran each 
Table 6

Parameters of Extracted Point-source Catalogs

\begin{tabular}{|c|c|c|c|c|c|}
\hline \multirow[t]{2}{*}{ Parameter } & \multicolumn{2}{|c|}{$\operatorname{PACS} \lambda(\mu \mathrm{m})$} & \multicolumn{3}{|c|}{$\operatorname{SPIRE} \lambda(\mu \mathrm{m})$} \\
\hline & 100 & 160 & 250 & 350 & 500 \\
\hline \multicolumn{6}{|l|}{ LMC } \\
\hline Total number in extracted List & 8686 & 27136 & 48082 & 38482 & 12224 \\
\hline Number in catalog & 4164 & 9324 & 25445 & 22082 & 7355 \\
\hline FWHM of extracted source $\left({ }^{\prime \prime}\right)$ & 8.6 & 12.6 & 18.3 & 26.7 & 40.5 \\
\hline \multicolumn{6}{|l|}{ SMC } \\
\hline Total number in extracted list & 1353 & 5810 & 11923 & 11669 & 2335 \\
\hline Number in catalog & 898 & 1590 & 5465 & 5313 & 1069 \\
\hline FWHM of extracted source $\left({ }^{\prime \prime}\right)$ & 8.8 & 12.6 & 18.3 & 26.7 & 39.7 \\
\hline \multicolumn{6}{|l|}{$90 \%$ completeness limits from simulated source tests } \\
\hline Low background $\left(\mathrm{MJy} \mathrm{sr}^{-1}\right)$ & $\leqslant 15$ & $\leqslant 25$ & $\leqslant 10$ & $\leqslant 5$ & $\leqslant 2.5$ \\
\hline Low background $90 \%$ completeness limit (mJy) & 450 & 150 & 50 & 50 & 90 \\
\hline High background (MJy sr $\left.{ }^{-1}\right)$ & $>15$ & $>25$ & $>10$ & $>5$ & $>2.5$ \\
\hline High background $90 \%$ completeness limit (mJy) & 450 & 400 & 300 & 400 & 400 \\
\hline LMC average $^{\text {a }} 90 \%$ completeness limit (mJy) & 450 & 190 & 90 & 110 & 140 \\
\hline SMC average ${ }^{a} 90 \%$ completeness limit (mJy) & 450 & 160 & 60 & 60 & 100 \\
\hline
\end{tabular}

Note. ${ }^{\text {a }}$ The average is computed as $C_{\text {total }}=C_{\text {low }} * f_{\text {low }}+C_{\text {high }} * f_{\text {high }}$, where $C$ is the $90 \%$ completeness limit, $f$ is the filling factor across the galaxy, and the subscripts low and high reference the corresponding background level.

package on a subregion of the LMC that contained both highand low-background regions to gauge the consistency between the methods, and found each package to return source fluxes that generally agreed to within a factor of $\sim 1.5$, with the largest discrepancies for sources in the brightest, most complex backgrounds. Sources in more isolated regions of the SPIRE $250 \mu \mathrm{m}$ map generally agree to within $20 \%$. The tests confirm that Starfinder returns reasonable results, and affirms that one of the primary sources of flux uncertainty is the variable background.

As described in Section 7.2, simulated source tests revealed a tight agreement between the Starfinder-extracted and true fluxes. This is perhaps unsurprising as the inserted sources' PSF matched that used during extraction by Starfinder. For real sources, this is not necessarily the case; indeed, we find some variation in PSF width for the HERITAGE sources (see above). The Starfinder post-extraction residual images (i.e., the original image minus the PSF fits) show some sources with unextracted, residual flux at the position of a source. These sources have extended emission beyond the PSF such that while the bright point-like center of the source is removed, the lower level, more extended flux is not. The inclusion of this residual flux in the source's flux depends on the interpretation and science goals of the user. Many of the sources identified in the HERITAGE images are embedded in large-scale dusty structures, and the sources themselves may not be discrete compact structures, but rather localized bright, dusty density enhancements. Such a source may not be strictly point-like, and aperture photometry may be better suited for such sources, but decisions about using PSF-fitted or aperture photometry must be made on a source-by-source basis. Moreover, the varying PSF width may require a variable aperture correction factor. No single photometric method will fill all desired photometric roles, however we found the Starfinder results produce good results for true point sources and lower bounds for sources within areas of high complexities. To designate good point sources we fit a 2D Gaussian at each source location. To isolate the source and minimize any background effects on the 2D Gaussian fit we used the residual image (sources removed except the desired source) with the background image removed. We tabulate the
Table 7

Flux Flag Statistics: Units are Percentage of Sources in the Catalogs with Each Flux Flag Value

\begin{tabular}{lcccc}
\hline \hline$\lambda(\mu \mathrm{m})$ & Flag $=1$ & Flag $=2$ & Flag $=3$ & Flag $=4$ \\
\hline \multicolumn{5}{c}{ LMC } \\
\hline 100 & 44 & 9 & 7 & 39 \\
160 & 31 & 4 & 12 & 54 \\
250 & 35 & 16 & 34 & 13 \\
350 & 29 & 20 & 26 & 24 \\
500 & 21 & 23 & 39 & 16 \\
\hline & \multicolumn{5}{c}{ SMC } & & \\
\hline 100 & 73 & 5 & 7 & 15 \\
160 & 66 & 2 & 7 & 24 \\
250 & 74 & 4 & 17 & 4 \\
350 & 65 & 10 & 20 & 5 \\
500 & 54 & 12 & 26 & 8 \\
\hline
\end{tabular}

results as a FWHM value in our catalog (averaging the $x$ and $y$ FWHM values), sources where the Gaussian fit failed (in either direction, or both) are designated with the value of 0 .

These FWHM values determine a photometric quality flag, "flux flag," in our catalog indicating the degree to which the source is point-like and thus the reliability and applicability of the flux measurement returned by Starfinder. The flag has a value of $1,2,3$, or 4, and Table 7 documents how many sources in each galaxy and waveband are assigned each flag. A flux flag of 1 is given to sources that have a measured FWHM consistent with the FWHM of the PSF $\left(\mathrm{FWHM}_{\text {source }}<1.1 \times \mathrm{FWHM}_{\mathrm{PSF}}\right)$. The flux for flux flag $=1$ sources is therefore a robust accounting of the sources' brightnesses.

A flux flag of 2 is assigned to sources for which the FWHM determination failed, but the source has a $\mathrm{S} / \mathrm{N}$ of greater than 5 . The great majority of flux flag $=2$ sources are faint, causing the FWHM measurement to fail from a poor Gaussian fit. Bright flux flag $=2$ sources tend to fail FWHM measurement due to their location within areas of complex background. For SPIRE, this constitutes a small fraction of sources; $0.6 \%$ of 250,350 , and $500 \mu \mathrm{m}$ sources are brighter than $0.5 \mathrm{Jy}$ and have a flux flag of 2. Because the PACS sensitivity is poorer, fewer faint 
sources have a $\mathrm{S} / \mathrm{N}>5$ (one of the flux flag=2 requirements), and thus there is a higher fraction of bright flux flag $=2$ sources; $\sim 4 \%$ of PACS sources are brighter than $0.5 \mathrm{Jy}$ and have a flux flag of 2.

In Figure 26, we present small image cutouts of the area around typical sources identified in the SPIRE $250 \mu \mathrm{m}$ image. The sources are organized by flux flag and flux. Note that faint flux flag $=2$ sources are located in relative isolation and that the residual images show little to no signs of under or over extraction. Conversely, bright sources are located in regions of more complex background and often show signs of residual flux surrounding the source. In these cases, Starfinder has extracted the central point-like core of the source, leaving the more extended surrounding envelope, and whether the extended emission should be included in the source flux depends on the science case. We leave it to the user to determine if the extended emission should be included in the source's photometry, in which case the user may wish to increase the uncertainty of the Starfinder extracted flux or treat it as a lower limit. Again, this constitutes a very small fraction of the Catalog, so most flux flag $=2$ source fluxes can be treated as good measurements.

Flux flag of 3 denotes sources that have a measured FWHM greater than that of the PSF $\left(\mathrm{FWHM}_{\text {source }}>1.1 \times \mathrm{FWHM}_{\mathrm{PSF}}\right)$ and a $\mathrm{S} / \mathrm{N}>5$. These sources are extended beyond the PSF, and thus Starfinder has extracted the point-like central core of the source (see Figure 26). In some cases the user may wish to include this extended flux, in which case aperture photometry may be more useful and the Starfinder flux can be considered a lower limit. Sources with a $\mathrm{S} / \mathrm{N}<5$ are marked with a flux flag of 4 , and have uncertainties that are dominated by the brightness and complexity of the background and for some PACS sources $1 / f$ noise; thus the extracted flux is highly uncertain. Note in Figure 26 the highly complex nature of these sources' environments. Because the sources in the catalogs we provide the community have passed fairly rigorous tests to verify the sources are real (see below), flux flag $=4$ sources should be considered true sources with highly uncertain or unknown fluxes. The fluxes provided in the catalogs for flux flag $=4$ should be treated with caution, and a user may wish to do a more detailed source-by-source extraction to obtain a higher fidelity measurement.

Note from Table 7, that there is a larger percentage of flux flag $=4$ PACS sources than SPIRE sources. This is a result of the poorer PACS sensitivity, particularly in the masked, striped regions where a large fraction of the sources reside. There is also a higher percentage of flux flag $=4$ sources in the LMC than the SMC due to the LMC's higher sky levels. Finally, note that the number of flux flag $=1$ sources generally decreases with increasing wavelength while the number of flux flag $=2$ or 3 sources increases. As the observed wavelength increases, the emission surrounding point sources originates from cooler, more extended dust, thus broadening the FWHM and relegating more sources to flux flag $=2$ or 3 status.

\subsection{Assessing the Reliability of Sources}

Table 6 documents the number of sources identified in each combined-epoch image by Starfinder. These sources represent the "Full List" of point sources, every source identified in the combined-epoch images. We provide for use by the community a subsample of the Full List that has been vetted for reliability, which we call the "Catalog." The Catalogs contain only combined-epoch sources that pass the reliability tests detailed below. The Full List may contain sources-particularly at low flux levels - that are false detections. Moreover, because of the bright, highly variable, and complex background present in our images, it is necessary to assess the point-like nature of sources to separate true point sources from marginally point-like fluctuations in the background. The most reliable point sources are those whose point-like emission is quite distinct from the background. Table 6 documents the number of vetted Catalog sources identified in each combined-epoch image. The HERITAGE Catalogs are available electronically from the Herschel Science Center, and in the Appendix we document the format and column description of the catalogs.

Our assessment of the reliability of the point sources is a two-step process. First we position matched the Full List for a particular waveband to the sources identified in the single epoch images of the same waveband. We call this procedure "cross-epoch matching." A matching radius of $0.7 \times$ FWHM is adopted. A source is more reliable if it is identified in both the combined image and one or more of the individual epochs. False or low $\mathrm{S} / \mathrm{N}$ sources are less likely to be identified in the individual epochs, which are noisier than the combined image, and marginally point-like sources are less likely to be identified as point sources in both the combined epoch and the individual epochs. Thus, matching to the individual epoch lists tends to identify the brightest point-like sources. Figure 25 displays results of the aforementioned simulated source extraction on the SPIRE $250 \mu \mathrm{m}$ image and compares the percentage of all recovered sources detected in the combined image (black line) with those detected in the combined image and both individual epochs (gray line). The black line shows the percentage of real sources recovered during source extraction, and thus represents the completeness of the Full List as a function of source flux. Note that bright sources are identified in the combined image and both individual epochs, while the dimmest sources are only detected in the combined image and one or no individual epochs.

The second step in the source reliability procedure, a source position match across different mid-infrared (mid-IR) and FIR wavelengths, is an effort to identify real sources not detected in multiple epochs. We call this procedure "cross-band matching." We have position matched the combined-image Full List from each waveband to sources in the other Herschel combined-image Full Lists and the mid-IR Spitzer SAGE catalogs at 24, 70, and $160 \mu \mathrm{m}$. Because of the large variation in angular resolution between the wavebands, we only match images with angular resolutions that agree within a factor of $\sim 1.5$. For each matching pair, a matching radius of $0.7 \times \mathrm{FWHM}_{\max }$ is adopted, where FWHM $_{\text {max }}$ is the larger FWHM of the two images. Table 8 documents the source lists used in the cross-band matching for each Herschel Full List.

After completion of both of these matching procedures (cross-epoch and cross-band matching), we identify the most reliable sources to be included in the "Catalogs" as follows. The reliability is determined by adding $n_{\text {epoch }}$, the number of individual epochs a Full List source is identified in $\left(n_{\text {epoch }}=0\right.$, 1,2 , or 3 ), and $n_{\text {band }}$ the number of images for which there is a cross-band match $\left(n_{\text {band }}=0,1,2\right.$, or 3$)$. If the sum is 2 or greater, the source is reliable and categorized as a "Catalog" source. All other sources are less reliable and are included in the Full List, which includes every source identified in the combined-epoch images. The Full List is not delivered to the Herschel Science Center because it is not as reliable as the Catalog; however, it can be accessed by contacting the HERITAGE team. The Catalog of sources at a particular wavelength therefore contains only combined-epoch sources that (1) are detected in at least two 


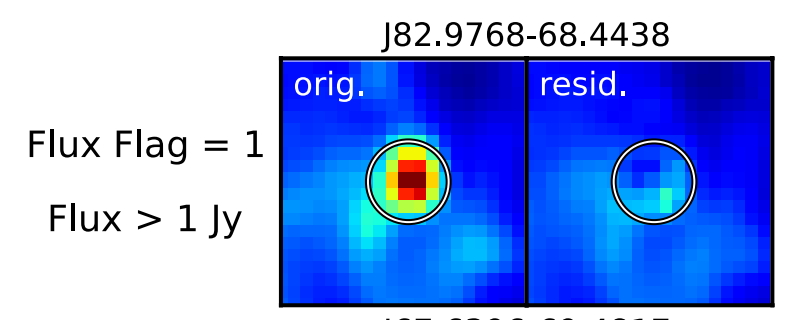

J67.6396-69.4817

Flux Flag $=1$

Flux $<0.5 \mathrm{Jy}$
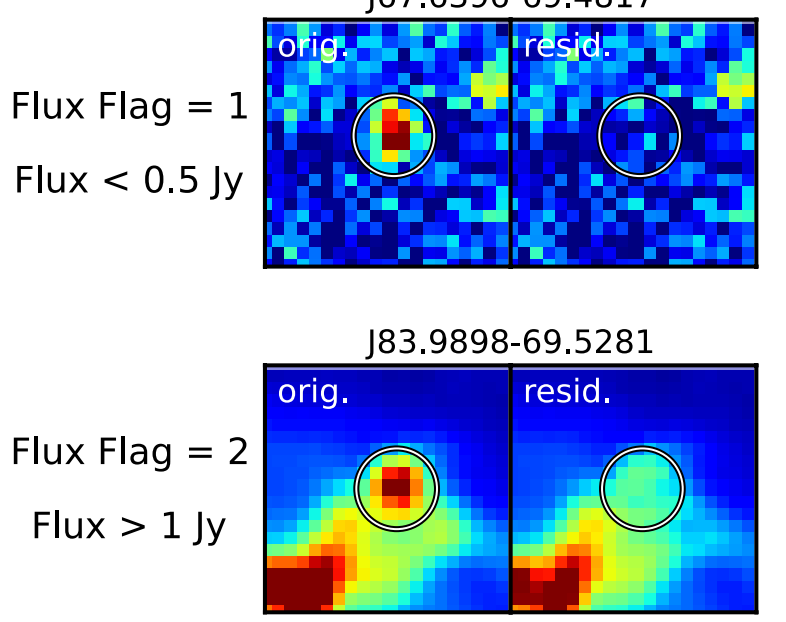

J68.3719-71.5101
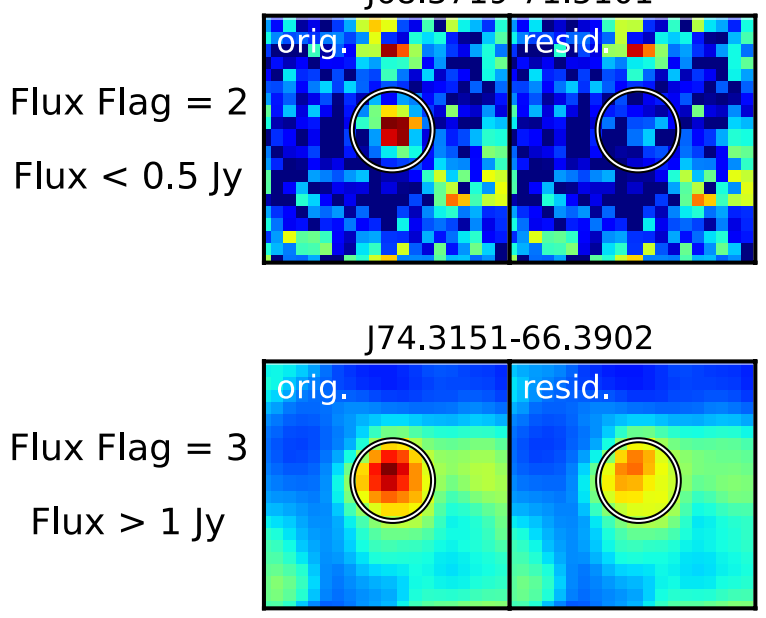

J69.1126-69.6793
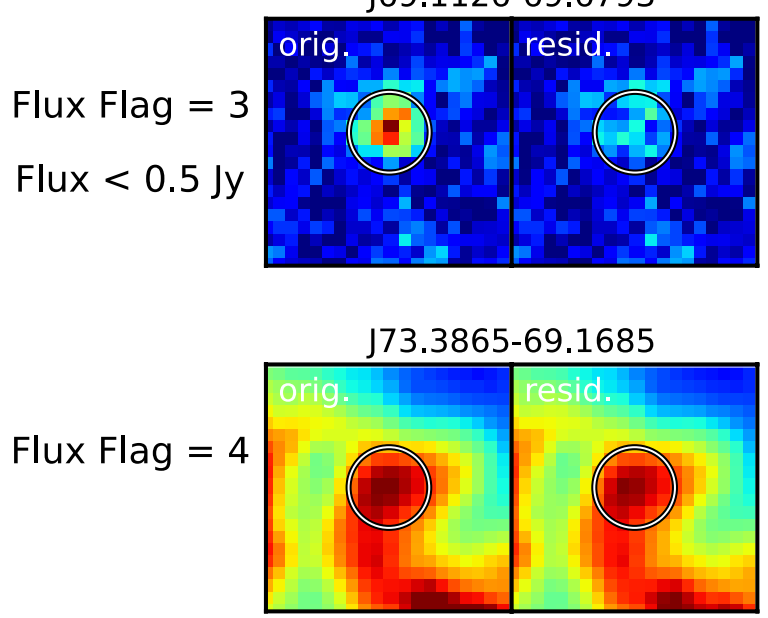

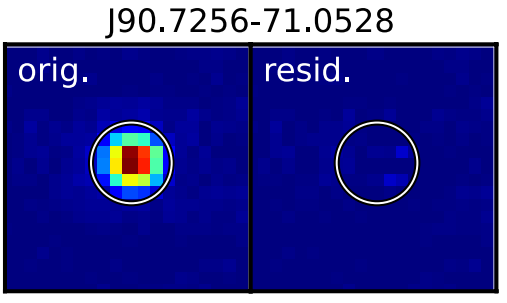

J66.7948-70.1219
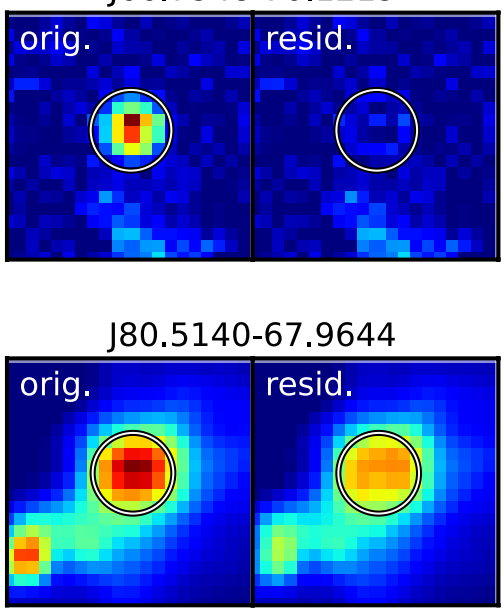

J72.2033-68.4036
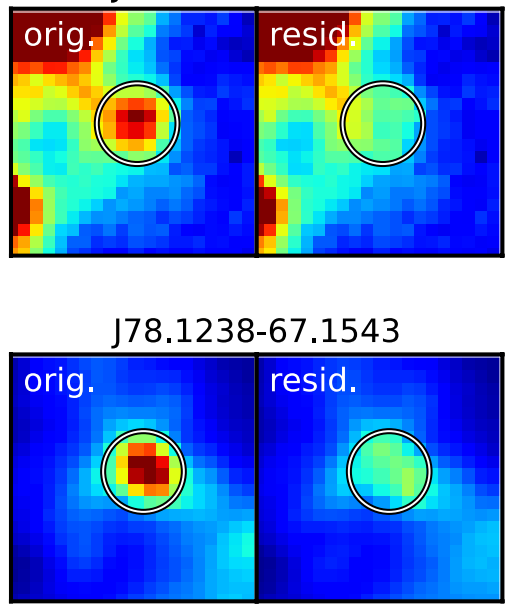

J70.3108-65.7365

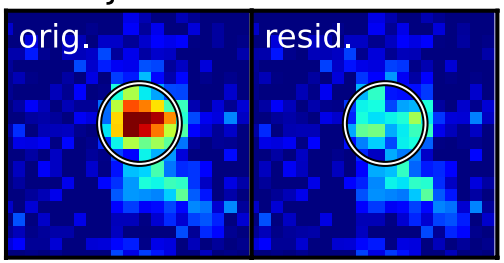

J74.3694-66.2470

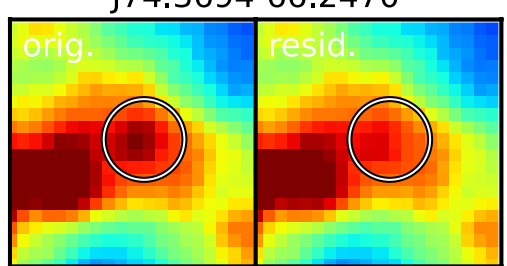

Figure 26. Image cutouts showing the combined-epoch SPIRE $250 \mu \mathrm{m}$ image around a source before (original image; left) and after (residual image; right) source extraction. The name of the source is shown above each image pair and the position of the source is marked with a circle. Sources are organized by flux flag and flux as shown to the left. 


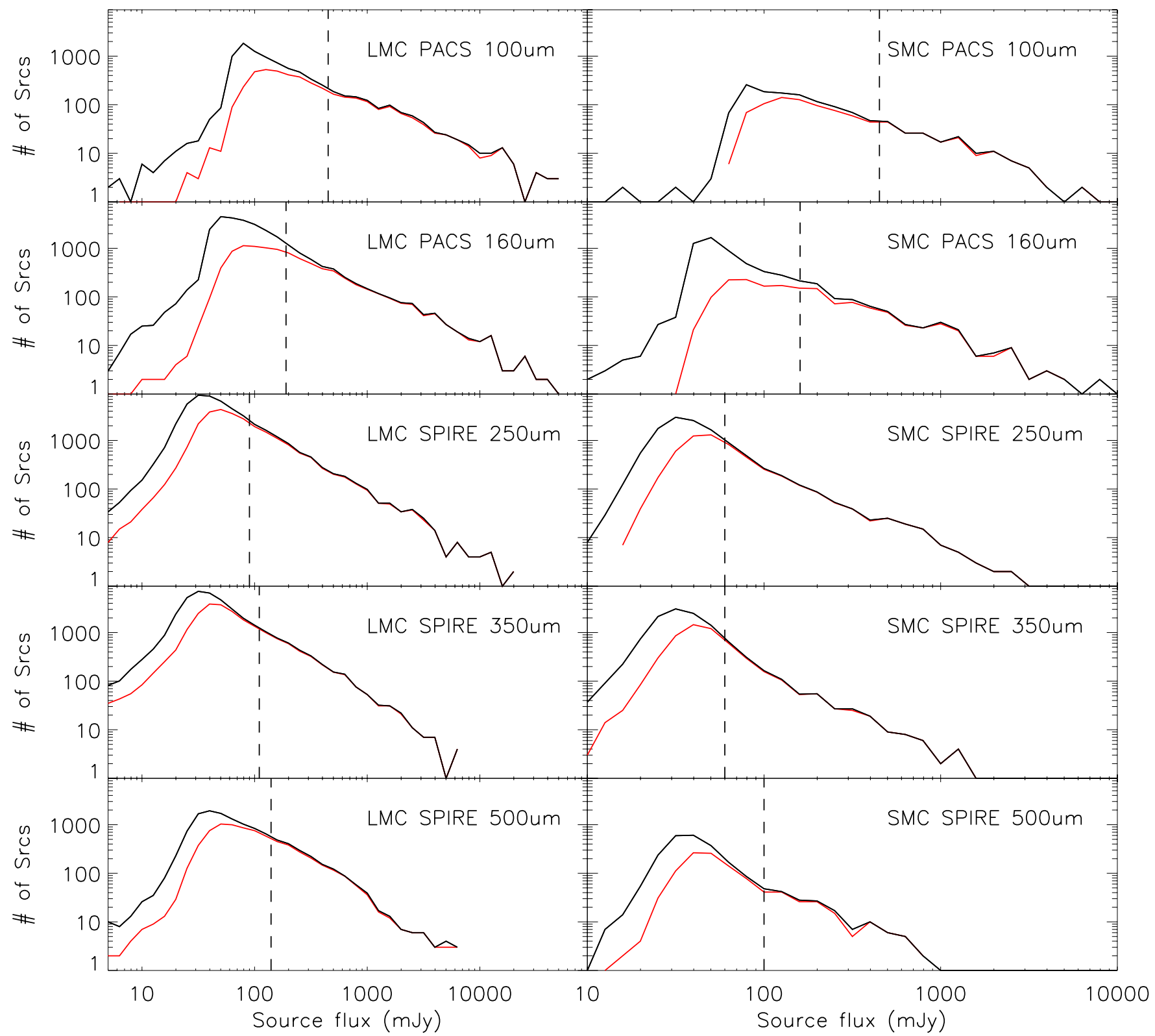

Figure 27. Plotted are the distribution of extracted sources as a function of flux for the LMC (left) and SMC (right). The red curves are only sources classified as Catalog sources, and the black curves are for all extracted sources (Full List sources). Data were binned logarithmically with bin size of 0.1 . The average $90 \%$ completeness level of the catalog (Table 6) is marked by the vertical dashed line. This average $90 \%$ completeness level is computed as a weighted average of the highand low-background values and underestimates the real 90\% completeness level (see text in Sections 7.2 and 7.4).

(A color version of this figure is available in the online journal.)

Table 8

Cross-band Matching

\begin{tabular}{ll}
\hline \hline Full List Waveband & \multicolumn{1}{c}{ Lists Matched To } \\
\hline PACS 100 & PACS 160 Full List, MIPS 24 Catalog \\
PACS 160 & PACS 100 Full List, SPIRE 250 Full List, MIPS 70 Catalog \\
SPIRE 250 & PACS 160 Full List, SPIRE 350 Full List, MIPS 70 Catalog \\
SPIRE 350 & SPIRE 250 Full List, SPIRE 500 Full List, MIPS 70 Catalog \\
SPIRE 500 & SPIRE 350 Full List, MIPS 160 Catalog
\end{tabular}

single epoch images of the same wavelength, (2) are identified in at least two other wavelengths in combined-epoch Herschel or Spitzer images, or (3) are detected in one single epoch image of the same wavelength and at least one other combined-epoch image at a different wavelength. For example, a PACS $100 \mu \mathrm{m}$ source that is detected in two PACS $100 \mu \mathrm{m}$ single epoch images $\left(n_{\text {epoch }}=2\right)$ and has a coincident source in the PACS $160 \mu \mathrm{m}$ combined-epoch image $\left(n_{\text {band }}=1\right)$ has $n_{\text {epoch }}+n_{\text {band }}=3$, and is a Catalog source. Conversely, a PACS $100 \mu \mathrm{m}$ source not detected in any individual epochs and without a coincident PACS $160 \mu \mathrm{m}$ or MIPS $24 \mu \mathrm{m}$ source is unreliable, a possible false detection, and is included only on the Full List. Table 6 

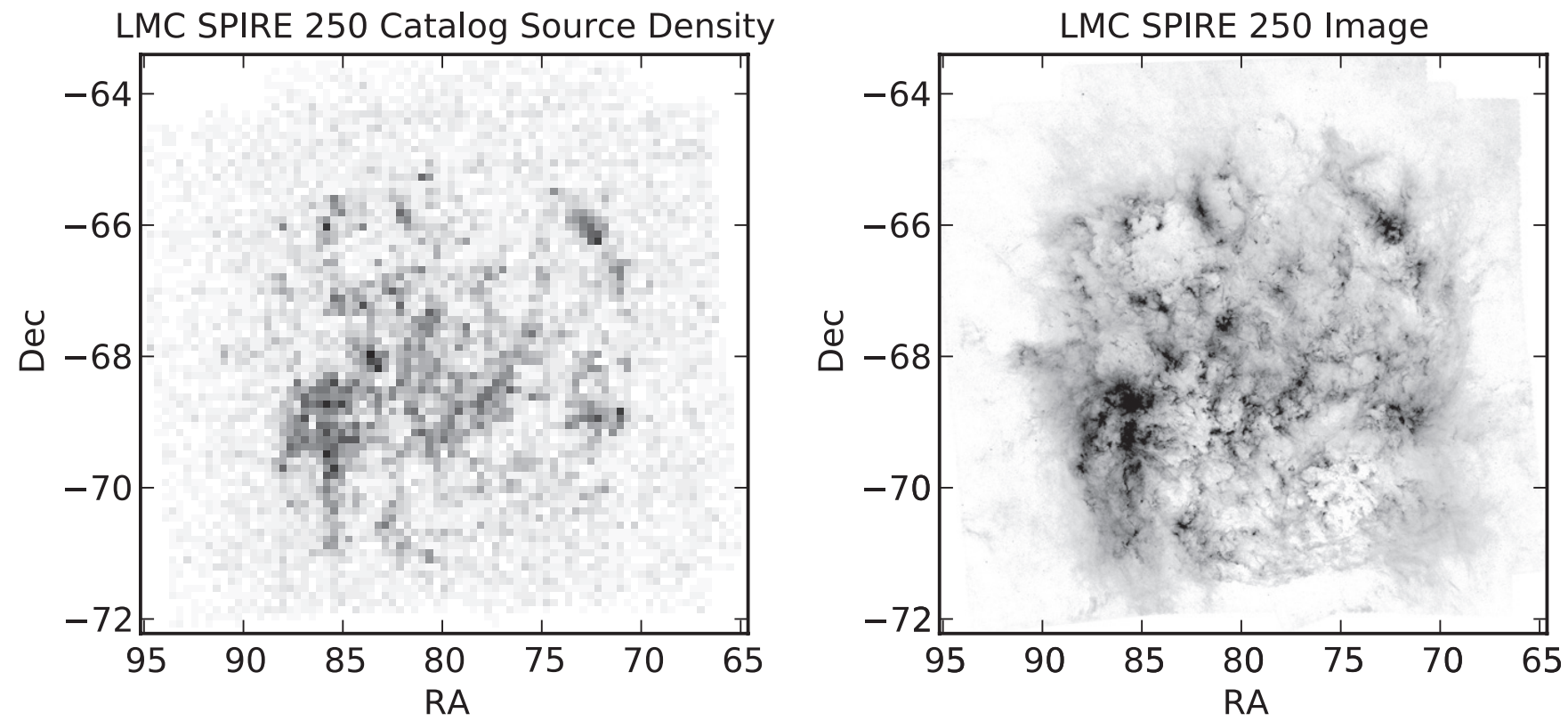

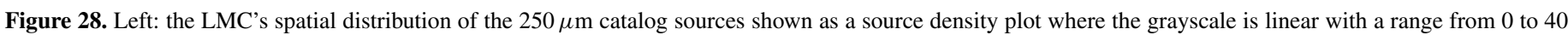

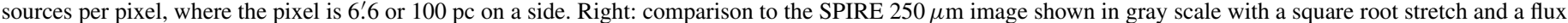
range of $0-100 \mathrm{MJy} \mathrm{sr}^{-1}$.
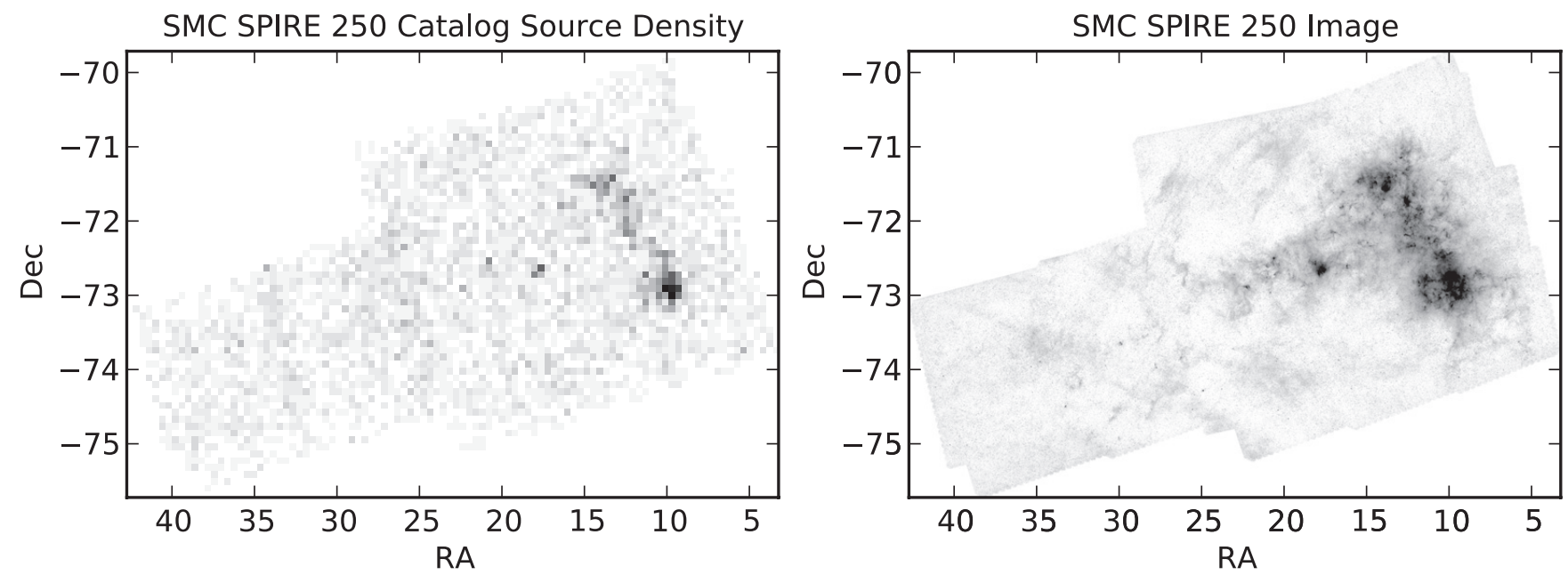

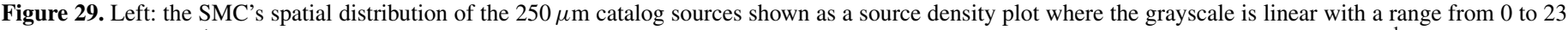
sources per pixel is 5.8 or $100 \mathrm{pc}$ on a side. Right: comparison to the SPIRE $250 \mu \mathrm{m}$ image shown in gray scale with a flux range of $0-36 \mathrm{MJy}$ sr ${ }^{-1}$.

documents the number of Catalog sources, that passed all this screening, for each combined-epoch image. The Catalog sources represent between $27 \%$ and $66 \%$ of the Full List sources across the five bands of HERITAGE.

In Figure 27 we present the histogram of the number of LMC and SMC sources as a function of flux (in mJy) as well as reliability classification (Catalog or Full List). As expected, the Catalog histogram (in red) cuts off at higher fluxes than the Full List histogram (in black). Using the results of the false source extraction procedure described above, we can estimate the completeness of the Catalog at low background. Since the black line in Figure 25 is the completeness curve for the Full List, and the gray curves show the completeness for sources detected in both epochs, the Catalog must lie somewhere between the two. The Catalog's creation relies on matching across wavebands, so the precise shape of the Catalog completeness curve for a given population depends on the SED of that population. For the SPIRE $250 \mu \mathrm{m}$ sources, the test suggests that the Catalog is complete above $100 \mathrm{mJy}$ at low background, with completeness quickly dropping below that level. Recall from above that the completeness is highly dependent on the background flux and complexity. The vertical dashed lines in Figure 27 represent the average $90 \%$ completeness limits from Table 6 and provide a rough guide to the limitation of the catalogs across each galaxy.

\section{DUSTY POPULATIONS IN THE LMC AND SMC}

The extracted sources in the PACS and SPIRE bands represent some of the dusty inhabitants of the LMC and SMC as well as background galaxies. The flux histograms of all the PACS and SPIRE bands show that the SPIRE $250 \mu \mathrm{m}$ band is the most sensitive and the PACS $100 \mu \mathrm{m}$ is the least sensitive. Thus using the SPIRE $250 \mu \mathrm{m}$ source counts as a guide, we find $\sim 25,000$ sources in the LMC and $\sim 5500$ in the SMC. These numbers are large in comparison to current massive YSO candidate lists ( $\sim 1800$ in the LMC and $\sim 1200$ in the SMC) based on Spitzer-only data (see Whitney et al. 2008; Sewiło et al. 2013). Figures 28 and 29 display the spatial distribution of the SPIRE 
$250 \mu \mathrm{m}$ catalog with respect to the ISM dust emission at SPIRE $250 \mu \mathrm{m}$. The majority of sources show a strong concentration in the bright filaments of dust-emitting ISM; however, there is also a background of some uniformly distributed sources. Analysis and characterization of these populations will be covered by J. Seale et al. (in preparation). However, our initial results from HERITAGE indicate that those sources associated with the LMC are most likely dominated by YSOs (e.g., Sewiło et al. 2010) and some may be associated with dust clumps (e.g., Kim et al. 2010). We expect that there are only a few massive and very dusty evolved stars (e.g., Boyer et al. 2010; Clayton et al. 2011), and one of these is the youngest supernova remnant, SN 1987A (Matsuura et al. 2011).

\section{HIGHER LEVEL HERITAGE DATA PRODUCTS FOR THE COMMUNITY}

We have delivered several higher level data products to the Herschel Science Center that are available to the astronomical community. Combined-epoch images of the LMC and SMC, which are shown in Figures 13 and 15, are available in all the PACS and SPIRE bands of HERITAGE: 100, 160, 250, 350 and $500 \mu \mathrm{m}$. The source extractions are delivered in the form of catalogs. Although cross-band and cross-epoch matching was used to assess the reliability of the source, the source extractions are made available as independent lists for each of the five HERITAGE bands. In the J. Seale et al. (in preparation) paper, a cross-band matched catalog will be discussed and published for use by the astronomical community. We anticipate that these publicly available data products will further the science done with HERITAGE data, beyond what the team had envisioned.

\section{SUMMARY}

In this paper, we presented an overview of the Herschel HERITAGE project, which surveyed the LMC and SMC in five bands including PACS 100 and $160 \mu \mathrm{m}$ and SPIRE 250, 350, and $500 \mu \mathrm{m}$ wavelengths. We described the observing strategy and data processing particularly for PACS, which resulted in maps of these galaxies in all five bands. The emission in these Herschel bands arises from the ISM dust emission from these galaxies. Its spatial distribution is more similar to the ionized atomic and molecular tracers than the stellar population. The total fluxes of the galaxies in these five Herschel bands are reported and agree very well with prior total flux measurements, except the SMC PACS $160 \mu \mathrm{m}$ band slightly exceeds the Spitzer/MIPS $\mu \mathrm{m}$ measurement. We have created source extraction catalogs for the LMC and SMC in all five bands. The SPIRE $250 \mu \mathrm{m}$ catalogs are the most sensitive and have $\sim 25,000$ sources in the LMC and $\sim 5500$ in the SMC. Our first papers, based largely on the LMC SDP strip (Meixner et al. 2010), demonstrate the potential this survey will have for studies in the LMC and SMC. These first papers included studies of supernova remnants (Matsuura et al. 2011; Otsuka et al. 2010), evolved stars (Boyer et al. 2010), YSOs (Sewiło et al. 2010; Clayton et al. 2010), H II regions (Hony et al. 2010), dust clumps (Kim et al. 2010), dust mass and submillimeter excess (Gordon et al. 2010; Galliano et al. 2011), and dust-to-gas mass ratios and related issues (RomanDuval et al. 2010). We anticipate future papers on these same topics across the whole of the LMC and SMC.

We acknowledge financial support from the NASA Herschel Science Center, JPL contract Nos. 1381522 and 1381650. M.R. is supported by FONDECYT No1080335 and FONDAP No15010003. F.K. acknowledges support from the National Science Council in the form of grant NSC100-2112-M-001-023MY3. R.A.S. acknowledges financial support from the NASA Herschel Science Center, JPL contract No. 1350371. We are thankful for the contributions and support from the European Space Agency (ESA), the PACS and SPIRE teams, the Herschel Science Center (esp. L. Conversi) and the NASA Herschel Science Center (esp. A. Barbar and R. Paladini), and the PACS and SPIRE Instrument Control Centers (esp. George Bendo), without which none of this work would be possible.

\section{APPENDIX}

\section{MAKEUP OF HERITAGE POINT-SOURCE CATALOGS}

Tables 9-13 document the columns provided in the HERITAGE point-source Catalogs and describe the columns' contents. The catalog for each waveband and galaxy is provided separately, and are available from the Herschel Science Center. Source name convention is an 11 character string, OOOIIPPPPEL, followed by source-specific position designation JRR.RRRRSDD.DDDD. OOO denotes the observatory,

Table 9

PACS $100 \mu \mathrm{m}$ Catalog Contents

\begin{tabular}{|c|c|c|c|}
\hline Column & Name & Description & Null \\
\hline 1 & Source name & The identifying name of the source. A description of source name construction is found above & $\ldots$ \\
\hline 2 & R.A. (J2000) & Right ascension, J2000 (deg) & $\ldots$ \\
\hline 4 & dRA & Right ascension uncertainty (arcsec) & $\cdots$ \\
\hline 5 & dDecl. & Declination uncertainty (arcsec) & $\ldots$ \\
\hline 6 & flux & Starfinder-extracted flux (mJy) & $\ldots$ \\
\hline 8 & flagFlux & Flag describing the quality of the flux measurement (see Section 7.3) & $\ldots$ \\
\hline 9 & flagStripe & Flag notes if source is in masked region and may suffer from striping. 1 for masked; 0 for unmasked & $\ldots$ \\
\hline 10 & uncSF & Starfinder-estimated uncertainty of flux (mJy) & $\ldots$ \\
\hline 11 & sky & Starfinder-determined sky brightness $\left(\mathrm{MJy} \mathrm{sr}^{-1}\right)$ & $\cdots$ \\
\hline 12 & fwhm & Full-width-at-half-maximum (arcsec) (see Section 7.3) & 0 \\
\hline 13 & flagConf & Confusion flag (see Section 7). 1 for confused; 0 for not confused & $\ldots$ \\
\hline 14 & corr & Starfinder correlation parameter (see Section 7) & $\ldots$ \\
\hline 16 & p160 & Number of PACS $160 \mu \mathrm{m}$ Full List sources within $8^{\prime \prime} .8$ & $\ldots$ \\
\hline 17 & $\mathrm{~m} 24$ & Number of MIPS $24 \mu \mathrm{m}$ Catalog sources within $6.0 / 6.2$ in the LMC/SMC & $\ldots$ \\
\hline
\end{tabular}


Table 10

PACS $160 \mu \mathrm{m}$ Catalog Contents

\begin{tabular}{|c|c|c|c|}
\hline Column & Name & Description & Null \\
\hline 1 & Source name & The identifying name of the source. A description of source name construction is found above & $\ldots$ \\
\hline 2 & R.A. (J2000) & Right ascension, J2000 (deg) & $\ldots$ \\
\hline 3 & Decl. (J2000) & Declination, J2000 (deg) & $\ldots$ \\
\hline 4 & dRA & Right ascension uncertainty (arcsec) & ... \\
\hline 5 & dDecl. & Declination uncertainty (arcsec) & .. \\
\hline 6 & flux & Starfinder-extracted flux (mJy) & .. \\
\hline 7 & unc & Uncertainty of flux (mJy) & $\cdots$ \\
\hline 8 & flagFlux & Flag describing the quality of the flux measurement (see Section 7.3) & $\cdots$ \\
\hline 9 & flagStripe & Flag notes if source is in masked region and may suffer from striping. 1 for masked; 0 for unmasked & .. \\
\hline 10 & uncSF & Starfinder-estimated uncertainty of Flux (mJy) & ... \\
\hline 11 & sky & Starfinder-determined sky brightness (MJy sr ${ }^{-1}$ ) & ... \\
\hline 12 & fwhm & Full-width-at-half-maximum (arcsec) (see Section 7.3) & 0 \\
\hline 13 & flagConf & Confusion flag (see Section 7). 1 for confused; 0 for not confused & .. \\
\hline 14 & corr & Starfinder correlation parameter (see Section 7) & $\cdots$ \\
\hline 15 & epochs & Number of single-epoch images the source was identified in & .. \\
\hline 16 & p100 & Number of PACS $100 \mu \mathrm{m}$ Full List sources within $8 . \prime 8$ & $\ldots$ \\
\hline 17 & s 250 & Number of SPIRE $250 \mu \mathrm{m}$ Full List sources within $12^{\prime \prime} 8$ & $\cdots$ \\
\hline 18 & $\mathrm{~m} 70$ & Number of MIPS $70 \mu \mathrm{m}$ Catalog sources within 12.6 & .. \\
\hline
\end{tabular}

Table 11

SPIRE $250 \mu \mathrm{m}$ Catalog Contents

\begin{tabular}{|c|c|c|c|}
\hline Column & Name & Description & Null \\
\hline 1 & Source name & The identifying name of the source. A description of source name construction is found above & $\ldots$ \\
\hline 2 & R.A. (J2000) & Right ascension, J2000 (deg) & $\ldots$ \\
\hline 3 & Decl. (J2000) & Declination, J2000 (deg) & $\cdots$ \\
\hline 4 & dRA & Right ascension uncertainty (arcsec) & $\cdots$ \\
\hline 5 & dDecl. & Declination uncertainty (arcsec) & $\cdots$ \\
\hline 6 & flux & Starfinder-extracted flux (mJy) & $\cdots$ \\
\hline 7 & unc & Uncertainty of flux (mJy) & ... \\
\hline 8 & flagFlux & Flag describing the quality of the flux measurement (see Section 7.3) & ... \\
\hline 9 & flagStripe & Flag notes if source is in masked region and may suffer from striping. 1 for masked; 0 for unmasked & $\ldots$ \\
\hline 10 & uncSF & Starfinder-estimated uncertainty of Flux (mJy) & .. \\
\hline 11 & sky & Starfinder-determined sky brightness $\left(\mathrm{MJy} \mathrm{sr}^{-1}\right)$ & $\cdots$ \\
\hline 12 & fwhm & Full-width-at-half-maximum ( $\operatorname{arcsec})$ (see Section 7.3) & 0 \\
\hline 13 & flagConf & Confusion flag (see Section 7). 1 for confused; 0 for not confused & $\cdots$ \\
\hline 14 & corr & Starfinder correlation parameter (see Section 7) & $\cdots$ \\
\hline 15 & epochs & Number of single-epoch images the source was identified in & $\cdots$ \\
\hline 16 & p160 & Number of PACS $160 \mu \mathrm{m}$ Full List sources within 12 ".8 & $\cdots$ \\
\hline 17 & s 350 & Number of SPIRE $350 \mu \mathrm{m}$ Full List sources within 18.77 & $\cdots$ \\
\hline 18 & $\mathrm{~m} 70$ & Number of MIPS $70 \mu \mathrm{m}$ Catalog sources within 12 ".8 & $\cdots$ \\
\hline
\end{tabular}

Table 12

SPIRE $350 \mu \mathrm{m}$ Catalog Contents

\begin{tabular}{|c|c|c|c|}
\hline Column & Name & Description & Null \\
\hline 1 & Source name & The identifying name of the source. A description of source name construction is found above & $\ldots$ \\
\hline 2 & R.A. (J2000) & Right ascension, J2000 (deg) & $\ldots$ \\
\hline 3 & Decl. (J2000) & Declination, J2000 (deg) & $\ldots$ \\
\hline 4 & dRA & Right ascension uncertainty ( $\operatorname{arcsec})$ & $\ldots$ \\
\hline 5 & dDecl. & Declination uncertainty (arcsec) & $\cdots$ \\
\hline 6 & flux & Starfinder-extracted flux (mJy) & $\ldots$ \\
\hline 7 & unc & Uncertainty of flux (mJy) & $\ldots$ \\
\hline 8 & flagFlux & Flag describing the quality of the flux measurement (see Section 7.3) & $\ldots$ \\
\hline 9 & flagStripe & Flag notes if source is in masked region and may suffer from striping. 1 for masked; 0 for unmasked & $\ldots$ \\
\hline 10 & uncSF & Starfinder-estimated uncertainty of Flux (mJy) & $\ldots$ \\
\hline 11 & sky & Starfinder-determined sky brightness (MJy sr ${ }^{-1}$ ) & $\ldots$ \\
\hline 12 & fwhm & Full-width-at-half-maximum (arcsec) (see Section 7.3) & 0 \\
\hline 13 & flagConf & Confusion flag (see Section 7). 1 for confused; 0 for not confused & $\ldots$ \\
\hline 14 & corr & Starfinder correlation parameter (see Section 7) & $\ldots$ \\
\hline 15 & epochs & Number of single-epoch images the source was identified in & $\ldots$ \\
\hline 16 & s250 & Number of SPIRE $250 \mu \mathrm{m}$ Full List sources within $18^{\prime \prime} .7$ & .. \\
\hline 17 & s500 & Number of SPIRE $500 \mu \mathrm{m}$ Full List sources within $28^{\prime \prime} .4 / 27^{\prime \prime} 8$ in the LMC/SMC & $\ldots$ \\
\hline 18 & $\mathrm{~m} 70$ & Number of MIPS $70 \mu \mathrm{m}$ Catalog sources within $18^{\prime \prime} 7$ & $\ldots$ \\
\hline
\end{tabular}


Table 13

SPIRE $500 \mu \mathrm{m}$ Catalog Contents

\begin{tabular}{|c|c|c|c|}
\hline Column & Name & Description & Null \\
\hline 1 & Source name & The identifying name of the source. A description of source name construction is found above & $\ldots$ \\
\hline 2 & R.A. (J2000) & Right ascension, J2000 (deg) & $\ldots$ \\
\hline 3 & Decl. (J2000) & Declination, J2000 (deg) & $\ldots$ \\
\hline 4 & dRA & Right ascension uncertainty ( $\operatorname{arcsec})$ & $\ldots$ \\
\hline 5 & dDecl. & Declination uncertainty (arcsec) & $\ldots$ \\
\hline 6 & flux & Starfinder-extracted flux (mJy) & $\ldots$ \\
\hline 7 & unc & Uncertainty of flux (mJy) & $\ldots$ \\
\hline 8 & flagFlux & Flag describing the quality of the flux measurement (see Section 7.3) & $\ldots$ \\
\hline 9 & flagStripe & Flag notes if source is in masked region and may suffer from striping. 1 for masked; 0 for unmasked & $\ldots$ \\
\hline 10 & uncSF & Starfinder-estimated uncertainty of Flux (mJy) & $\ldots$ \\
\hline 11 & sky & Starfinder-determined sky brightness (MJy sr ${ }^{-1}$ ) & $\ldots$ \\
\hline 12 & fwhm & Full-width-at-half-maximum (arcsec) (see Section 7.3) & 0 \\
\hline 13 & flagConf & Confusion flag (see Section 7). 1 for confused; 0 for not confused & $\ldots$ \\
\hline 14 & corr & Starfinder correlation parameter (see Section 7) & $\ldots$ \\
\hline 15 & epochs & Number of single-epoch images the source was identified in & $\ldots$ \\
\hline 16 & s350 & Number of SPIRE $350 \mu \mathrm{m}$ Full List sources within $28^{\prime \prime} .4 / 27^{\prime \prime} 8$ in the LMC/SMC & $\ldots$ \\
\hline 17 & $\mathrm{~m} 160$ & Number of MIPS $160 \mu \mathrm{m}$ Catalog sources within $28^{\prime \prime} .4 / 27^{\prime \prime} .8$ in the LMC/SMC & $\ldots$ \\
\hline
\end{tabular}

Herschel Space Observatory, HSO; II specifies the instrument (P for PACS, S for SPIRE) and the wavelength (2, 3, 1, 2, 3 for $100,160,250,350$, and $500 \mu \mathrm{m}$, respectively); PPPP is a four-character code for the HERITAGE project, HERI; E denotes the epoch (all sources are marked with $\mathrm{C}$, combinedepoch detections), and $\mathrm{L}$ designates the list type, $\mathrm{C}$ for Catalog. RR.RRRR and DD.DDD are the right ascension and declination, respectively, in degrees to four decimal places, and $\mathrm{S}$ is the sign of the declination.

\section{REFERENCES}

Aguirre, J. E., Bezaire, J. J., Cheng, E. S., et al. 2003, ApJ, 596, 273 Alcock, C., Allsman, R. A., Alves, D. R., et al. 2000, ApJ, 542, 281 Aniano, G., Draine, B. T., Gordon, K. D., \& Sandstrom, K. 2011, PASP, 123,1218

Asplund, M., Grevesse, N., Sauval, A. J., Allende Prieto, C., \& Kiselman, D. 2004, A\&A, 417, 751

Bekki, K., \& Chiba, M. 2005, MNRAS, 356, 680

Bennett, C. L., Bay, M., Halpern, M., et al. 2003a, ApJ, 583, 1

Bennett, C. L., Hill, R. S., Hinshaw, G., et al. 2003b, ApJS, 148, 97

Bernard, J.-P., Reach, W. T., Paradis, D., et al. 2008, AJ, 136, 919

Bertin, E., \& Arnouts, S. 1996, A\&AS, 117, 393

Besla, G., Kallivayalil, N., Hernquist, L., et al. 2007, ApJ, 668, 949

Boggess, N. W., Mather, J. C., Weiss, R., et al. 1992, ApJ, 397, 420

Bolatto, A. D., Leroy, A. K., Jameson, K., et al. 2011, ApJ, 741, 12

Bolatto, A. D., Simon, J. D., Stanimirović, S., et al. 2007, ApJ, 655, 212

Bot, C., Boulanger, F., Lagache, G., Cambrèsy, L., \& Egret, D. 2004, A\&A, 423, 567

Boyer, M. L., Sargent, B., van Loon, J. T., et al. 2010, A\&A, 518, L142

Boyer, M. L., Srinivasan, S., Riebel, D., et al. 2012, ApJ, 748, 40

Boyer, M. L., Srinivasan, S., van Loon, J. T., et al. 2011, AJ, 142, 103

Cantalupo, C. M., Borrill, J. D., Jaffe, A. H., Kisner, T. S., \& Stompor, R. 2010, ApJS, 187, 212

Carlson, L. R., Sewiło, M., Meixner, M., Romita, K. A., \& Lawton, B. 2012, A\&A, 542, A66

Chen, C.-H. R., Indebetouw, R., Muller, E., et al. 2013, ApJ, submitted

Cioni, M.-R. L., Clementini, G., Girardi, L., et al. 2011, A\&A, 527, A116

Clayton, G. C., De Marco, O., Whitney, B. A., et al. 2011, AJ, 142, 54

Clayton, G. C., Sargent, B., Boyer, M. L., et al. 2010, ApJ, 722, 1131

Cohen, R. S., Dame, T. M., Garay, G., Montani, J., \& Rubio, M. 1988, ApJL, 331, L95

Diolaiti, E., Bendinelli, O., Bonaccini, D., et al. 2000, A\&AS, 147, 335

Dobashi, K., Bernard, J.-P., Hughes, A., et al. 2008, A\&A, 484, 205

Egan, M. P., Van Dyk, S. D., \& Price, S. D. 2001, AJ, 122, 1844

Fixsen, D. J., Cheng, E. S., Cottingham, D. A., et al. 1994, ApJ, 420, 457

Fritz, J., Gentile, G., Smith, M., et al. 2012, A\&A, 546, A34

Fukui, Y., Kawamura, A., Minamidani, T., et al. 2008, ApJS, 178, 56

Galliano, F., Hony, S., Bernard, J.-P., et al. 2011, A\&A, 536, A88
Gardiner, L. T., \& Noguchi, M. 1996, MNRAS, 278, 191

Gaustad, J. E., McCullough, P. R., Rosing, W., \& Van Buren, D. 2001, PASP, 113,1326

Gordon, K. D., Bot, C., Muller, E., et al. 2009, ApJL, 690, L76

Gordon, K. D., Clayton, G. C., Misselt, K. A., Landolt, A. U., \& Wolff, M. J. 2003, ApJ, 594, 279

Gordon, K. D., Galliano, F., Hony, S., et al. 2010, A\&A, 518, L89

Gordon, K. D., Meixner, M., Meade, M. R., et al. 2011, AJ, 142, 102

Griffin, M. J., Abergel, A., Abreu, A., et al. 2010, A\&A, 518, L3

Grondin, L., Demers, S., \& Kunkel, W. E. 1992, AJ, 103, 1234

Gruendl, R. A., \& Chu, Y.-H. 2009, ApJS, 184, 172

Hambly, N. C., Dufton, P. L., Keenan, F. P., et al. 1994, A\&A, 285, 716

Harris, J. 2007, ApJ, 658, 345

Harris, J., \& Zaritsky, D. 2004, AJ, 127, 1531

Harris, J., \& Zaritsky, D. 2009, AJ, 138, 1243

Hindman, J. V., Kerr, F. J., \& McGee, R. X. 1963, AuJPh, 16, 570

Hony, S., Galliano, F., Madden, S. C., et al. 2010, A\&A, 518, L76

Israel, F. P., Wall, W. F., Raban, D., et al. 2010, A\&A, 519, A67

Ita, Y., Tanabé, T., Matsunaga, N., et al. 2004, MNRAS, 347, 720

Kawamura, A., Mizuno, Y., Minamidani, T., et al. 2009, ApJS, 184, 1

Kemper, F., Woods, P. M., Antoniou, V., et al. 2010, PASP, 122, 683

Kennicutt, R. C., Armus, L., Bendo, G., et al. 2003, PASP, 115, 928

Kennicutt, R. C., Calzetti, D., Aniano, G., et al. 2011, PASP, 123, 1347

Kim, S., Kwon, E., Madden, S. C., et al. 2010, A\&A, 518, L75

Kim, S., Staveley-Smith, L., Dopita, M. A., et al. 1998, ApJ, 503, 674

Kim, S., Staveley-Smith, L., Dopita, M. A., et al. 2003, ApJS, 148, 473

Lee, J.-K., Rolleston, W. R. J., Dufton, P. L., \& Ryans, R. S. I. 2005, A\&A, 429, 1025

Leroy, A., Bolatto, A., Stanimirović, S., et al. 2007, ApJ, 658, 1027

Madau, P., Ferguson, H. C., Dickinson, M. E., et al. 1996, MNRAS, 283, 1388

Maíz Apellániz, J., \& Rubio, M. 2012, A\&A, 541, A54

Mathewson, D. S. 1985, PASAu, 6, 104

Matsuura, M., Dwek, E., Meixner, M., et al. 2011, Sci, 333, 1258

Meixner, M., Galliano, F., Hony, S., et al. 2010, A\&A, 518, L71

Meixner, M., Gordon, K., Indebetouw, R., et al. 2006, AJ, 132, 2268

Mizuno, N., Rubio, M., Mizuno, A., et al. 2001, PASJ, 53, L45

Molinari, S., Schisano, E., Faustini, F., et al. 2011, A\&A, 530, A133

Molinari, S., Swinyard, B., Bally, J., et al. 2010, PASP, 122, 314

Muller, E., Staveley-Smith, L., Zealey, W., \& Stanimirović, S. 2003a, MNRAS, 339,105

Muller, E., Staveley-Smith, L., \& Zealey, W. J. 2003b, MNRAS, 338, 609

Ngeow, C., \& Kanbur, S. M. 2008, ApJ, 679, 76

Nikolaev, S., \& Weinberg, M. D. 2000, ApJ, 542, 804

Otsuka, M., van Loon, J. T., Long, K. S., et al. 2010, A\&A, 518, L139

Ott, S. 2010, in ASP Conf. Ser. 434, Astronomical Data Analysis Software and Systems XIX, ed. Y. Mizumoto, K.-I. Morita, \& M. Ohishi (San Francisco, CA: ASP), 139

Paladini, R., Linz, H., Altrieri, B., \& Ali, B. 2012, PACS Herschel Observatory Report, PICC-NHSC-TR-034, Version 1.0

Pei, Y. C., Fall, S. M., \& Hauser, M. G. 1999, ApJ, 522, 604

Pilbratt, G. L., Riedinger, J. R., Passvogel, T., et al. 2010, A\&A, 518, L1 
Planck Collaboration, Ade, P. A. R., Aghanim, N., et al. 2011, A\&A, 536, A17 Poglitsch, A., Waelkens, C., Geis, N., et al. 2010, A\&A, 518, L2

Reid, M. J., Menten, K. M., Zheng, X. W., Brunthaler, A., \& Xu, Y. 2009, ApJ, 705,1548

Riebel, D., Srinivasan, S., Sargent, B., \& Meixner, M. 2012, ApJ, 753, 71

Roman-Duval, J., Israel, F. P., Bolatto, A., et al. 2010, A\&A, 518, L74

Roussel, H. 2012, PASP, submitted (arXiv:1205.2576)

Rubio, M., Garay, G., Montani, J., \& Thaddeus, P. 1991, ApJ, 368, 173

Russell, S. C., \& Dopita, M. A. 1992, ApJ, 384, 508

Sauvage, M., \& Okumura, K. 2009, PACS Herschel Technical Report, Document SAp-PACS-MS-0712-09

Schwering, P. B. W. 1989, A\&AS, 79, 105

Schwering, P. B. W., \& Israel, F. P. 1989, A\&AS, 79, 79

Sewiło, M., Carlson, L. R., Seale, J. P., et al. 2013, ApJ, submitted

Sewiło, M., Indebetouw, R., Carlson, L. R., et al. 2010, A\&A, 518, L73

Silverberg, R. F., Hauser, M. G., Boggess, N. W., et al. 1993, Proc. SPIE, 2019, 180

Skibba, R., Engelbracht, C. W., Babler, B., et al. 2012, ApJ, 761, 42

Skrutskie, M. F., Cutri, R. M., Stiening, R., et al. 2006, AJ, 131, 1163

Srinivasan, S., Meixner, M., Leitherer, C., et al. 2009, AJ, 137, 4810

Stanimirović, S., Staveley-Smith, L., Dickey, J. M., Sault, R. J., \& Snowden, S. L. 1999, MNRAS, 302, 417
Staveley-Smith, L., Juraszek, S., Koribalski, B. S., et al. 1998, AJ, 116, 2717 Staveley-Smith, L., Kim, S., Calabretta, M. R., Haynes, R. F., \& Kesteven, M. J. 2003, MNRAS, 339, 87

Subramanian, S., \& Subramaniam, A. 2009, A\&A, 496, 399

Subramanian, S., \& Subramaniam, A. 2010, A\&A, 520, A24

Subramanian, S., \& Subramaniam, A. 2012, ApJ, 744, 128

Szewczyk, O., Pietrzyński, G., Gieren, W., et al. 2009, AJ, 138, 1661

Tody, D. 1993, in ASP Conf. Ser. 52, Astronomical Data Analysis Software and Systems II, ed. R. J. Hanisch, R. J. V. Brissenden, \& J. Barnes (San Francisco, CA: ASP), 173

Tumlinson, J., Shull, J. M., Rachford, B. L., et al. 2002, ApJ, 566, 857

Udalski, A., Soszynski, I., Szymanski, M. K., et al. 2008a, AcA, 58, 89

Udalski, A., Soszyński, I., Szymański, M. K., et al. 2008b, AcA, 58, 329

Verdugo, C., Rubio, M., Bolatto, A., et al. 2013, A\&A, submitted

Whitney, B. A., Sewilo, M., Indebetouw, R., et al. 2008, AJ, 136, 18

Wilke, K., Klaas, U., Lemke, D., et al. 2004, A\&A, 414, 69

Wong, T., Hughes, A., Ott, J., et al. 2011, ApJS, 197, 16

Wright, E. L., Mather, J. C., Bennett, C. L., et al. 1991, ApJ, 381, 200

Zaritsky, D., \& Harris, J. 2004, ApJ, 604, 167

Zaritsky, D., Harris, J., Thompson, I. B., \& Grebel, E. K. 2004, AJ, 128, 1606

Zaritsky, D., Harris, J., Thompson, I. B., Grebel, E. K., \& Massey, P. 2002, AJ, 123,855 


\title{
ERRATUM: "THE HERSCHEL INVENTORY OF THE AGENTS OF GALAXY EVOLUTION (HERITAGE) IN THE MAGELLANIC CLOUDS, A HERSCHEL OPEN TIME KEY PROGRAM” (2013, AJ, 146, 62)
}

\author{
M. Meixner ${ }^{1,2}$, P. Panuzzo ${ }^{3,4}$, J. Roman-Duval ${ }^{1}$, C. Engelbracht ${ }^{5,6}$, B. Babler ${ }^{7}$, J. Seale ${ }^{1}$, S. Hony $^{3}$, E. Montiel ${ }^{5,8}$, \\ M. SAuvage ${ }^{3}$, K. Gordon ${ }^{1}$, K. Misselt ${ }^{5}$, K. OKumura ${ }^{3}$, P. Chanial ${ }^{3}$, T. Beck ${ }^{1}$, J.-P. Bernard ${ }^{9,10}$, A. Bolatto $^{11}$, C. Bot ${ }^{12,13}$, \\ M. L. Boyer ${ }^{1,14,15}$, L. R. CARlson ${ }^{16}$, G. C. Clayton $^{8}$, C.-H. R. Chen ${ }^{17}$, D. Cormier ${ }^{3}$, Y. Fukui ${ }^{18}$, M. Galametz ${ }^{19}$, \\ F. Galliano ${ }^{3}$, J. L. Hora ${ }^{20}$, A. Hughes ${ }^{21}$, R. Indebetouw ${ }^{22}$, F. P. Israel ${ }^{16}$, A. Kawamura ${ }^{23}$, F. KemPer ${ }^{24}$, S. Kim ${ }^{25}$, E. KwON ${ }^{25}$, \\ V. Lebouteiller ${ }^{3}$, A. Li $^{26}$, K. S. Long ${ }^{1}$, S. C. Madden ${ }^{3}$, M. Matsuura ${ }^{27}$, E. Muller ${ }^{23}$, J. M. Oliveira ${ }^{28}$, T. Onishi ${ }^{29}$, \\ M. Otsuka ${ }^{24}$, D. Paradis ${ }^{9,10}$, A. Poglitsch ${ }^{30}$, W. T. Reach ${ }^{31}$, T. P. Robitaille ${ }^{21}$, M. Rubio ${ }^{32}$, B. SARgent ${ }^{1,33}$, M. SeWiŁo ${ }^{2}$, \\ R. Skibba ${ }^{5}$, L. J. Smith $^{34}$, S. Srinivasan ${ }^{35,24}$, A. G. G. M. Tielens ${ }^{16}$, J. Th. van Loon ${ }^{28}$, And B. Whitney ${ }^{7}$ \\ ${ }^{1}$ Space Telescope Science Institute, 3700 San Martin Drive, Baltimore, MD 21218, USA; meixner@stsci.edu \\ 2 The Johns Hopkins University, Department of Physics and Astronomy, 366 Bloomberg Center, 3400 N. Charles Street, Baltimore, MD 21218, USA \\ ${ }^{3}$ CEA, Laboratoire AIM, Irfu/SAp, Orme des Merisiers, F-91191 Gif-sur-Yvette, France \\ ${ }^{4}$ CNRS, Observatoire de Paris - Lab. GEPI, Bat. 11, 5, place Jules Janssen, F-92195 Meudon CEDEX, FRANCE, France \\ ${ }^{5}$ Steward Observatory, University of Arizona, 933 North Cherry Ave., Tucson, AZ 85721, USA \\ ${ }^{6}$ Raytheon Company, 1151 East Hermans Road, Tucson, AZ 85756, USA \\ ${ }^{7}$ Department of Astronomy, 475 North Charter St., University of Wisconsin, Madison, WI 53706, USA \\ ${ }^{8}$ Louisiana State University, Department of Physics \& Astronomy, 233-A Nicholson Hall, Tower Dr., Baton Rouge, LA 70803-4001, USA \\ CNRS, IRAP, 9 Av. colonel Roche, BP 44346, F-31028 Toulouse Cedex 4, France \\ ${ }^{10}$ Universit de Toulouse, UPS-OMP, IRAP, 31028 Toulouse Cedex 4, France \\ ${ }^{11}$ Department of Astronomy, Lab for Millimeter-wave Astronomy, University of Maryland, College Park, MD 20742-2421, USA \\ ${ }^{12}$ Université de Strasbourg, Observatoire Astronomique de Strasbourg, 11, rue de l'universite, F-67000 Strasbourg, France \\ ${ }^{13}$ CNRS, Observatoire Astronomique de Strasbourg, UMR7550, F-67000 Strasbourg, France \\ ${ }^{14}$ Observational Cosmology Lab, Code 665, NASA Goddard Space Flight Center, Greenbelt, MD 20771, USA \\ ${ }^{15}$ Oak Ridge Associated Universities (ORAU), Oak Ridge, TN 37831, USA \\ ${ }^{16}$ Sterrewacht Leiden, Leiden University, P.O. Box 9513, NL-2300 RA Leiden, The Netherlands \\ ${ }_{17}^{17}$ Max-Planck-Institut für Radioastronomie Auf dem Hügel 69, 53121 Bonn, Germany \\ ${ }^{18}$ Department of Astrophysics, Nagoya University, Chikusa-ku, Nagoya 464-8602, Japan \\ ${ }^{19}$ Institute of Astronomy, University of Cambridge, Madingley Road, Cambridge CB3 0HA, UK \\ ${ }^{20}$ Center for Astrophysics, 60 Garden St., MS 67, Harvard University, Cambridge, MA 02138, USA \\ ${ }^{21}$ Max-Planck-Institut für Astronomie, Königstuhl 17, D-69117 Heidelberg, Germany \\ ${ }^{22}$ Department of Astronomy, University of Virginia, and National Radio Astronomy Observatory, 520 Edgemont Road, Charlottesville, VA 22903, USA \\ ${ }_{23}$ National Astronomical Observatory of Japan, 2-21-1, Osawa, Mitaka, Tokyo, 181-8588, Japan \\ ${ }^{24}$ Academia Sinica, Institute of Astronomy and Astrophysics, PO Box 23-141, Taipei 10617, Taiwan \\ ${ }^{25}$ Department of Astronomy and Space Science, Sejong University, 98 Gunja-dong, Gwangjin-gu, 143-747, Seoul, Korea \\ 26314 Physics Building, Department of Physics and Astronomy, University of Missouri, Columbia, MO 65211, USA \\ ${ }^{27}$ Department of Physics and Astronomy, University College London, Gower Street, London WC1E 6BT, UK \\ ${ }^{28}$ School of Physical \& Geographical Sciences, Lennard-Jones Laboratories, Keele University, Staffordshire ST5 5BG, UK \\ ${ }^{29}$ Department of Physical Science, Osaka Prefecture University, Gakuen 1-1, Sakai, Osaka 599-8531, Japan \\ ${ }^{30}$ Max-Planck-Institut für extraterrestrische Physik, Giessenbachstraße D-85748 Garching, Germany \\ ${ }^{31}$ Stratospheric Observatory for Infrared Astronomy, Universities Space Research Association, Mail Stop 232-11, Moffett Field, CA 94035, USA \\ ${ }^{32}$ Departamento de Astronomía, Universidad de Chile, Casilla 36-D, Santiago, Chile \\ ${ }^{33}$ Center for Imaging Science and Laboratory for Multiwavelength Astrophysics, Rochester Institute of Technology, \\ 54 Lomb Memorial Drive, Rochester, NY 14623, USA \\ ${ }^{34}$ Space Telescope Science Institute and European Space Agency, 3700 San Martin Drive, Baltimore, MD 21218, USA \\ ${ }^{35}$ UPMC-CNRS UMR7095, Institute d'Astrophysique de Paris, F-75014 Paris, France \\ Received 2014 December 17; accepted 2014 December 26; published 2015 February 2
}

We provide a corrected Table 4 that lists the total galaxy fluxes for the HERTIAGE bands and corresponding Figure 19 which plots these fluxes in comparison to prior measurements These corrected fluxes differ by up to $41 \%$ to what we reported in the original paper. These revised fluxes utilize a more appropriate subtraction of the Milky Way Foreground Cirrus emission which contaminates especially the PACS 100 and $160 \mu \mathrm{m}$ bands. The subtraction process uses the HI $21 \mathrm{~cm}$ emission to develop a model for the MW cirrus dust emission. In addition, the better subtraction process corrected for an over subtraction of the background in the SPIRE images of the SMC that occured during the original data processing. The need for these better foreground subtractions was realized while working on an analysis of the dust masses and gas-to-dust ratios in the LMC and SMC reported by Gordon et al. (2014) and Roman-Duval et al. (2014). After the subtraction has been done, the fluxes were derived by simply summing up all the pixels in the image. The errors we quote for the fluxes reflect the absolute flux calibration errors for extended sources which are $~ 10 \%$ for PACS and $\sim 8 \%$ for SPIRE. In the revised Figure 19, we confirm that these corrected global fluxes remain within the range of prior global measurements for both the LMC and SMC. Indeed, the shape of the corrected spectral energy distributions appears better aligned with prior measurements. 

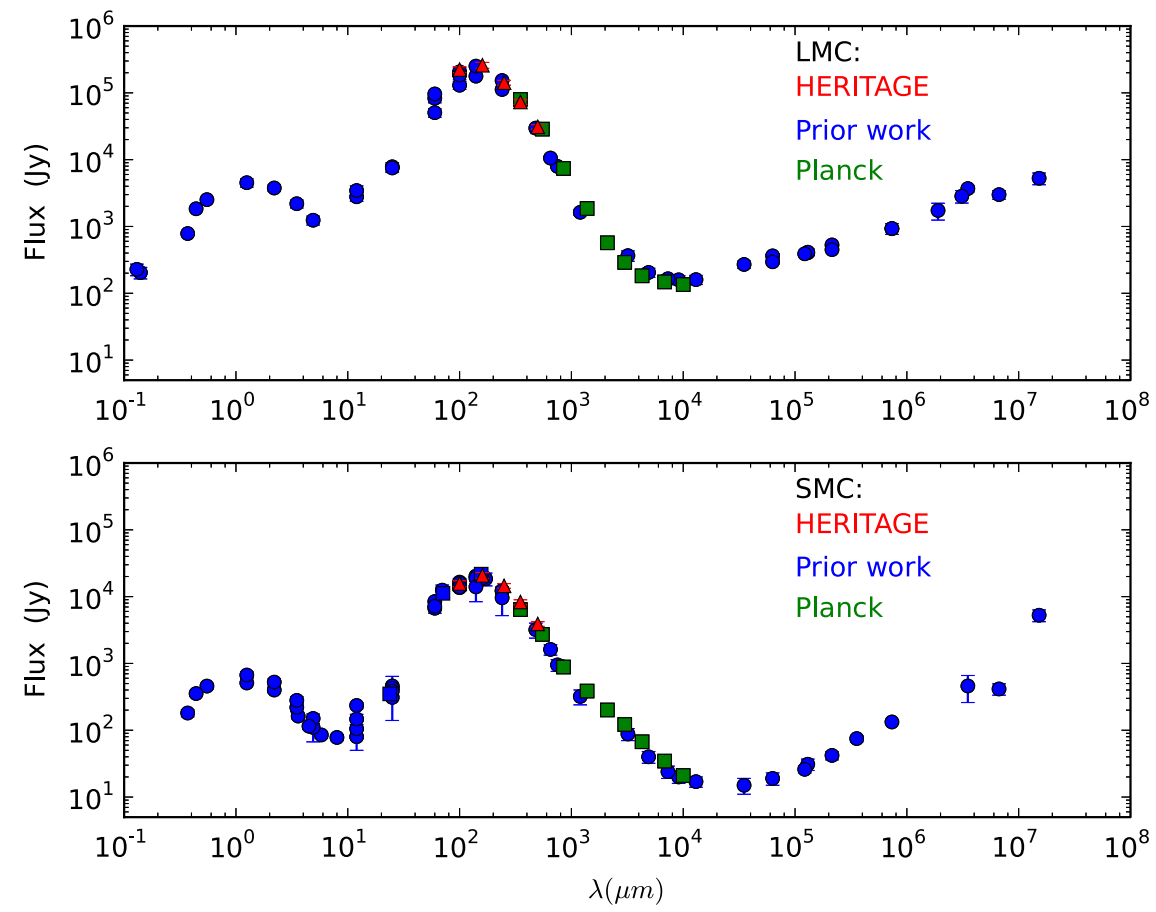

Figure 19. The spectral energy distributions of the whole LMC and SMC cover the UV to radio wavelengths. Prior work summarized by Israel et al. (2010) includes data from ground and space based missions such as IRAS, DIRBE, TopHat, and WMAP. For the SMC, we also include prior work (blue squares) from Gordon et al. (2011). The HERITAGE photometry measured in the PACS and SPIRE bands listed in Table 4 are shown in red. The Planck measurements are shown in green (Planck collaboration 2011).

Table 4

Total Galaxy Fluxes in SED

\begin{tabular}{|c|c|c|c|c|c|}
\hline \multirow[b]{2}{*}{ Parameter } & \multicolumn{2}{|c|}{ PACS $\lambda(\mu \mathrm{m})$} & \multicolumn{3}{|c|}{$\operatorname{SPIRE} \lambda(\mu \mathrm{m})$} \\
\hline & 100 & 160 & 250 & 350 & 500 \\
\hline LMC flux (Jy) & $2.2 \pm 0.2 \times 10^{5}$ & $2.6 \pm 0.3 \times 10^{5}$ & $1.4 \pm 0.1 \times 10^{5}$ & $7.3 \pm 0.6 \times 10^{4}$ & $3.1 \pm 0.3 \times 10^{4}$ \\
\hline SMC flux (Jy) & $1.6 \pm 0.2 \times 10^{4}$ & $2.1 \pm 0.2 \times 10^{4}$ & $1.5 \pm 0.1 \times 10^{4}$ & $8.3 \pm 0.7 \times 10^{3}$ & $3.9 \pm 0.3 \times 10^{3}$ \\
\hline
\end{tabular}

\section{REFERENCES}

Gordon, K. D., Meixner, M., Meade, M. R., et al. 2011, AJ, 142, 102 Gordon, K. D., Roman-Duval, J., Bot, C., et al. 2014, ApJ, 797, 85
Israel, F. P., Wall, W. F., Raban, D., et al. 2010, A\&A, 519, A67

Planck Collaboration, Ade, P. A. R.., Aghanim, N., et al. 2011, A\&A, 536, A17

Roman-Duval, J., Gordon, K. D., Meixner, M., et al. 2014, ApJ, 797, 86 